SSCL-Preprint-503

CTP-TAMU-52/93

NUB-TH-3073-93

\title{
SUPERSYMMETRY AND SUPERGRAVITY: PHENOMENOLOGY AND GRAND UNIFICATION
}

\author{
R. ARNOWITT ${ }^{* a b}$ and PRAN NATH ${ }^{c}$ \\ ta Center for Theoretical Physics, Department of Physics \\ Texas A\&M University, College Station, TX 77843-4242 \\ 'Physics Research Division, Superconducting Super \\ Collider Laboratory, Dallas, TX 75237 \\ cDepartment of Physics, Northeastern University \\ Boston, MA 02115
}

\begin{abstract}
A survey is given of supersymmetry and supergravity and their phenomenology. Some of the topics discussed are the basic ideas of global supersymmetry, the minimal supersymmetric Standard Model (MSSM) and its phenomenology, the basic ideas of local supersymmetry (supergravity), grand unification, supersymmetry breaking in supergxavity grand unified models, radiative breaking of $S U(2) \times U(1)$, proton decay, cosmological constraints, and predictions of supergravity grand unified models. While the number of detailed derivations are necessarily limited, a sufficient number of results are given so that a reader can get a working knowledge of this field.
\end{abstract}

\section{Introduction}

The Standard Model (SM) with three generations of quarks and leptons, based on the gauge group $S U(3) \times S U(2) \times U(1)$ and possessing one Higgs doublet to realize electroweak breaking, is in excellent agreement with all current data. In fact, the Standard Model is one of the most successful theories ever constructed, and at present accounts for all microscopic physics. In spite of this, there is a general belief that the theory carries within it the seeds of its own destruction, and should be expected to break down in the TeV energy domain. A number of suggestions have been made to generalize the ideas of the SM to circumvent its problems but yet reduce to it at energies $\lesssim 100 \mathrm{GeV}$ (and hence maintain its successes). These include supersymmetry models, technicolor models, and models based on the existance of a $t \bar{t}$ condensate. Each of these are different ways of treating the quadratic divergence of the Higgs boson self energy.

The simplest technicolor models appear to be inconsistent with current LEP data [1], though more complicated models may be able to evade this problem. The simplest models of $t \bar{t}$ condensates favor a rather heavy $t$-quark $\left(m_{t} \gtrsim 200 \mathrm{Gev}\right)$ and a heavy Higgs boson $\left(m_{H} \gtrsim 300 \mathrm{GeV}\right)$ which, while not excluded, is not favored by current LEP data [2]. Again more complicated models may be able to modify

\footnotetext{
-Lectures at VII J. A. Swieca Summer School, Campos do Jordao, Brazil, 1993.
}

tPermanent Addreas 
these predictions. On the other hand, there has been the first indication of supersymmetric grand unification arising from the precision measurement at LEP and elsewhere of the SM coupling constants $\alpha_{1}\left(M_{Z}\right), \alpha_{2}\left(M_{Z}\right), \alpha_{3}\left(M_{Z}\right)$ at scale $Q=M_{Z}$. Using the renormalization group equations, grand unification does not occur for the SM, but does appear to take place for its supersymmetrized version at a Gut scale of [3] $M_{G} \simeq 10^{16} \mathrm{GeV}$. This may, of course, be just a numerical accident, but if one takes this result seriously, it suggests the validity of the combined ideas of supersymmetry and grand unification. Further, in order to combine supersymmetry and grand unification, in a phenomenologically viable way, one needs to make use of local supersymmetry i.e. supergravity. It thus appears to be of interest to ask what other phenomena can one use to test the validity of supergravity grand unified models.

In these talks, we will survey various aspects of supersymmetry and supergravity Gut models. While it will not be possible to give here the derivation of many of the basic results, the talks will be pedagogic in the sense that enough will be included to give a working knowledge of this area of particle physics, as well as some of the recent results. While supergravity may be viewed to arise as a consequence of string theory, we won't impose any particular string assumptions on the analysis. The natural scale for superstring theory is the Planck scale

$$
M_{P \ell}=\left(8 \pi G_{N}\right)^{-1 / 2}=2.4 \times 10^{18} \mathrm{GeV}
$$

Since $M_{G} / M_{P \ell} \approx 10^{-2}$ it may be that string effects are small corrections to supergravity Gut models, and that such models are thus reasonably self contained and represent another "way station" on the road to a more fundamental theory.

\section{What's Wrong with the Standard Model?}

The Standard Model (SM) with three generations of quarks and leptons is based on the gauge group $S U(3)_{C} \times S U(2)_{L} \times U(1)_{Y}$ where $C=$ color, $L=$ left and $Y=$ hypercharge. Quarks and leptons are left-handed doublets and right-handed singlets:

$$
q_{i}^{\alpha}=\left(u_{i}, d_{i}\right)_{L} ; u_{i R}, d_{i R} ; l_{i}^{\alpha}=\left(\nu_{i}, e_{i}\right)_{L} ; e_{i R}
$$

where $i=1,2,3$ is the generation index and $\alpha=1,2$ is the $S U(2)_{L}$ doublet index. In addition there is a Higgs doublet:

$$
H^{\alpha}=\left(H^{(+)}, H^{(0)}\right) ; H_{\alpha} \equiv \varepsilon_{\alpha \beta} H^{\beta}
$$

with $\varepsilon_{\alpha \beta}=-\varepsilon_{\beta \alpha}$ and $\varepsilon_{12}=+1$.

The dynamics of the SM consists of the gauge interactions, the Yukawa coupling and the Higgs potential. The first are constructed in the usual way:

$$
\partial_{\mu} \rightarrow \partial_{\mu}-i\left[g_{3} A_{\mu}^{a} T_{C}^{a}+g_{2} B_{\mu}^{m} T_{L}^{m}+g^{\prime} B_{\mu}(Y / 2)\right.
$$




\section{DISCLAIMER}

Portions of this document may be illegible in electronic image products. Images are produced from the best available original document. 
where $T_{C}^{a}=\left(\lambda^{a} / 2 ; 0\right)$ for (quarks; leptons, Higgs), where the $\lambda^{a}, a=1 \cdots 8$ are GellMann matrices: $T_{L}^{m}=\left(\tau^{m} / 2 ; 0\right)$ for $S U(2)_{L}$ (doublets; singlets), where the $\tau^{m}$ are Pauli matrices; and $Y$ is defined by $Q=T_{L}^{3}+Y / 2$. The $A_{\mu}^{a}(x), B_{\mu}^{m}(x)$ and $B_{\mu}$ are the color gluons, and the $S U(2)_{L}$ and hypercharge gauge bosons, and $g_{3}, g_{2}$ and $g^{\prime}$ are their corresponding coupling constants.

The Yukawa interactions are specified by the potential

$$
V_{Y}=\lambda_{i j}^{(u)} H_{\alpha} \bar{u}_{i R} q_{j}^{\alpha}+\lambda_{i j}^{(d)} H^{\alpha} \bar{q}_{i}^{\alpha} d_{R j}+\lambda_{i j}^{(e)} H^{\alpha} \boldsymbol{l}_{i}^{\alpha} e_{R j}
$$

where $\lambda_{i j}^{(u, d, e)}$ are the Yukawa coupling constant. The Higgs potential is given by

$$
V_{H}=-m^{2} H^{\alpha \dagger} H^{\alpha}+\lambda\left(H^{\alpha \dagger} H^{\alpha}\right)^{2} ; m^{2}, \lambda>0
$$

The Higgs potential gives rise to a spontaneous breaking of $S U(2) \times U(1)$ due to the peculiar sign chosen in the mass term. One finds, inserting $H^{(0)}=\left(v+H^{(0)}\right) / \sqrt{2}$, where $v=\sqrt{2}\left\langle H^{(0)}\right\rangle$, that minimizing $V_{H}$ yields $v=\mathrm{m}^{2} / \sqrt{2} \lambda$ and the Higgs boson $H^{(0)^{\prime}}$ then possesses a positive tree level (mass) ${ }^{2}$ of $m_{H}^{2}=2 m^{2}>0$. The Yukawa interactions then gives rise to masses for the quarks and leptons, and by making unitary transformations on the $R$ and $L$ fields one may go to the mass diagonal states, which for the down quarks are $d_{i}^{\prime}=V_{i j} d_{j}$ where $V$ is the CKM matrix. The current experimental evaluations of $V_{i j}$ are given in Ref. [4]. The gauge interactions also give rise to $W^{ \pm}$and $Z$ boson masses e.g. $M_{W}=g_{2} v / 2$ which implies $v=246 \mathrm{GeV}$. Alternately one may write

$$
M_{W}=\frac{g_{2}}{\sqrt{\lambda}} \frac{m_{H}}{2 \sqrt{2}}
$$

showing that $m_{H}$ sets the electroweak mass scale.

The SM has passed a very large number of experimental tests and is currently in excellent agreement with all the data. However, from the theoretical side, many aspects of the SM are unsatisfactory, leading one to suspect that one is seeing the low energy manisfestation of some more fundamental theory. We list here some of these difficulties:

(i) The SM has 19 adjustable parameters. These are 3 lepton masses $\left(m_{e}, m_{\mu}, m_{\tau}\right)$; 6 quark masses $\left(m_{u_{i}}, m_{d_{i}}, i=1,2,3\right) ; 3$ coupling constants $\alpha_{3}=g_{3}^{2} / 4 \pi, \alpha_{2}=g_{2}^{2} / 4 \pi$, $\alpha_{Y}=g^{2} / 4 \pi ; 4$ parameters in the CKM matrix ( 3 angles, 1 CP violating phase); 2 Higgs potential parameters $\left(\lambda, m^{2}\right)$; and the strong CP violating parameter $\left(\theta_{Q C D} \tilde{F}_{a}^{\mu \nu} F_{\mu \nu}^{a}\right)$. One must go to experiment to fit these [4]. (The current bounds on $m_{t}$ and $m_{H}$ are $m_{t}>118 \mathrm{GeV}$ (CDF) and $m_{H}>62.5 \mathrm{GeV}$ (combined LEP analysis). Clearly a fundamental theory is expected to have fewer arbitrary parameters.

(ii) The breaking of $S U(2) \times U(1)$ is inserted by hand (by choosing $-m^{2}$ in Eq. (6) instead of $+m^{2}$ ), rather than being a consequence of the theoretical principles of the model. Thus while the SM can accommodate spontaneous electroweak breaking, it does not explain its origin. 
(iii) There is no real electroweak unification as the SM group is a product group. Also there is a mysterious symmetry between quarks and leptons: both have $L$ particles (not anti-particles) as $S U(2)$ doublets and both obey the relation $Q=T_{3}+Y / 2$ which relates quark and lepton charges. This suggests the existance of grand unification with $S U(3) \times S U(2) \times U(1)$ embedded in a simple group [e.g. $S U(5)$ or $O(10)]$ with quarks and leptons appearing in the same representation.

(iv) Perhaps the most serious issue is the "gauge hierarchy" problem. The loop corrections to $m_{H}$ (an example is given in Fig. 1) are quadratically divergent leading to a mass

$$
m_{H}^{2}=2 m^{2}+c(\bar{\alpha} / 4 \pi) \wedge^{2}
$$

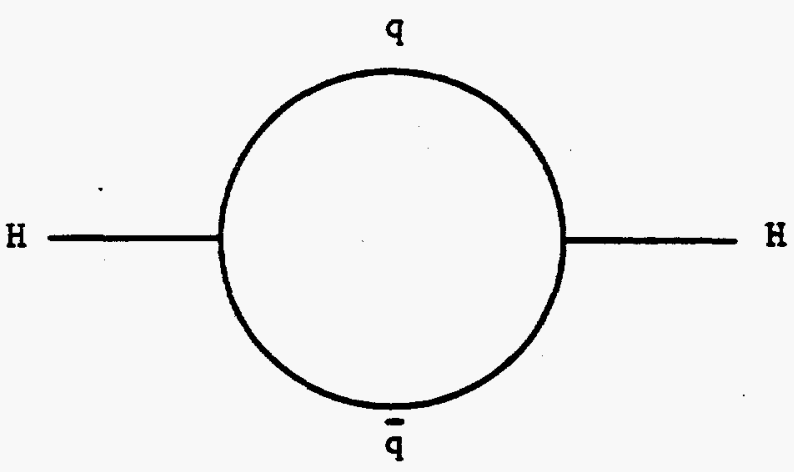

Figure 1: One loop correction to Higgs mass from Higgs couplings to quarks.

where $\tilde{\alpha}$ is a coupling constant, and $\Lambda$ is the cutoff. One may view $\wedge$ as the scale where new physics occurs (which cuts off the quadratic divergence). How high can $\wedge$ be? As $\wedge$ increases, $m_{H}$ (which by Eq. (7) scales electroweak physics) increases, and hence the electroweak scale is driven close to the large scale $\wedge$. This is known as the "gauge hierarchy" problem. An alternate possibility is to choose $m^{2}$ to cancel the large loop correction. This then results in the "fine tuning" problem. For example if $\wedge=M_{G} \sim 10^{15} \mathrm{GeV}$, one must fine tune $m^{2}$ to 24 decimal places (!) and in fact troubles begin already in the TeV region. Thus one expects the SM to break down in the TeV domain, which is why the SSC and LHC are expected to uncover new physics.

\section{Global Supersymmetry}

Supersymmetry attempts to shed light on items (ii), (iii) and (iv) above. It only makes a small amount of progress on item (i), which presumably needs a much deeper theory to approach (such as string theory). We discuss first global supersymmetry. 
Supersymmetry is a symmetry between bosons and fermions, i.e. it requires that the number of bose and fermi helicity states in a multiplet be equal. It was first introduced on purely "aesthetic" grounds, that nature should be even handed between boson and fermions.

One may realize supersymmetry (SUSY) algebraically in the following way [5]. Let $Q_{\alpha}$ be a $L$ spinor obeying the following anti-commutation relations [6]:

$$
\begin{gathered}
\left\{Q_{\alpha}, Q_{\beta}^{\dagger}\right\}=-2\left(P_{L} \gamma^{\mu} \gamma^{0}\right)_{\alpha \beta} P_{\mu} \\
\left\{Q_{\alpha}, Q_{\beta}\right\}=\left[Q_{\alpha}, P_{\mu}\right]=0=\left[P_{\mu}, P_{\nu}\right]
\end{gathered}
$$

where $P^{\mu}$ is the energy momentum vector and $P_{L}=\left(1-\gamma^{5}\right) / 2$. Eqs. $(9,10)$ are referred to as a "Graded Lie Algebra". One has that $M^{2} \equiv-P^{\mu} P_{\mu}$ is a Casimir operator and so all states in a SUSY multiplet have the same mass. One may now verify that each such multiplet has an equal number bose and fermi states: Define the Witten index $(-1)^{N}$, where $N_{f}$ is the operator whose eigenvalues are number of fermions in a state. Since $Q_{\alpha}$ is a fermionic operator, it must anti-commute with $(-1)^{N /}$. Hence if $T r$ is the trace over the states of a multiplet we have

$$
\begin{aligned}
\operatorname{Tr}\left[(-1)^{N_{s}}\left\{Q_{\alpha}, Q_{\beta}^{\dagger}\right\}\right] & =\operatorname{Tr}\left[-Q_{\alpha}(-1)^{N_{s}} Q_{\beta}^{\dagger}+(-1)^{N_{s}} Q_{\beta}^{\dagger} Q_{\alpha}\right. \\
& =0
\end{aligned}
$$

But by Eq. (9) this implies

$$
O=\operatorname{Tr}(-1)^{N_{s}} P_{\mu}=P_{\mu}^{\prime} \operatorname{Tr}(-1)^{N_{t}}
$$

where $P_{\mu}^{\prime}$ is the (common) eigenvalue of the multiplet. $\operatorname{Thus} \operatorname{Tr}(-1)^{N_{\jmath}}=0$ for $P_{\mu}^{\prime} \neq 0$ and hence the number fermi and bose states must be equal when summed over the multiplet.

The above considerations would be an amusing toy were it not for two remarkable theorems. The first of these is the following [7]: Aside from an irrelevant generalization [8], the only graded algebra for an $S$-matrix constructed from a local relativistic quantum field theory is the supersymmetry algebra. Thus supersymmetry is unique i.e. it is the only graded extension of Lorentz covariant field theory.

We mention now several special features of SUSY systems. Taking the trace of Eq. (9) gives

$$
P^{0} \equiv H=\frac{1}{4}\left(Q Q^{\dagger}+Q^{\dagger} Q\right) \geq 0
$$

i.e. the Hamiltonian is always a positive semi-definite operator. If the vacuum state is supersymmetric, i.e. $Q_{a}\left|0>=0=Q_{a}^{+}\right| 0>$ then the vacuum energy vanishes: $E_{v a c}=\langle 0|H| 0\rangle \equiv 0$. If there is spontaneous breaking of supersymmetry, i.e. $Q_{a}|0\rangle \neq$ 0 and $Q_{\alpha}^{+} \mid 0>\neq 0$, then $E_{v a c}=<0|H| 0>>0$. This suggests that it may be difficult to break supersymmetry as the symmetric vacuum always lies lower than the broken vacuum. To break global SUSY one must arrange it so that the symmetric vacuum 
does not exist (i.e. is not an extrema of the effective potential). In fact, for a wide class of systems it can be shown that if SUSY doesn't break at the tree level, it doesn't break with quantum corrections [9].

The simplest SUSY multiplets are the massless ones which consist of states of spin $s$ and $s+1 / 2$. For model building, we will need the following:

(i) Chiral multiplet: $(z(x), \chi(x))$

This consists of $z(x)$, a complex scalar field $(s=0)$, and $\chi(x)$ a $L$ Weyl spinor. There are thus 2 bose and 2 fermi states in this multiplet. These multiplets can be used to represent matter, since quarks and leptons are represented by $L$ Weyl spinors. The $z(x)$ fields are additional spin zero fields needed for supersymmetry-the "squarks" and "sleptons".

(ii) Vector multiplet: $\left(V^{\mu}(x), \lambda(x)\right)$

This consists of $V^{\mu}(x)$, a real vector field $(s=1)$ and $\lambda(x)$ a Majorana spinor $(s=1 / 2)$. Again there are 2 bose and 2 fermi states (a massless vector boson has only 2 helicity states). This multiplet can be used to represent gauge bosons, the additional spinor $\lambda(x)$ being the supersymmetric "gaugino" partner.

The dynamics of global SUSY consists of supersymmetrized gauge and Yukawa interactions:

Gauge Interactions: Here there are three types of terms: (a) the bose gauge interactions obtained in the usual way by the replacement

$$
\partial_{\mu} \rightarrow \partial_{\mu}-i \sum_{i} g_{i} V_{\mu i}^{a} T_{i}^{a}
$$

where $V_{\mu i}^{a}, g_{i}$ and $T_{i}^{a}$ are the gauge bosons, gauge coupling constants and group representations for the sub-group $G_{i}$. (The full symmetry group may be a product group $G=\prod_{i} G_{i}$ ); (b) the fermi gaugino interactions with the chiral multiplets $\left(z_{m}(x), \chi_{m}(x)\right)$,

$$
\mathcal{L}_{\lambda}=-i \sqrt{2} \sum_{i, m} g_{i} \bar{\lambda}_{i}^{a} z_{m}^{\dagger} T_{i}^{a} \chi_{m}
$$

and (c) an additional bose contribution to the effective potential, the " $D$ term",

$$
V_{D}=\frac{1}{2} \sum_{i, \alpha} g_{i}^{2} D_{i}^{a} D_{i}^{a} ; D_{i}^{a}=\sum_{m} z_{m}^{\dagger} T_{i}^{a} z_{m}
$$

Together, the interactions of Eqs. $(14,15,16)$ are supersymmetric and gauge invariant.

Yukawa Interactions: These are governed by a superpotential $W\left(z_{m}\right)$. (Note $W$ is "holomorphic" in the sense it is a function of the $z(x)$ but not the $z^{\dagger}(x)$.) The bose Yukawa interactions are the " $F$ term" contribution to the effective potential

$$
V_{F}=\sum_{m}\left|\frac{\partial W}{\partial z_{m}}\right|^{2},
$$


and the fermi Yukawa interactions are given by the Lagrangian term,

$$
\mathcal{L}_{Y}=-\frac{1}{2} \sum_{m, n} \bar{\chi}_{m}^{C} \frac{\partial^{2} W}{\partial z_{m} \partial z_{n}} \chi_{n}+\text { h.c. }
$$

where $\chi^{C}$ means charge conjugate field. Again Eqs. $(17,18)$ together are supersymmetric.

Thus, given a gauge invariant superpotential $W$ and the gauge group $G$, Eqs. (14)-(18) are a unique set of supersymmetric gauge invariant interactions.

The above statement of supersymmetric dynamics leads to the second remarkable theorem of supersymmetry i.e. supersymmetric systems as described above have no quadratic divergences. More precisely, there is no renormalization at all for any of the couplings in the $F$ term (i.e. in $V_{Y}$ ). The only infinities of the theory are logarithmic infinities of wave function renormalizations and gauge coupling constant renormalizations [10]. Thus, not only is SUSY the unique graded extension of the SM [7], but it also eliminates the quadratic Higgs self mass divergence (which was the most serious theoretic disability of the SM).

One can see how this comes about as follows. Not only does the Higgs have the usual interactions with quarks of the SM, but also, from $V_{Y}$, a squark interaction is required to maintain supersymmetry. This is shown in Fig. 2. Both diagrams are quadratically divergent but with opposite sign. With perfect supersymmetry all divergences cancel! When supersymmetry is broken, the quark and squark masses are no longer degenerate and a logarithmic divergence survives. The cutoff $\Lambda^{2}$ of Eq. (8) gets replaced, $\Lambda^{2} \rightarrow\left(m_{\bar{q}}^{2}-m_{q}^{2}\right) \ln \left(\Lambda^{2} / m_{\bar{q}}^{2}\right)$. To avoid fine tuning we now require $m_{\tilde{q}} \lesssim 1 \mathrm{TeV}$. Thus we expect the new SUSY particles to be within striking distance of current and planned accelerators.
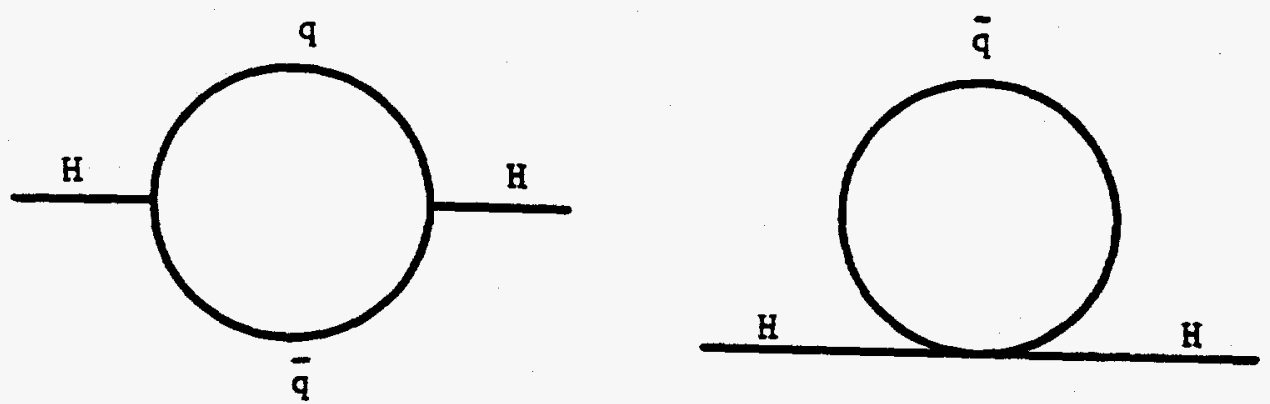

Figure 2: Higgs one loop corrections in supersymmetric models. Compare with the Standard Model diagram of Fig. 1.

\section{Minimal Supersymmetric Standard Model (MSSM)}

The MSSM is the simplest supersymmetric extension of the Standard Model. One promotes each particle in the SM to either a chiral or vector supermultiplet. 
The particle content is given in Table 1. Unique to the MSSM is the appearance now of two Higgs doublets, $H_{1}$ and $H_{2}$. Higgs doublets must come in pairs in SUSY models in order to cancel anomalies. Thus the Higgs boson has become a chiral multiplet, implying the existance now of the fermion Higgsinos, and hence to the danger of possible anomalies. (Anomalies only arise in fermionic triangle loops and so the SM does not have this problem.) A necessary condition to cancel anomalies is that $\sum Y_{i}=0$ where $Y$ is the hypercharge. One sees that $Y_{H_{1}}=-1$ and $Y_{H_{2}}=+1$ so that the anomaly cancellation condition is obeyed when both Higgs doublets are present. (Anomalies in the quark and lepton sector of course cancel by the usual GIM mechanism).

\begin{tabular}{|c|c|}
\hline \multicolumn{2}{|c|}{ Vector Multiplets } \\
\hline $\begin{array}{c}g_{\mu}^{a}(x), a=1 \cdots 8 \\
\text { gluons }(g) \\
B_{\mu}^{\alpha}(x), B_{\mu}^{Y}(x), \alpha=1,2,3 \\
S U(2)_{L} \times U(1)_{Y} \text { gauge bosons } \\
\end{array}$ & $\begin{array}{c}J=\overline{2} \\
\lambda^{a}(x), a=1 \cdots 8 \\
\text { gluinos }(\tilde{g}) \\
\lambda^{\alpha}(x), \lambda^{Y}(x), \alpha=1,2,3 \\
S U(2)_{L} \times U(1)_{Y} \text { gauginos } \\
\end{array}$ \\
\hline \multicolumn{2}{|c|}{ Chiral Multiplets } \\
\hline$j=\frac{1}{2}$ & $j=0$ \\
\hline $\begin{array}{c}\left(u_{i L}, d_{i L}\right) ; u_{i R}, d_{i R} \\
\text { quarks }(i=\text { generation }) \\
\left(\nu_{i L}, e_{i L}\right) ; e_{i R} \\
\text { leptons } \\
\tilde{H}_{1}=\left(\tilde{H}_{1}^{\circ}, \tilde{H}_{1}^{-}\right) ; \tilde{H}_{2}=\left(\tilde{H}_{2}^{+}, \tilde{H}_{2}^{0}\right) \\
\text { Higgsinos }\end{array}$ & $\begin{array}{c}\left(\tilde{u}_{i L}, \tilde{d}_{i L}\right) ; \tilde{u}_{i R}, \tilde{d}_{i R} \\
\text { squarks } \\
\left(\tilde{\nu}_{i L}, \tilde{e}_{i L}\right) ; \tilde{e}_{i R} \\
\text { sleptons } \\
\left.H_{1}=H_{1}^{0}, H_{1}^{-}\right) ; H_{2}=\left(H_{2}^{+}, H_{2}^{0}\right) \\
\text { Higgs bosons }\end{array}$ \\
\hline
\end{tabular}

Table 1. Particle content of the MSSM.

The Yukawa couplings are obtained from the superpotential. From Eqs. $(17,18)$, renormalizability and gauge invariance imply that $W$ is at most a sum of quadratic and cubic terms,

$$
W=W^{(2)}+W^{(3)},
$$

and the general form that preserves baryon number $(B)$ and lepton number $(L)$ and is $S U(3)_{C} \times S U(2)_{L} \times U(1)_{Y}$ invariant is [11]

$$
\begin{gathered}
W^{(2)}=\mu H_{1}^{\alpha} H_{2 \alpha} \\
W^{(3)}=\lambda_{i j}^{(u)} \widetilde{q}_{i}^{\alpha} H_{2 \alpha} \tilde{u}_{j}^{C}+\lambda_{i j}^{(d)} \widetilde{q}_{i}^{\alpha} H_{1 \alpha} \tilde{d}_{j}^{C} \\
+\lambda_{i j}^{(e)} \tilde{\ell}_{i}^{\alpha} H_{1 \alpha} \tilde{e}_{i}^{C}
\end{gathered}
$$


where $\lambda_{i j}^{(u, d, e)}$ are the Yukawa coupling constants as in Eq. (5). $\mu$ is a parameter with dimensions of mass. Note that in the SM with only one Higgs doublet, $B$ and $L$ are automatically conserved. In SUSY, however, this is not the case as $H_{1}$ has the same quantum numbers as the lepton doublets $\ell_{i}$. Thus it is possible, for example, to violate $L$ by adding to Eq. (20) a term $\ell_{i}^{\alpha} H_{2 \alpha}$ [and similarly replace $H_{1}$ by $\ell_{i}$ in Eq. (21)]. We will not consider such models here.

$W^{(2)}$ is a general mixing term between the two Higgs doublets and is scaled by the mass parameter $\mu$, while $W^{(3)}$ are the Yukawa couplings. When $S U(2) \times U(1)$ breaks, one expects, in general, both neutral components, $H_{1}^{0}$ and $H_{2}^{0}$, to grow VeVs. Thus one sees that $\left\langle H_{2}^{0}\right\rangle$ will give rise to $u$-quark masses, and $\left\langle H_{1}^{0}\right\rangle$ to d-quark and lepton masses. Further, one needs two distinct Higgs doublets to form the Yukawa interactions of Eq. (21) e.g. one could not use $H_{1}$ in the $u$-quark term as it has the wrong hypercharge. One would need to use $H_{1}^{\dagger}$ to form an $u$-quark Yukawa term. But supersymmetry requires $W$ to be holomorphic, i.e. a function of the scalar fields $z_{m}$ only and not of $z_{m}^{\dagger}$. Hence a term with $H_{1}^{\dagger}$ is forbidden. Thus, two Higgs doublets are needed in supersymmetry both on fundamental grounds (to cancel anomalies) and on phenomenological grounds (so that both $u$ and $d$ quarks can grow masses), a neat matching of rather disparat requirements.

After $S U(2) \times U(1)$ breaks, there is a mixing of Higgsinos and $S U(2) \times U(1)$ gauginos. Thus the $\mathrm{H}_{2}$ part of Eq. (15) for the $S U(2)$ gauginos is

$$
\begin{aligned}
\mathcal{L}_{\lambda}= & -i \sqrt{2} g_{2} \bar{\lambda}^{i} H_{2}^{\alpha \dagger}\left(\frac{\tau^{i}}{2}\right)_{\alpha \beta} \tilde{H}_{2}^{\beta}+\text { h.c. } \\
& \rightarrow-i \sqrt{2} g_{2} \bar{\lambda}^{i}<H_{2}^{\alpha}>^{*}\left(\frac{\tau^{i}}{2}\right)_{\alpha \beta} \tilde{H}_{2}^{\beta}
\end{aligned}
$$

Also, from Eq. (18) $W^{(2)}$ gives a mixing between $\tilde{H}_{1}$ and $\tilde{H}_{2}$ :

$$
\begin{aligned}
\mathcal{L}_{Y}= & -\frac{1}{2} \tilde{\bar{H}}_{1}^{\alpha c} \frac{\partial^{2} W}{\partial H_{1}^{\alpha} H_{2 \beta}} \tilde{H}_{2 \beta}+\text { h.c. } \\
& \rightarrow-\mu \bar{H}_{1}^{\alpha} H_{2 \alpha}
\end{aligned}
$$

Thus one gets a mass matrix coupling Higgsinos and gauginos. The mass diagonal states are the 2 charginos (Winos) $\tilde{W}_{i}, i=1,2$ (charged spin 1/2 Dirac fields) and 4 neutralinos (Zinos) $Z_{i}, i=1 \cdots 4$ (neutral spin 1/2 Majorana fields). (We label our states such that $m_{i}<m_{j}$ for $i<j$.). The $W^{ \pm}$and $Z^{0}$ bosons grow masses by the usual Higgs mechanism, absorbing a charged Higgs field and a hermitian neutral Higgs field from the $H_{1}$ and $H_{2}$ doublets. Thus there is left 3 neutral and one charged Higgs bosons which we denote as follows: $h^{0}$ and $H^{\circ}$ (CP even states), $A^{\circ}$ (CP odd state) and $H^{ \pm}$(charged state). The $h^{0}$ is defined to be the Higgs boson that most closely resembles the SM Higgs.

In summary then, the MSSM implies the existance of 32 SUSY particles (over and above the usual quarks and leptons of the SM): 12 squarks, 9 sleptons, 2 Winos, 4 Zinos, 1 gluino and 4 Higgs bosons. 
There still remains, however, the problem of supersymmetry breaking. In a supersymmetric multiplet, the bose and fermi states all have the same mass. Thus, for example, one a priori expects the squarks to be degenerate with the quarks. However, no light bosons of this type exist experimentally, which must mean that supersymmetry is a broken symmetry if it is to have any validity. The only satisfactory way of breaking a symmetry is by spontaneous breaking. As we saw, however, supersymmetry is very resistent to breaking, and no physically acceptable way of producing spontaneous breaking is known for global supersymmetry. One is thus reduced to adding, on purely phenomenological grounds, symmetry breaking terms in order to construct a viable model. However, one must do this in a fashion that still maintains the cancellation of the quadratic divergences. Such type of SUSY symmetry breaking is called "soft breaking" and only a limited number of symmetry breaking terms will do this [12].

The general form of soft breaking terms that one can add to the supersymmetrized Standard Model is a contribution to the effective potential of the form

$$
\begin{aligned}
V_{S B} & =m_{a b}^{2} z_{a} z_{b}^{\dagger}+\left[A_{i j}^{(u)} \lambda_{i j}^{(u)} q_{i} H_{2} u_{j}^{C}+A_{i j}^{(d)} \lambda_{i j}^{(d)} q_{i} H_{1} d_{j}^{C}\right. \\
& \left.+A_{i j}^{(e)} \lambda_{i j}^{(e)} \ell_{i} H_{1} e_{j}^{C}+B \mu H_{1} H_{2}+h . c .\right]
\end{aligned}
$$

and a gaugino mass term

$$
\mathcal{L}_{\text {mase }}^{\lambda}=-\bar{m}_{i} \bar{\lambda}^{i} \lambda^{i}
$$

Here $\left\{z_{a}\right\}$ are the set of scalar fields of the chiral multiplets and $\left\{\lambda^{i}\right\}$ are all the gauginos. Thus $m_{a b}^{2}$ is the scalar mass matrix, $A_{i j}^{(u, d, e)}$ and $B$ are soft breaking constants (sometimes called "Polonyi" constants) and $\tilde{m}_{i}$ are the gaugino Majorana masses [13]. This general form of the soft breaking terms then depends on 137 parameters (or 87 if one assumes them all real)! Many, of course, can be eliminated on purely phenomenological grounds, but there would still be too many for the model to have much predictive value. Instead, a limited number are assumed to exist in the so called "Minimal Supersymmetric Standard Model" (MSSM) which we now define by the following conditions:

(i) The particle content is that of the supersymmetrized Standard Model with one pair of Higgs doublets (no extra "exotic" particles).

(ii) All squarks (except perhaps the t-squarks) are degenerate. All sleptons are degenerate.

(iii) Gaugino masses $\tilde{m}_{i}, i=1,2,3$ for $U(1)_{Y}, S U(2)_{L}, S U(3)_{C}$ obey

$$
\bar{m}_{Y}: \tilde{m}_{2}: \tilde{m}_{3}=\alpha_{Y}: \alpha_{2}: \alpha_{3}
$$

These assumptions greatly reduce the number of free parameters and make the theory phenomenologically useful. Further, as we will see later, the MSSM is an 
approximation to the low energy limit of supergravity Gut models (and in fact was constructed after the invention of the supergravity models).

\section{Experimental Bounds on SUSY Masses}

Experimental searches for SUSY particles have been on going for a decade now. Current bounds come from the Tevatron (CDF) and from the absence of SUSY particles in $Z$ decays at LEP. We list these bounds now for reference. All analysis of experimental data has been done with the framework of the MSSM.

Lower bounds on the gluino mass $m_{\tilde{g}}$ and on the squark mass $m_{\tilde{q}}$ are difficult to state as the bounds on the two particles are correlated and the bounds also depend on the parameters $\mu$ and $\left.\tan \beta \equiv<H_{2}\right\rangle /\left\langle H_{1}\right\rangle$. At present, a full analysis of the CDF data has not been made but roughly one may say $m_{\tilde{q}}, m_{\tilde{\mathbf{g}}} \gtrsim 100 \mathrm{GeV}$ if $m_{\tilde{g}} \lesssim 400 \mathrm{GeV}$. Fig. 3 shows the parameter choice that has been analysed [14]. The excluded regions may change as $\mu$ and $\tan \beta$ are varied.

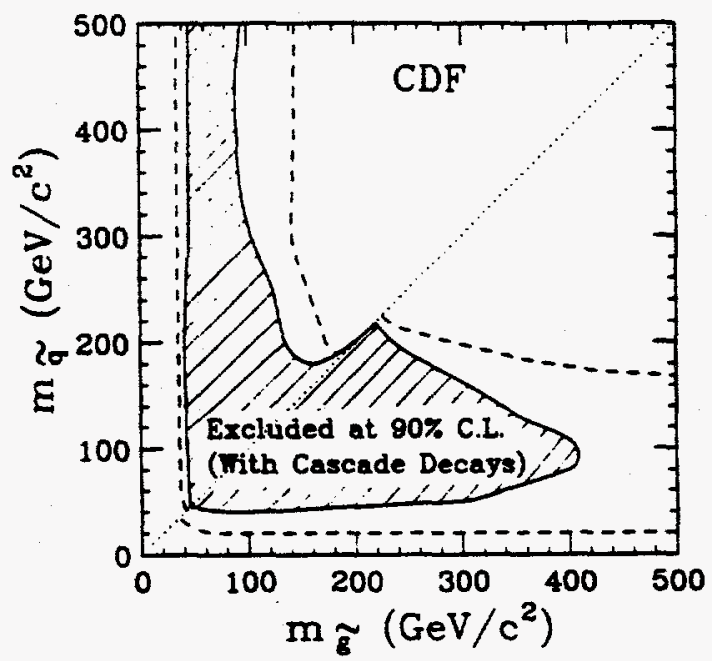

Figure 3: Excluded region in $m_{\tilde{g}}-m_{\tilde{q}}$ for $\mu=-250 \mathrm{GeV}, \tan \beta=2$ [CDF].

Bounds on the other SUSY particles come from LEP [15]. Since SUSY particles are created in pairs, the absence of them in $Z$ decay generally gives a lower bound of approximately $M_{Z} / 2 \simeq 45 \mathrm{GeV}$ (unless the coupling to the $Z$ is anomalously small). One finds, in fact for the charged sleptons that $m_{\tilde{e}}, m_{\tilde{\mu}}, m_{\tilde{\tau}}>45 \mathrm{GeV}$ and $m_{\bar{\nu}}>42 \mathrm{GeV}$. The charginos and neutralinos are bounded by $m_{\tilde{W}_{1}}>45 \mathrm{GeV}$, and $m_{\tilde{z}_{1}}>20 \mathrm{GeV}$ and $m_{\tilde{z}_{2}}>45 \mathrm{GeV}$ for $\tan \beta>3$. The Higgs boson bounds are $m_{h}>43 \mathrm{GeV}, m_{A}>20-44 \mathrm{GeV}, m_{H^{ \pm}}>42 \mathrm{GeV}$. However, if $m_{A}$ is large $\left(m_{A}^{2} \gg M_{Z}^{2}\right)$, the $h$ boson couplings are similar to the Standard Model Higgs and the bound rises to $m_{h}>62.5 \mathrm{GeV}$.

One sees that if we expect the new SUSY particles to inhabit a domain 
from somewhat below $M_{Z}$ up to about $1 \mathrm{TeV}$, the parameter space has not yet been much explored, and it is not too surprising that SUSY particles have not yet been discovered. In the near future, some of these bounds can be increased. Thus LEP200, with maximum luminosity and energy, should be able to detect the $h$ boson with mass up to $95 \mathrm{GeV}$, and the $\tilde{W}_{1}$ up to $100 \mathrm{GeV}$. The Tevatron, with a data sample of $100 \mathrm{pb}^{-1}$, could detect squarks (except the $\tilde{t}_{1}$ ) up to about $200 \mathrm{GeV}$, the $\tilde{t}_{1}$ up to about $100 \mathrm{GeV}$ and the $\tilde{W}_{1}$ up to about $70 \mathrm{GeV}$ (and perhaps higher in some models). The LHC and SSC will be able to detect the gluino and squarks up to $2 \mathrm{TeV}$, as well as be able to see the lighter Wino, Zinos and $h$ boson.

\section{Unification of Couplings}

About three years ago it became possible to test the question of whether there is unification of the $S U(3)_{C}, S U(2)_{L}$, and $U(1)_{Y}$ coupling constants at a high mass scale. This was a result of accurate measurements of $\alpha_{1}, \alpha_{2}$ and $\alpha_{3}$ at the $Z$ mass scale. It is convenient to use the fine structure constant $\alpha\left(M_{Z}\right)$ and $\left(\mathrm{sm}^{2} \theta_{W}\right)_{m s}$ as input to determine $\alpha_{1} \equiv 5 \alpha / 3 \cos ^{2} \theta_{W}$ and $\alpha_{2}=\alpha / \sin ^{2} \theta_{W}$. Current determinations are $[16,17]$

$$
\begin{gathered}
\alpha^{-1}\left(M_{Z}\right)=127.9 \pm 0.1 \\
\left(\sin ^{2} \theta_{W}\right)_{\overline{m z}}=0.2328 \pm 0.0007
\end{gathered}
$$

Note that $\alpha\left(M_{Z}\right)$ is much less accurately known then its value at the Thompson limit, $\alpha^{-1}\left(m_{e}\right)=137.0359895(61)$. This is due to lack of data in the (1-10) $\mathrm{GeV}$ region in $e^{+} e^{-}$scattering needed to accurately run $\alpha$ from $m_{e}$ to $M_{Z}$. In fact, the error in $\alpha\left(M_{Z}\right)$ is the major error arising in high precision tests of the electroweak sector of the Standard Model. One finds from Eqs. (27), (28) for $\alpha_{1,2}\left(M_{Z}\right)$ then

$$
\begin{gathered}
\alpha_{1}\left(M_{Z}\right) \equiv(5 / 3) \alpha_{Y}=0.016985 \pm 0.000020 \\
\alpha_{2}\left(M_{Z}\right)=0.03358 \pm 0.00011
\end{gathered}
$$

and the World average for $\alpha_{3}\left(M_{Z}\right)$ is [18]

$$
\alpha_{3}\left(M_{Z}\right)=0.118 \pm 0.007
$$

Using the Renormalization Group Equations (RGE) one can determine the $\alpha_{i}$ at any other mass scale and see whether or not they meet at some high scale $\mu=M_{G}$. The RGE to 2-loop order for $\alpha_{i}(\mu), i=1,2,3$ are [19]

$$
\mu \frac{d \alpha_{i}(\mu)}{d \mu}=\frac{1}{2 \pi}\left[b_{i}+\frac{1}{4 \pi} \sum_{j} b_{i j} \alpha_{j}(\mu)\right] \alpha_{i}^{2}(\mu)
$$

where for the Standard Model, $b_{i}$ and $b_{i j}$ are 


$$
\begin{gathered}
b_{i}=(0,-22 / 3,-11)+N_{F}(4 / 3,4 / 3,4 / 3)+N_{H}(1 / 10,1 / 6,0) \\
b_{i j}=\left(\begin{array}{ccc}
0 & 0 & 0 \\
0 & -136 / 3 & 0 \\
0 & 0 & -102
\end{array}\right)+N_{F}\left(\begin{array}{ccc}
19 / 15 & 3 / 5 & 44 / 15 \\
1 / 5 & 49 / 3 & 4 \\
11 / 30 & 3 / 2 & 76 / 3
\end{array}\right)+N_{H}\left(\begin{array}{ccc}
9 / 50 & 9 / 10 & 0 \\
3 / 10 & 13 / 6 & 0 \\
0 & 0 & 0
\end{array}\right)
\end{gathered}
$$

For the MSSM one has

$$
\begin{gathered}
b_{i}=(0,-6,-9)+N_{F}(2,2,2)+N_{H}(3 / 10,1 / 2,0) \\
b_{i j}=\left(\begin{array}{ccc}
0 & 0 & 0 \\
0 & -24 & 0 \\
0 & 0 & -54
\end{array}\right)+N_{F}\left(\begin{array}{ccc}
38 / 15 & 6 / 5 & 88 / 15 \\
2 / 5 & 14 & 8 \\
11 / 15 & 3 & 68 / 3
\end{array}\right)+N_{H}\left(\begin{array}{ccc}
9 / 50 & 9 / 10 & 0 \\
3 / 10 & 7 / 2 & 0 \\
0 & 0 & 0
\end{array}\right)
\end{gathered}
$$

In Eqs. (33-36) $N_{F}$ is the number of families $\left(N_{F}=3\right)$ and $N_{H}=$ the number of Higgs doublets. ( $N_{H}=1$ for the SM and $N_{H}=2$ for the MSSM). The difference between the $b_{i}$ and $b_{i j}$ of the SM and the MSSM is due, of course, to the different particle spectrum.

Using the experimental values of $\alpha_{i}\left(M_{Z}\right)$ as initial conditions, one may check whether the $\alpha_{i}$ meet at $\mu=M_{G}$ :

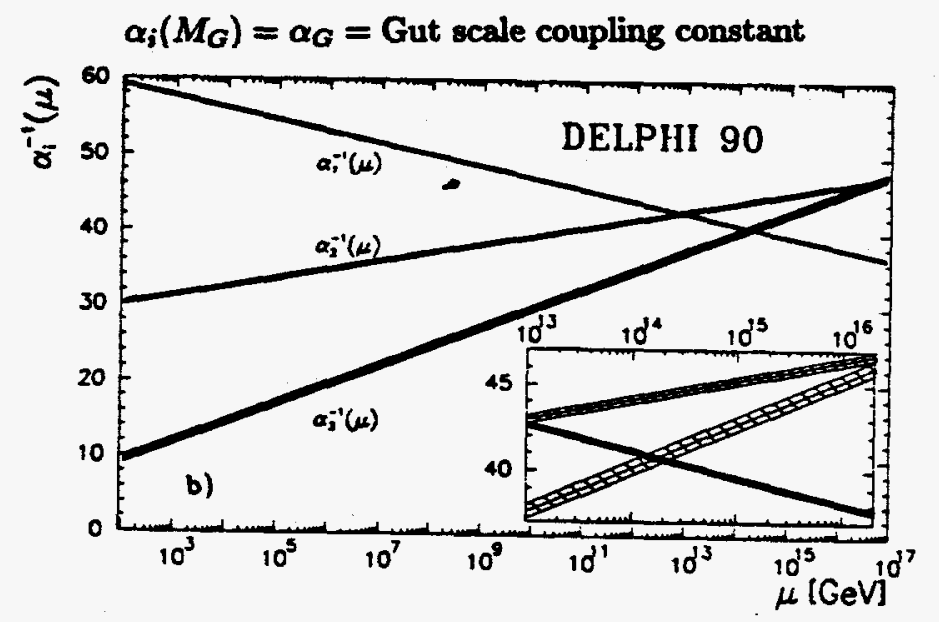

Figure 4: $\alpha_{i}^{-1}(\mu)$ for the Standard Model with 1 Higgs doublet. The three coupling constants do not meet.

For the SM, grand unification fails by more than 7 std (Fig. 4 Amaldi et al [2]). For supersymmetry one may consider the simplest approximation of assuming all SUSY particles are degenerate at a common mass $M_{S}$. Then acceptable unification does occur but only for one pair of light Higgs doublets (Fig. 5, Fig. 6 Amaldi et al [2]). A fit to the data for the MSSM depends on the value of $\alpha_{3}\left(M_{Z}\right)$. One finds $\alpha_{G}^{-1}=25.4 \pm 1.7$ and 


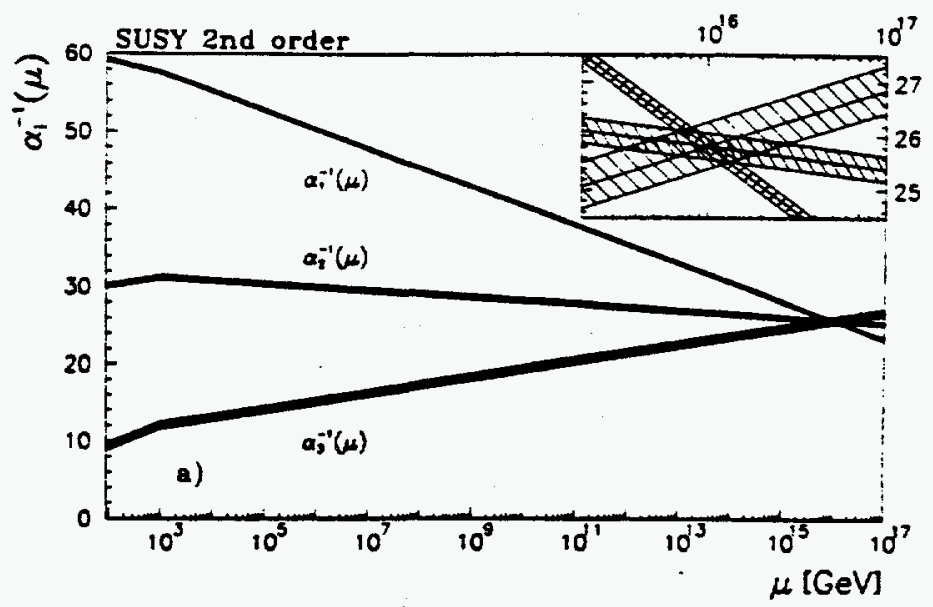

Figure 5: $\alpha_{i}(\mu)$ for the MSSM with one pair of Higgs doublets showing grand unification.

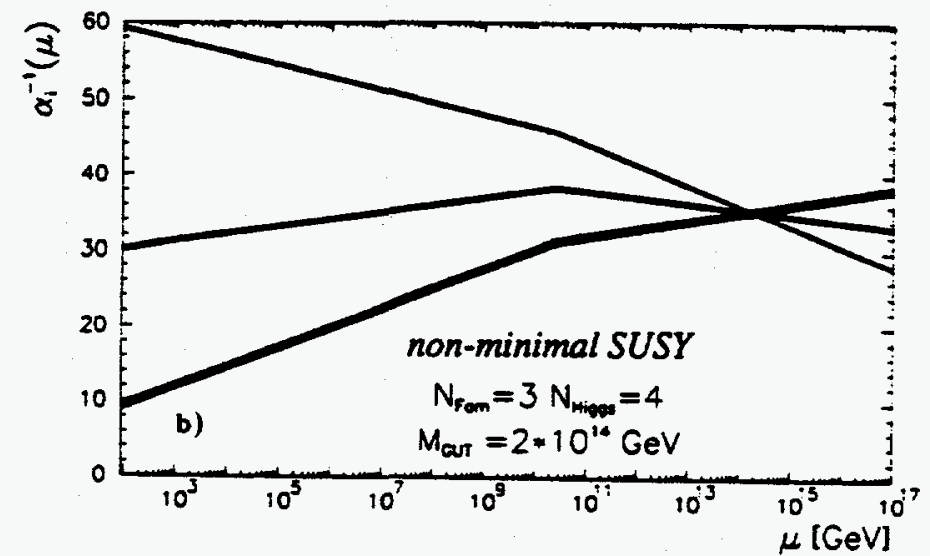

Figure 6: $\alpha_{i}(\mu)$ for the MSSM with two pairs of Higgs doublets. While unification occurs, $M_{G}$ is too small to prevent rapid proton decay $p \rightarrow e^{+} \pi^{0}$ and $M_{S}$ is too large to resolve the gauge hierarchy problem.

$$
\begin{aligned}
& M_{G} \cong 10^{16.2+5.7\left(\alpha_{2} / 0.118-1\right)} \\
& M_{S} \cong 10^{2.4+17.4\left(1-\alpha_{2} / 0.118\right)}
\end{aligned}
$$

We see that there is an anti-correlation between $M_{G}$ and $M_{S}$ :

$$
M_{G} \cong 10^{16.2}\left[10^{2.4} / M_{S}(\mathrm{GeV})\right]^{0.33}
$$

i.e a larger $M_{S}$ gives a smaller $M_{G}$, and from Eq. (39) a larger $M_{S}$ corresponds to a smaller value of $\alpha_{3}$.

We note the following points concerning the above results:

(i) The major experimental error in the analysis is due to the experimental error in $\alpha_{3}\left(M_{Z}\right)$. Last year there appeared to be the possibility of a discrepency between the low energy evaluations of $\alpha_{3}\left(M_{Z}\right)$ and the high energy LEP results. Recently, however, the LEP average has been lowered to [20] $\alpha_{s}\left(M_{Z}\right)=0.123 \pm 0.006$ while 
the deep inelastic scattering evaluation is $\alpha_{3}\left(M_{z}\right)=0.112 \pm 0.005$. Thus the choice of Eq. (31) is in reasonable agreement with both types of determinations.

(ii) It is $\alpha_{1} \equiv(5 / 3) \alpha_{Y}$ that unifies with $\alpha_{2}$ and $\alpha_{3}$. From low energy SUSY (e.g. the MSSM) this looks mysterious, i.e. why a $5 / 3$ factor? However, from Gut physics based on $S U(5), S O(10)$ and other groups this is just what is expected, i.e. the unification should occur when the hypercharge is embedded in the grand unified group with a factor $5 / 3$. This implies that the unification of the couplings is not just a property of low energy SUSY, but also requires information about Gut scale physics, i.e. the MSSM by itself does not imply grand unification.

(iii) The SUSY masses are, of course, not all degenerate and one needs to include SUSY mass splitting. There are also similar mass thresholds at the Gut scale and these can also modify the results. However, one cannot discuss this within the framework of the MSSM as one needs a Gut model. We will come back to this within the framework of supergravity grand unification.

(iv) Unification of couplings does not appear to be good news for string theory which also expects unification, but at the Planck scale $M_{P t}=2.4 \times 10^{18} \mathrm{GeV}$. Two possible explanations for this discrepency have been proposed: There may be string threshold effects that modify the unification point. Alternately, the string model (vacuum state) may predict the existance of "exotic" particles with masses (less than $M_{G}$ ) of just the right values so that the (modified) RGE delay unification until $M_{P \ell}$. However, up to now, no string model that predicts the desired properties has been constructed.

\section{Local Supersymmetry (Supergravity)}

In order to obtain a phenomenologically acceptable spontaneous breaking of supersymmetry, it appears to be necessary to promote supersymmetry from a global to a local symmetry (which theoretically is a natural thing to do). This turns out to require that gravity be included into the analysis, i.e. local supersymmetry is supergravity. We give in this section a brief discussion of the ideas of supergravity.

To see why local supersymmetry forces one to include gravity, consider the simplest global SUSY system of a non-interacting chiral multiplet, $\{z(x), \chi(x)\}$. The Lagrangian is [6]:

$$
\mathcal{L}=-\partial_{\mu} z^{\dagger} \partial^{\mu} z-\bar{\chi}(-i) \gamma^{\mu} \partial_{\mu} \chi
$$

$\mathcal{L}$ is invariant under the global SUSY transformation

$$
\delta z(x)=\bar{\varepsilon} \chi(x) ; \delta \chi(x)=-i \gamma^{\mu}\left[\partial_{\mu} z(x)\right] \varepsilon
$$

where $\varepsilon$ is an infinitesimal anti-commuting constant spinor. Suppose now we replace $\varepsilon$ by $\varepsilon(x)$. Now $\mathcal{L}$ is no longer invariant i.e. 


$$
\delta \mathcal{L}=\left[\partial_{\mu} \bar{\varepsilon}(x)\right]\left(\gamma^{\mu}\left[\gamma^{\alpha} \partial_{\alpha} z(x)\right] \chi\right)+\text { divergence }
$$

To compensate for this term, we proceed as usual by introducing a compensating gauge field (the "gravitino")

$$
\psi_{\alpha}^{\mu}(x)=\operatorname{spin} 3 / 2 \text { field }
$$

( $\alpha=$ spinor index) with transformation rule

$$
\delta \psi^{\mu}=\kappa^{-1} \partial^{\mu} \varepsilon(x) ;[\kappa]=(\text { mass })^{-1}
$$

We add to $\mathcal{L}$ the interaction term

$$
\mathcal{L}_{1}=-\kappa \bar{\psi}_{\mu} \gamma^{\mu}\left[\gamma^{\alpha} \partial_{\alpha} z(x)\right] x
$$

which clearly cancels the $\partial_{\mu} \bar{\varepsilon}(x)$ term of Eq. (43) under the combined transformations of Eqs. (42) and (45). However, $\mathcal{L}+\mathcal{L}_{1}$ is still not supersymmteric, since one finds

$$
\delta \mathcal{L}_{0}+\delta \mathcal{L}_{1}=\kappa \bar{\psi}_{\nu} \gamma_{\mu} T^{\mu \nu} \varepsilon(x)
$$

where $T^{\mu \nu}$ is the $z(x)$ stress tensor. This term can be canceled if we now add to the system a second field,

$$
g_{\mu \nu}(x)=\operatorname{spin} 2 \text { field }
$$

with supersymmetry transformation

$$
\delta g_{\mu \nu}=\kappa \bar{\psi}_{\mu} \gamma_{\nu} \varepsilon(x)
$$

and add the coupling

$$
\mathcal{L}_{2}=-g_{\mu \nu} T^{\mu \nu}
$$

Thus the requirement of local supersymmetry leads to a gauge theory based on the SUSY massless "supergravity multiplet":

$$
\text { s }=\begin{array}{cc}
\left\{\psi^{\mu}(x),\right. & \left.g_{\mu \nu}(x)\right\} \\
3 / 2, & 2
\end{array}
$$

$g_{\mu \nu}(x)$ is clearly the gravitational field (a massless spin 2 particle), $\mathcal{L}_{2}$ being the gravitational coupling, and one recognizes $\kappa^{-1}$ as the Planck mass: $\kappa^{-1}=\left(8 \pi G_{N}\right)^{-1 / 2} \equiv M_{P \ell}$ (where $G_{N}$ is the Newtonian constant).

Next, let us consider the supergravity multiplet, neglecting coupling to matter. The supergravity Lagrangian is just the sum of the spin 2 plus spin 3/2 Lagrangians. Thus introducing the vierbein $e_{\mu}^{m}(x)$ by

$$
g_{\mu \nu}(x)=e_{\mu}^{m}(x) \eta_{m n} e_{\mu}^{n}(x)
$$


the $N=1$ supergravity Lagrangian can be written as [21]

$$
\mathcal{L}_{S G}=-\frac{1}{2} \kappa^{-2} e R\left(e_{\mu}^{m}, w_{\mu}^{m n}\right)-\frac{1}{2} \varepsilon^{\mu \nu \rho \sigma} \bar{\psi}_{\mu} \gamma^{5} \gamma_{\nu} D_{\rho} \psi_{\sigma}
$$

where $e=\operatorname{det}\left[e_{\mu}^{m}\right], R$ is the curvature scalar, $w_{\mu}^{m n}$ is the spin connection (determined by varying Eq. (53) with respect to $w_{\mu}^{m n}$ ) and $D_{\rho}=\partial_{\rho}+\frac{1}{2} w_{\rho}^{m n} \sigma_{m n}, \sigma_{m n} \equiv \frac{1}{4}\left[\gamma_{m}, \gamma_{n}\right]$ is the covariant derivative. Eq. (53) is invariant under the local supersymmetry transformations

$$
\delta e_{\mu}^{m}=\frac{1}{2} \kappa \varepsilon(x) \gamma^{m} \psi_{\mu} ; \delta \psi_{\mu}=\kappa^{-1} D_{\mu} \varepsilon(x)
$$

and one finds $\delta w_{\mu}^{m n}=0$. Eq. (54) is just the generalization of Eqs. (45) and (49) to maintain the general coordinate invariance required by the gravitational interactions.

\section{Supergravity Coupling to Matter}

Just as one couples Einstein gravity to matter, one needs to couple supergravity to matter. For physically interesting systems, we saw that matter consists of a number of chiral multiplets (to represent quarks, leptons etc.) and an arbitrary vector multiplet (to represent the gauge particles). The analysis of how to couple the matter multiplets to the supergravity multiplet and maintain supergravity invariance is very complicated [22,23]. We give here only that part of the Lagrangian relative to Gut model building.

The general couplings of $N=1$ supergravity depend upon three functions, the superpotential $\tilde{W}\left(z_{i}\right)$, the Kahler potential $d\left(z_{i}, z_{i}^{\dagger}\right)$ and the gauge kinetic function $f_{\alpha \beta}\left(z_{i}, z_{i}^{\dagger}\right)$. Here the $\left\{z_{i}\right\}$ are the spin zero components of the left handed chiral multiplets. $\left\{z_{i}, \chi_{i}\right\}$, and $\alpha, \beta$ are adjoint representation gauge indices. $\tilde{W}, d$ and $f_{\alpha \beta}$ are hermitain, $\tilde{W}$ and $d$ are gauge singlets and $f_{\alpha \beta}$ is a gauge tensor. Actually, $\tilde{W}$ and $d$ enter in a single combination

$$
\mathcal{G}=-\kappa^{2} d-\ln \left[\kappa^{6} W W^{\dagger}\right]
$$

so the general supergravity Lagrangian really depends only on two functions $f_{\alpha \beta}$ and $\mathcal{G}$. From Eq. (55) one also sees that the theory is invariant under a Kahler transformation

$$
d \rightarrow d-f\left(z_{i}\right)-f^{\dagger}\left(z_{i}\right), W \rightarrow e^{\kappa^{2} f} W
$$

The "Kahler metric" is defined by

$$
g_{j}^{i}=d_{j}^{i} \equiv \frac{\partial^{2} d}{\partial z_{i} z_{j}^{\dagger}}=-\kappa^{-2} \mathcal{G}_{j}^{i}
$$

For the special case $d=\sum_{i} z_{i} z_{i}^{\dagger}$ one has $g_{j}^{i}=\delta_{j}^{i}$ which is a flat Kahler metric. 
The total Lagrangian is quite complicated containing many non-linear terms scaled by factors of $\left(M_{P \ell}\right)^{-n}$. Most of these are negligibly small at energies below the Planck scale. There are five important terms that we list now:

(i) Effective Potential

The effective potential has the form

$$
\begin{aligned}
V & =-\kappa^{-4} e^{-\mathcal{G}}\left[\left(\mathcal{G}^{-1}\right)_{j}^{j} \mathcal{G}_{,} \mathcal{G}^{j}+3\right] \\
& +\frac{g^{2}}{2}\left[\operatorname{Re}\left(f^{-1}\right)_{\alpha \beta}\right] D_{\alpha} D_{\beta}
\end{aligned}
$$

where $\left(\mathcal{G}^{-1}\right)_{j}^{i},\left(f^{-1}\right)_{\alpha \beta}$ are the matrix inverses of $\mathcal{G}_{j}^{, i}, f_{\alpha \beta}$ and $g$ is the gauge coupling constant. The quantity $D_{\alpha}$ is given by

$$
D_{\alpha}=-\kappa^{-2} \mathcal{G}^{, i}\left(T^{\alpha}\right)_{i j} z_{j}
$$

where $T^{\alpha}$ is the group generator. Re-expressing $V$ in terms of $\tilde{W}$ and $d$ gives

$$
V=e^{\kappa d}\left[\left(d^{-1}\right)_{j}^{i}\left(\frac{\partial \tilde{W}}{\partial z_{i}}+\kappa^{2} d_{, j} \tilde{W}\right)\left(\frac{\partial \tilde{W}}{\partial z_{j}}+\kappa^{2} d_{j} \tilde{W}\right)^{\dagger}-3 \kappa^{2}|\tilde{W}|^{2}\right]+V_{D}
$$

where $V_{D}$ is the last term of Eq. (58)

(ii) Scalar Kinetic Energy

The spin zero kinetic energy is given by

$$
-d_{j}^{i}\left(D^{\mu} z_{i}\right)\left(D_{\mu} z_{j}\right)^{\dagger}
$$

where $D_{\mu}$ is the usual gauge covariant derivative. We will consider Kahler metrics where the non-linear terms are scaled by $1 / M_{P \ell}=\kappa ;$

$$
g_{j}^{i}=d_{j}^{i}=c_{j}^{i}+\kappa c_{j k}^{i} z_{k}+\cdots \text {. }
$$

After spontaneous breakings (of the Gut gauge group and supersymmetry) some of the $z_{k}$ fields will grow VEVs. One can write then $g_{j}^{i}=\left\langle g_{j}^{i}\right\rangle+g_{j}^{i^{\prime}}$ where $\left\langle g_{j}^{i^{\prime}}\right\rangle=0$. One may then make unitary and scale transformations on $z_{i}$, $z_{i}^{\dagger}$ which reduces $\left\langle g_{j}^{i}\right\rangle$ to $\delta_{j}^{i}$ and hence the kinetic energy to canonical form i.e.

$$
g_{j}^{i} \rightarrow \delta_{j}^{i}+\kappa c_{j k}^{i_{k}^{\prime}} z_{k}^{\prime} ;<z_{\kappa}^{\prime}>=0
$$

The extra non-renormalizable terms in Eq. (61) are scaled by $\kappa=1 / M_{P \ell}$ and hence negligible below the Planck scale.

A similar analysis holds for the spinor kinetic energy term of the chiral multiplet which has the form $-d_{j}^{i} \bar{\chi}^{i} \gamma^{\mu} \hat{D}_{\mu} \chi^{j}$ where $\hat{D}_{\mu}$ is the gauge and gravitational covariant derivative. The same transformations used in the spin zero sector also reduces the spinor kinetic energy to canonical form.

(iii) Gauge Kinetic Energy

The gauge kinetic energy has the form 


$$
-\frac{1}{4}\left(\operatorname{Re} f_{\alpha \beta}\right) F_{\mu \nu}^{\alpha} F^{\mu \nu \beta}
$$

We consider $f_{\alpha \beta}$ whose non-linear parts are also scaled by $1 / M_{P \ell}$, i.e. $f_{\alpha \beta}=c_{\alpha \beta}+$ $\kappa c_{\alpha \beta i} z_{i}+\cdots$; which after spontaneous breakings takes the form $c_{\alpha \beta}^{\prime}+\kappa c_{\alpha \beta i} z_{i}^{\prime}+\cdots$, $\left\langle z_{i}^{\prime}\right\rangle=0$. One may now make orthorgonal and scale transformations on $F_{\mu \nu}^{\alpha}$ to reduce $f_{\alpha \beta}$ to $f_{\alpha \beta}=\delta_{\alpha \beta}+\kappa c_{\alpha \beta i}^{\prime \prime} z_{i}^{\prime \prime}+\cdots$ and hence the kinetic energy to canonical form. The same transformations simultaneously reduces the gaugino kinetic energy, $-\frac{1}{2}\left(\operatorname{Ref}_{\alpha \beta}\right) \bar{\lambda}^{\alpha} \gamma^{\mu} \hat{D}_{\mu} \lambda^{\beta}$ to canonical form.

(iv) Gaugino "Mass" Term

A term quadratic in the gaugino fields exists in the Lagrangian:

$$
\left[\frac{1}{4} \kappa^{-1} e^{-\mathcal{G} / 2}\left(\mathcal{G}^{-1}\right)_{j}^{j} \mathcal{G}^{j} f_{\alpha \beta, i}^{\dagger}\right] \bar{\lambda}^{\alpha} \lambda^{\beta}
$$

When supersymmetry breaks, we will see that it is possible for the bracket of Eq. (65) to grow a VEV and hence give rise to a gaugino mass.

(v) Gravitino "Mass" Term

A term quadratic in the gravitino mass also exists in the Lagrangian,

$$
\left[\kappa^{-1} e^{-\sigma / 2}\right] \bar{\psi}_{\mu} \sigma^{\mu \nu} \psi_{\nu}
$$

and if supersymmetry breaks, a VEV of the bracket of Eq. (66) gives rise to a gravitino mass.

The effective potential of Eq. (60) is the supergravity generalization of the global SUSY effective potential. Thus if we consider the expansion of Eq. (63) and take the limit $\kappa \rightarrow 0$ (i.e. $M_{P \ell} \rightarrow \infty$ ), the first term of Eq. (60) reduces precisely to $V_{F}$ of Eq. (17) with the notational change $W \rightarrow \tilde{W}$. Similarly, $D_{\alpha}$ of Eq. (59) reduces to

$$
D_{\alpha}=\left(d^{, i}+\kappa^{-2} \tilde{W}, i / \tilde{W}\right)\left(T^{\alpha}\right)_{i j} z_{j}
$$

However, $\tilde{W}^{j i} T_{i j}^{\alpha} z_{j} \equiv 0$ (since $\tilde{W}$ is a gauge singlet) and writing $d^{i}=z_{i}^{\dagger}+\cdots$ one gets in the limit $\kappa \rightarrow 0$ that $D_{\alpha}=z_{i}^{\dagger}\left(T^{\alpha}\right)_{i j} z_{j}$ [24]. With the expansion of $f_{\alpha \beta}=\delta_{\alpha \beta}+\cdots$ discussed below Eq. (64) we see that in the $x \rightarrow 0$ limit $V_{D}$ of Eq. (58) reduces precisely to Eq. (16) of global supersymmetry. Thus global supersymmetry can be viewed as the limit of supergravity when $M_{P \ell} \rightarrow \infty$.

However, Planck scale effects are crucial in that they allow spontaneous breaking of supersymmetry to occur in a natural way. Thus the full supergravity effective potential of Eq. (60) differs from the global one of $V_{F}+V_{D}$, [Eqs. (16) and (17)] by not being positive definite. Hence Eq. (60) can easily accommodate supersymmetry breaking. A simple example of this is the choice [25] $\tilde{W}=m^{2}(z+B)$ (where $m^{2}$ and $B$ are constants) with a flat Kahler potential ( $d=\sum_{i} z_{i} z_{i}^{\dagger}$ ). Minimizing the potential of Eq. (60) yields the result

$$
\left\langle z>=\kappa^{-1} a(\sqrt{2}-\sqrt{6}) ; a= \pm 1\right.
$$


We see that $\langle z\rangle=O\left(M_{P \ell}\right)$ and so the Planck terms in $V$ are crucial to achieve this result. [One can also fine tune the cosmological constant to zero (arrange that $V_{\min }=0$ ) by chosing $B=-\kappa^{-1} a(2 \sqrt{2}-\sqrt{6})$.] The existance of a non-zero $\langle z\rangle$ is a signal of supersymmetry breaking via a "super-Higgs" effect. The chiral partner of $z(x)$ ie. $\chi_{z}(x)$ is absorbed by the gravitino $\psi^{\mu}(x)$ which then becomes massive with mass $m_{3 / 2}$. (The extra two helicities of $\chi_{z}$ then gives the $\psi^{\mu}(x)$ four fermionic helicity states, the correct number for a hermitian massive spin $3 / 2$ field. The complex Higgs field $z(x)$ has two bose degrees of freedom, which with the massless graviton $g_{\mu \nu}(x)$ gives four bosonic states.) From Eq. (66) one has

$$
m_{3 / 2}=\frac{1}{2} \kappa^{2} \mid\langle W(<z>)| e^{\frac{\pi^{2}}{4}\langle z\rangle^{2}}
$$

which shows that $m_{\mathrm{3} / 2} \approx \kappa m^{2}$. We will see below that supersymmetry breaking triggers $S U(2) \times U(1)$ breaking at the electroweak scale so that $\mathrm{\kappa m}^{2}=O\left(M_{Z}\right)$, which implies $m=O\left(10^{10} \mathrm{GeV}\right)$ for this case.

An alternate way of breaking supersymmetry is via a gaugino condensate [26], $m_{s}^{3} \equiv\left\langle\lambda \gamma^{0} \lambda\right\rangle \neq 0$. Then it turns out that $m_{3 / 2} \sim \kappa^{2}\left\langle\lambda \gamma^{0} \lambda\right\rangle$ which is of electroweak size for $m_{s}=O\left(10^{12-13} \mathrm{GeV}\right)$. Such an effect requires non-perturbative phenomena to occur, and hence it is difficult to make an explicit calculation in this mechanism for supersymmetry breaking.

\section{Supergravity Gut Models}

We consider in this section the construction of grand unified models based on supergravity [27]. The advantage of using local supersymmetry is that, as we have seen, spontaneous breaking of supersymmetry is easily achieved as a consequence of the supergravity interactions. However, a new hierarchy problem arises. Since the super Higgs VEV $\langle z\rangle=O\left(M_{P \ell}\right)$, there is a danger that the breaking of supergravity will communicate huge Planck size masses to the physical particles. The secret of preventing this is to allow the super Higgs field to couple only very weakly to the physical matter fields i.e. only gravitationally. This can be accomplished by requiring:

(i) The super Higgs field $z(x)$ be a gauge singlet.

(ii) $\tilde{W}\left(z_{i}\right)=W\left(z_{a}\right)+W_{h}(z)$ where $\left\{z_{a}\right\}$ are the physical fields.

Condition (i) guarentees that there are no gauge couplings between $z(x)$ and the physical fields $\left\{z_{a}(x)\right\}$, while from Eq. (60) one sees that the other couplings between $z(x)$ and $z_{a}(x)$ are always scaled by powers of $\kappa=1 / M_{P \ell}$ and hence gravitationally suppressed. Thus (i) and (ii) implies that supersymmetry breaking lives in a "hidden" sector, screened from the physical sector by gravity. (Such hidden sectors also exist in string theory.)

The discussion of the unification of the SM coupling constants suggests that the SM group is a valid symmetry below the Gut scale $M_{G}$. Thus as one proceeds from $M_{P \ell}$ down to $M_{G}$, one expects the Gut gauge group $G$ of the physical sector 
to break to the SM at $\mu=M_{G}: G \rightarrow S U(3)_{C} \times S U(2)_{L} \times U(1)_{Y}$. For the theory to be reasonable this must happen spontaneously with some of the fields, $\left\{z_{A}(x)\right\}$ growing VEVs and superheavy masses:

$$
\left\langle z_{A}>=O\left(M_{G}\right) ; M_{z_{A}}=O\left(M_{G}\right)\right.
$$

One may now integrate out the superheavy fields $z_{A}$ and eliminate the super Higgs fields to obtain an effective $S U(3) \times S U(2) \times U(1)$ theory just below $M_{G}$. A remarkable theorem then holds [28]:

Consider a class of models which obey the following conditions:

(i) There exists a "hidden" sector which is gauge singlet with respect to the physical sector gauge group $G$ which breaks supersymmetry (e.g. by a super Higgs or gaugino condensate). The hidden sector communicates only gravitationally with the physical sector.

(ii) There exists a Gut sector which breaks $G$ to the Standard Model at scale $Q=M_{G}: G \rightarrow S U(3)_{C} \times S U(2)_{L} \times U(1)_{Y}$.

(iii) After integrating out the superheavy fields and eliminating the super Higgs fields, the only light particles remaining below the Gut scale are those of the supersymmetrized Standard Model with one pair of light Higgs doublets.

(iv) Any super Higgs couplings that appear in the Kahler potential are generation independent.

Then, for models where the non-linear parts of $f_{\alpha \beta}$ and $d$ are scaled by $\kappa \equiv 1 / M_{P \ell}$, the renormalizable interactions below $M_{G}$ (which equivalently arise in the limit $\kappa \rightarrow 0$ ) are described by an effective superpotential with quadratic and cubic parts, $W=W^{(2)}+W^{(3)}$, and effective potential $V$,

$$
V=\left\{\sum_{a}\left|\frac{\partial W}{\partial z_{a}}\right|^{2}+V_{D}\right\}+\left\{m_{0}^{2} z_{a} z_{a}^{\dagger}+\left(A_{0} W^{(3)}+B_{0} W^{(2)}+\text { h.c. }\right),\right.
$$

and a universal gaugino mass term

$$
\mathcal{L}_{\text {mass }}^{\lambda}=-m_{1 / 2} \bar{\lambda}^{\alpha} \lambda^{\alpha}
$$

If the model is $R$ parity invariant or alternately lepton number conserving, then $W$ has the unique form (required by $S U(3) \times S U(2) \times U(1)$ invariance),

$$
W=\mu_{0} H_{1} H_{2}+\left[\lambda_{i j}^{(u)} q_{i} H_{2} u_{j}^{C}+\lambda_{i j}^{(d)} d_{i} H_{1} d_{j}^{C}+\lambda_{i j}^{(e)} l_{i} H_{1} d_{j}^{C}\right]
$$

where $\lambda_{i j}^{(u, d, e)}$ are the Yukawa coupling constants [analogous to those in Eq. (5)] and $H_{1,2}$ the two light Higgs doublets.

Assumptions (i) - (iv) lead to a wide class of models. Thus conditions (ii) and (iii) are what is needed to achieve grand unification [i.e. the SM group holds up to $M_{G}$ with the particle spectrum as stated in (iii)]. Condition (i) is what is 
required maintain the gauge hierarchy and (i) and (iv) guarantee the suppression of flavor changing neutral interactions. Thus it is difficult to remove any of these assumption and still maintain a phenomenologically viable model.

Eqs. (71) and (73) show that the effective theory below the Gut scale is a broken global SUSY theory ( $V_{D}$ is the usual $D$ term) with four soft breaking terms. These are scaled by the mass parameters $m_{0}$ (universal mass of the chiral multiplet spin zero fields), $m_{1 / 2}$ (universal gaugino mass), $A_{0}$ and $B_{0}$ (scaling factor of cubic and quadratic parts of the superpotential). These four constants are determined by the nature of the hidden sector, i.e. they parameterize our ignorance of the hidden sector where supersymmetry breaks (e.g. by a hidden sector superpotential $W_{h}(z)$ or by hidden sector gaugino condensates). In addition there is a Higgs mixing parameter $\mu_{0}$.

It is interesting to compare the simplicity of this result to global SUSY theory. There one added in soft breaking terms by hand, Eqs. (24) and (25), and the most general form depended on 137 parameters. Effectively the supergravity theory determines 133 of these parameters!

We briefly indicate how the various soft breaking terms in Eqs. (71), (72) arise for the case of super Higgs breaking of supersymmetry. Thus from Eq. (60), the term $\left(d^{-1}\right)_{j}^{j}\left[\kappa^{2} d, \tilde{W}\right] \quad\left[\kappa^{2} d_{j} \tilde{W}\right]^{\dagger}$ gives rise to $\left(\kappa^{2}|\tilde{W}|^{2}\right) z_{i} z_{i}^{\dagger}$ and hence to $m_{0}^{2} \sim$ $\left(\kappa^{2}<W_{h}>\right)^{2}$. Similarly, cross terms in Eq. (60) such as $\left(d^{-1}\right)_{j}^{i} \kappa^{2} d_{i} \tilde{W}\left(\partial \tilde{W} / \partial z_{j}\right)$ give rise to $z_{i}\left(\partial W^{(2,3)} / \partial z_{i}\right) \kappa^{2}\left\langle W_{h}>\right.$ which yield $A_{0}$ and $B_{0}$ type structures. If $f_{\alpha \beta}=$ $\delta_{\alpha \beta}(1+c \kappa z+\cdots)$ then from Eq. (65), for $i=j=z$, the VEV of the bracket gives a term of form $\kappa^{3}<z W_{h}>$ and since $\left\langle z>\sim \kappa^{-1}\right.$ one has $m_{1 / 2} \sim \kappa^{2}\left\langle W_{h}\right\rangle$. Finally we note that the Kahler potential can have a form $d=z_{i} z_{i}^{\dagger}+f\left(z_{i}\right)+f^{\dagger}\left(z_{i}\right)$ and the most general gauge invariant and lepton number invariant quadratic piece for $f$ is $f\left(z_{i}\right)=\mathrm{cH}_{1} H_{2}$ where $c$ is dimensionless. (Higher terms in $f\left(z_{i}\right)$ load to non-renormalizable interactions scaled by $\kappa=1 / M_{P \ell}$.) By the Kahler transformation of Eq. (56) this term can be moved into the superpotential yielding $W e^{\kappa^{2} s}=W+c \kappa^{2} H_{1} H_{2} W+\cdots$ and hence a quadratic term $c \kappa^{2}\left\langle W_{h}\right\rangle H_{1} H_{2}$ showing that $\mu_{0} \sim \kappa^{2}\left\langle W_{h}\right\rangle$. Thus we see that all the parameters $m_{0}, m_{1 / 2}, A_{0}, B_{0}$ and $\mu_{0}$ are of the same general size, i.e. $\kappa^{2}\left\langle W_{h}\right\rangle$, and we will see later that this mass is the electroweak mass scale.

\section{An Example: $S U(5)$ Supergravity Gut Model}

In this section we discuss an explicit example of a supergravity Gut model based on the Gut group $G=S U(5)$. Here each generation of quarks and leptons are in the $10+\overline{5}$ representations of $S U(5)$ :

$$
10=M_{i}^{X Y}=-M_{i}^{Y X} ; \overline{5}=\bar{M}_{i Y} ; i=1,2,3 X, Y=1 \ldots 5
$$

where $i$ is the generation index and $X, Y$ are group indices. In addition, the Higgs are in $5+\overline{5}$ representations

$$
5=H_{1}^{X} ; \overline{5}=\bar{H}_{2 X}
$$


Thus if we decompose the $S U(5)$ labels into their $S U(3) \times S U(2)$ content, $X=(a, \alpha) a=$ $1 \ldots 3, \alpha=4,5$ then $H_{1}^{\alpha}$ and $\bar{H}_{2 \alpha}$ are the $H_{1}, H_{2}$ doublets of the Standard Model, and in addition there are two color triplets $(3$ and $\overline{3})$ Higgs $H_{1}^{a}, \bar{H}_{2 a}$.

The total superpotential has a Yukawa, a Gut and a hidden part: $\bar{W}=$ $W_{Y}+W_{G}+W_{h} . W_{h}$ is chosen to break supersymmetry. The $S U(5)$ invariant Yukawa interactions are

$$
W_{Y}=\lambda_{i j}^{1} \varepsilon_{X Y Z W U} H_{1}^{X} M_{i}^{Y Z} M_{j}^{W U}+\lambda_{i j}^{2} \bar{H}_{2 X} \bar{M}_{i Y} M_{j}^{X Y}
$$

In writing Eq. (76) we have assumed invariance under the discrete symmetry (matter parity) of $\left(M_{i}^{X Y}, \bar{M}_{i X}\right) \rightarrow\left(-M_{i}^{X Y},-\bar{M}_{i X}\right),\left(H_{1}^{X}, \bar{H}_{2 X}\right) \rightarrow\left(H_{1}^{X}, \bar{H}_{2 X}\right)$ to forbid the cubic interactions $\bar{M}_{i X} \bar{M}_{j Y} M_{k}^{X Y}$ which would give rise to too rapid proton decay. The Gut sector (originally proposed in global SUSY models [29]) uses a 24 representation of $S U(5), \Sigma_{Y}^{X}$, to break $S U(5)$ to the SM. The form of $W_{G}$ is

$$
\begin{aligned}
W_{G}= & \lambda_{1}\left[\frac{1}{3} \operatorname{Tr} \Sigma^{3}+\frac{1}{2} M T r \Sigma^{2}\right. \\
& +\lambda_{2} \bar{H}_{2 X}\left[\Sigma_{Y}^{X}+2 M^{\prime} \delta_{Y}^{X}\right] H_{1}^{Y}
\end{aligned}
$$

where $T r$ is the trace over $S U(5)$ indices, and $M, M^{\prime}$ are mass parameters of $O\left(M_{G}\right)$. In the following we will set $M^{\prime}=M$.

One may now minimize the effective potential. $W_{h}$ causes the breaking of supersymmetry and gives rise to the soft breaking parameters of Eq. (73). Assuming these are much smaller than $M_{G}$, one finds upon minimizing the effective potential of Eq. (71) that $\Sigma_{Y}^{X}$ grows a VEV which to leading order is

$$
\operatorname{diag}<\Sigma_{Y}^{X}>=M(2,2,2,-3,-3)\left[1+O\left(\frac{m_{S B}}{M}\right)\right]
$$

where $m_{S B}$ is any of the soft breaking parameters $m_{0}, m_{1 / 2}$ etc. (Since we will see that $m_{S B}=O\left(M_{Z}\right)$, characteristically $O\left(m_{S B} / M\right) \approx 10^{-14}$ and hence negligible. Eq. (78) clearly breaks $S U(5)$ and preserves $S U(3) \times S U(2) \times U(1)$. The $\Sigma_{Y}^{X}, H_{1}^{a}$ and $H_{2 a}$ become superheavy with masses $O(M) \approx M_{G}$ while the $S U(2)$ doublets $H_{1}^{\alpha}, \bar{H}_{2 \alpha}$ remain light with masses $O\left(m_{S B}\right)$ and $O\left(\mu_{0}\right)$. Integrating out the superheavy fields in $W_{Y}$ and $W_{G}$ and eliminating the super Higgs (which effectively means replacing it by its VEV) one finds that the renormalizable interactions are governed by an effective superpotential which is precisely the form of Eq. (71) [with effective potential and gaugino mass term of Eqs. (73) and (72)]. Thus at the Gut scale, the theory has precisely the form of the MSSM with $H_{1}=H_{1}^{\alpha}$ and $H_{2}=\bar{H}_{2 \alpha}$ playing the role of the two light Higgs doublets but with four soft breaking terms and a Higgs mixing parameterized by

$$
m_{0}, m_{1 / 2}, A_{0}, B_{0} \text { and } \mu_{0}
$$

There is one theoretically unpleasant aspect about the above model. In Eq. (77) we set $M^{\prime}=M$, and it is this that kept the Higgs doublets light (i.e. the doublet masses are proportional to $M^{\prime}-M$.) While the no-renormalization 
theorem of supersymmetry guarantees that this condition is maintained even with loop corrections, this is indeed an awkward fine tuning. There are several ways of avoiding this problem:

\section{(i) Missing Partner Mechanism}

By using the 75, 50 and $\overline{50}$ representations in $W_{G}$ to break $S U(5)$, instead of the 24, one can prevent the Higgs doublets from growing masses [30]. Recall that

$$
\begin{aligned}
& 75=\Sigma_{U W}^{X Y}, \Sigma_{U Y}^{X Y} \equiv 0 ; 50=\theta_{X Y Z}^{U W}, \Theta_{X Y W}^{U W} \equiv 0 \\
& \overline{50}=\bar{\Theta}_{U W}^{X Y Z}, \bar{\Theta}_{U W}^{X Y W} \equiv 0
\end{aligned}
$$

where subscripted indices are all anti-symmetric as are superscripted indices. Instead of Eq. (77) one can chose

$$
\begin{aligned}
W_{G}= & \lambda_{1} \Theta_{X Y Z}^{U W} \Sigma_{U W W}^{X Y} H_{1}^{Z}+\lambda_{2} \bar{\Theta}_{U W}^{X Y Z} \Sigma_{X Y}^{U W} \bar{H}_{2 Z} \\
& +M \Theta_{X Y Z}^{U W} \bar{\Theta}_{U W}^{X Y Z}+f(\Sigma)
\end{aligned}
$$

where $f(\Sigma)$ is chosen so that $\Sigma_{U W}^{X Y}$ grows a non-vanishing VEV of $O(M)$. Now the $S U(3)_{C} \times S U(2)_{L}$ content of the 50 is

$$
50=(8,2)+(6,3)+(6,1)+(3,2)+(\overline{3}, 1)+(1,1)
$$

i.e. there is no doublet $(1,2)$ term. Thus when $\Sigma$ is replaced by its VEV in Eq. (81) there is no piece of $\Theta$ to match up to form a mass term with the $H_{1}^{\alpha}$ doublet in the $\lambda_{1}$ term (and similarly no piece of $\bar{\theta}$ can form a mass term with the $\bar{H}_{2 \alpha}$ doublet in the $\lambda_{2}$ term). Note that the 50 does contain a $(3,1)$ piece and the $\overline{50}$ contains a $(3,1)$ piece and so the color triplets, $H_{1}^{a}, \bar{H}_{2 a}$ do indeed become superheavy.

\section{(ii) Higgs Doublets as Goldstone Bosons}

An alternate procedure is to choose a form for $W_{G}$ with a larger global symmetry which has the gauge group $G$ as a subgroup [31]. [For example, in the case $G=S U(5)$, one may give $W_{G}$ a global $S U(6)$ symmetry by extending $\Sigma_{Y}^{X} X, Y=1 \cdots 6$ to be a 35 of $S U(6)$ and similarly $H_{1}^{X}, \bar{H}_{2 X}$ be 6 and 6 of $S U(6)$.] When the local $S U(5)$ breaks spontaneously, the global $S U(6)$ also breaks. The Higgs color triplets become superheavy as before, but the Higgs doublets are the Goldstone bosons arising from the breaking of the global $S U(6)$. Thus they remain massless. One in fact finds in this analysis that $M=M^{\prime}$ occurs automatically, which illustrates that one man's fine tuning may be another man's group symmetry!

(iii) Flipped $S U(5) \times U(1)$

A third method of keeping the Higgs doublets light is to change the group symmetry to $S U(5) \times U(1)$ and flipping the embedding of the particle spectrum [32]. Thus one uses a $10+\overline{5}+1$ of $S U(5)$ for each generation, but interchanges $u$ and $d$ quarks and $e$ and $\nu$ leptons, the $e^{c}$ field then appears in the singlet representation with a right handed neutrino replacing it in the 10 representation. Instead of using 
a 24 to break $S U(5)$, one introduces 10 and $\overline{10}$ fields along with the usual $H_{1}$ and $\bar{H}_{2}$. The Gut Yukawa couplings are then $\lambda_{1} 1010 H_{1}+\lambda_{2} \overline{10} \overline{10} \bar{H}_{2}$. The $S U(3)_{C} \times S U(2)_{L}$ content of a 10 representation is $(\overline{3}, 1)+(3,2)+(1,1)$ and so when the 10 grows a VEV one has a natural missing partner for the doublets (e.g. no $(1,2)$ piece in the 10 to form a mass term with the $\left.H_{1 \alpha}\right)$. However, there is a $(3,1)$ which combines with the $H_{1}^{a}$ to make the color triplets superheavy. The drawback of this model is that it is not fully unified since $G$ is a product group. A possible solution to this difficulty is discussed in Sec. 17.

\section{Radiative Breaking of $S U(2) \times U(1)$}

In the $S M$, the spontaneous breaking of $S U(2) \times U(1)$ is arranged by hand by inserting in a negative (mass) ${ }^{2}$ in the Higgs potential. Thus the SM accomodates the breaking of $S U(2) \times U(1)$ but does not deduce it's existance from any of the basic principles of the theory. One of the remarkable features of supergravity grand unification is that it offers a natural explanation of $S U(2) \times U(1)$ breaking arising from radiative corrections which generate dynamically the required negative (mass) ${ }^{2}$ [33]. Further, it is the spontaneous breaking of supersymmetry at the Planck scale that triggers the breaking of $S U(2) \times U(1)$ at the electroweak scale, a result that is a consequence of supergravity theory [34]. Hence the two spontaneous breakings are unified.

We consider here the general supergravity model which is described at the Gut scale by Eqs. (71)-(73). Aside from the Yukawa coupling constants (which are the same as in the SM) this theory depends upon seven constants: $m_{1 / 2}, m_{0}, A_{0}, B_{0}$; $\mu_{0} ; \alpha_{G}, M_{G}$. This might be compared with the SM which depends upon $m^{2}, \lambda$ (from the Higgs potential); $\alpha_{1}, \alpha_{2}, \alpha_{3}$. Thus one needs only two more parameters in supergravity Gut models than in the SM. (Also, $\alpha_{G}$ and $M_{G}$ may be viewed as having been "measured" by the unification analysis of Sec. 6.) As we will see, radiative breaking of $S U(2) \times U(1)$ will allow us to eliminate one more parameter, and so the general model depends on relatively few parameters and therefore should have significant predictive power.

The Gut theory is initially defined at $M_{G}$, while experiment takes place at the electroweak scale. One may use the Renormalization Group Equations (RGE) to connect these two domains. For discussion of electroweak breaking we need the part of the effective potential involving the Higgs fields $H_{1}$ and $H_{2}$. The renormalization group improved Higgs potential is $V_{H}=V_{0}+\Delta V_{1}$ where $V_{0}$ is the tree part [which can be read off Eqs. (71) and (73)] and $\Delta V_{1}$ is the one loop correction. One finds

$$
\begin{array}{r}
V_{0}=m_{1}^{2}\left|H_{1}\right|^{2}+m_{2}^{2}\left|H_{2}\right|^{2}-m_{3}^{2}\left(H_{1} H_{2}+\text { h.c. }\right) \\
+\frac{1}{8}\left(g_{2}^{2}+g_{Y}^{2}\right)\left(\left|H_{1}\right|^{2}-\left|H_{2}\right|^{2}\right)^{2}
\end{array}
$$

and [35] 


$$
\Delta V_{1}=\frac{1}{64 \pi^{2}} \sum_{a}(-1)^{2 \varepsilon_{a}} n_{a} M_{a}^{4} \ln \left[\frac{M_{a}^{2}}{e^{3 / 2} Q^{2}}\right]
$$

All parameters, $m_{i}(t), g_{2}(t), g_{Y}(t)$ are "running" parameters at scale $Q$ where $t=$ $\ln \left[M_{G}^{2} / Q^{2}\right]$. Thus

$$
\begin{aligned}
& m_{i}^{2}(t)=m_{H_{i}}^{2}(t)+\mu^{2}(t) ; i=1,2 \\
& m_{3}^{2}(t)=-B(t) \mu(t)
\end{aligned}
$$

with boundary conditions at $Q=M_{G}(t=0)$ :

$$
\begin{aligned}
m_{i}^{2}(0) & =m_{0}^{2}+\mu_{0}^{2}, i=1,2 ; m_{3}^{2}(0)=-B_{0} \mu_{0} \\
\alpha_{2}(0) & =\alpha_{G}=(5 / 3) \alpha_{Y}(0)
\end{aligned}
$$

In Eq. (84), $M_{a}=M_{a}\left(v_{1}, v_{2}\right)$ is the tree level mass of particle $a$ as function of the VEVs $v_{i}=\left\langle H_{i}\right\rangle$, and $s_{a}$ and $n_{a}$ are the spin and number of helicity states of particle $a$.

The RGE are given at the end of this section. They allow one to express all the parameters in Eqs. $(83,84)$ in terms of the Gut scale parameters. The procedure one uses is as follows: A specific model corresponds to a choice of Gut scale parameters $m_{0}, m_{1 / 2}, A_{0}, B_{0}$, and $\mu_{0}$. At the Gut scale, all scalar particles have an initial positive (mass) ${ }^{2}$ of $m_{0}^{2}$. One then integrates down in energy until a (mass) ${ }^{2}$ turns negative, signaling the breakdown of $S U(2) \times U(1)$ and a VEV growth $v_{i}=<H_{i}>\neq 0$. Let us first look at the tree potential $V_{0}$. Here $S U(2) \times U(1)$ breaking implies that the determinant of the (mass) ${ }^{2}$ matrix be negative ( 80 that one negative (mass) ${ }^{2}$ eigenvalue exists) i.e. from Eq. (83),

$$
\mathcal{D} \equiv m_{1}^{2} m_{2}^{2}-m_{3}^{4}<0
$$

Also, for a valid minimum, one requires that the potential be bounded from below, which is satisfied if

$$
\mathcal{L} \equiv m_{1}^{2}+m_{2}^{2}-2\left|m_{3}^{2}\right|>0
$$

Turning now to the full potential, the minimization conditions, $\partial V_{H} / \partial v_{i}=0$ yields the relations

$$
\frac{1}{2} M_{Z}^{2}=\frac{\mu_{1}^{2}-\mu_{2}^{2} \tan ^{2} \beta}{\tan ^{2} \beta-1} ; \sin 2 \beta=\frac{2 m_{3}^{2}}{\mu_{1}^{2}+\mu_{2}^{2}}
$$

where $\tan \beta \equiv v_{2} / v_{1}, \mu_{i}^{2}=m_{1}^{2}+\Sigma_{i}$ and $\Sigma_{i}$ is the loop correction

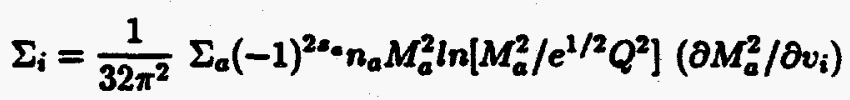

Since $|\sin 2 \beta| \leq 1$, the second equation of (89) includes a generalization of the stability condition Eq. (88) with loop corrections. 
Normally, one would use minimization of $V_{H}$ to solve for the two VEVs $v_{i}=\left\langle H_{i}\right\rangle$. Instead, it is more convenient to use them to eliminate two of the Gut parameters in terms of $\tan \beta$ and the other parameters. A convenient choice is to use the second equation of (89) to eliminate $B_{0}$ and the first equation to eliminate $\mu_{0}$. Since only $\mu^{2}$ enters into the first equation, there are two branches depending on the sign of $\mu$ i.e. $\mu>0$ and $\mu<0$. One then has that the low energy physics depends only on four parameters:

$$
m_{0}, m_{1 / 2}, A_{0}, \tan \beta=v_{2} / v_{1}
$$

Thus the 32 SUSY particle masses can be determined in terms of these four parameters (and the as yet unknown $t$-quark mass $m_{t}$ ).

There are several fine points worth mentioning:

(i) If we define $Q_{0}$ as the mass scale where $\mathcal{D}(Q)$ of Eq. (87) vanishes and $Q_{1}$ the point where $\mathcal{L}(Q)$ vanishes, in general one finds that $Q_{0}>Q_{1}$. Thus at the tree level, satisfactory electroweak breaking occurs when $Q_{1}<Q<Q_{0}$. In running the RGE from $M_{G}$ down to $Q$, what value should we choose for $Q$ ? (Where should we stop the RG "clock"?) It has been shown that when the loop correction $\Delta V_{1}$ is included, the results are approximately independent of $Q[36]$. In the following we will set $Q=M_{Z}$. This is convenient since then $M_{Z}\left(Q=M_{Z}\right)$ is the physical (experimental) $Z$ boson mass. [All other masses then are running masses at scale $Q=M_{Z}$ (and can differ from their physical values)]. Actually, matters are simpler. In most of the parameter space, there is a large amount of cancelation in $\Sigma_{i}$ and so the one loop correction is small [37]. Thus using $V_{0}$ alone generally gives a good approximation, and the $Q$ dependence of results are small.

(ii) In the analysis given above, we have tacitly assumed that it is a Higgs (mass) ${ }^{2}$ that turns negative as one runs the RGE from $M_{G}$ to the electroweak scale. It is possible that some other (mass) ${ }^{2}$ might turn negative first e.g. a squark or slepton. This would lead to a catastrophe that $S U(3)_{C}$ or electromagnetism breaks down. A number of necessary conditions for this not to happen have been established [38] (though sufficiency conditions are not known). Throughout most of the parameter space, the known conditions are satisfied, and it is $m_{\mathrm{H}_{3}}^{2}$ which turns negative. The characteristic situation is shown schematically in Fig. 7.

The existance of solutions to Eqs. (89) is the necessary conditions that electroweak breaking actually occurs. No satisfactory solutions will exist, however, unless three requirements are met:

1. At least one of the soft breaking parameters $m_{0}, m_{1 / 2}, A_{0}, B_{0}$ are non-zero

2. $\mu_{0}$ is non-zero

3. $m_{t}$ is heavy $\left(m_{t} \gtrsim 90 \mathrm{GeV}\right)$ 


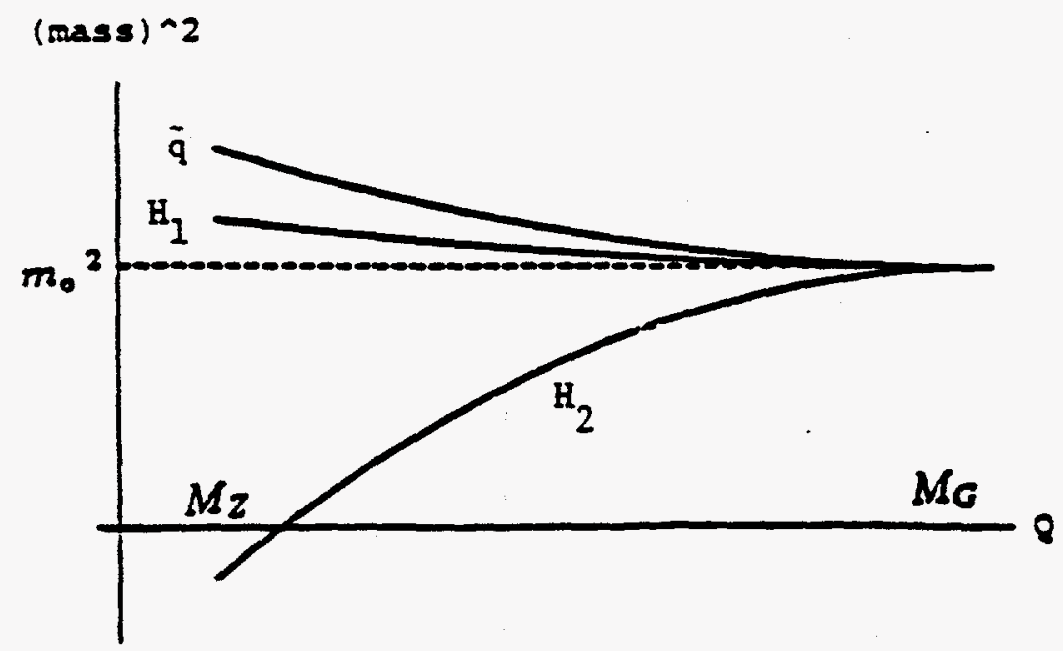

Figure 7: Schematic diagram of running of masses from $M_{G}$ to electroweak scale. The heavy top quark bends $m_{H_{2}}^{2}$ downwards as $Q$ decreases.

Condition (1) shows in a real sense that the breaking of supersymmetry at the Gut scale generates $S U(2) \times U(1)$ breaking at the electroweak scale for if all the soft breaking parameters generated by supergravity vanished, there would be no electroweak breaking. Similarly $\mu_{0}$ (which is also generated from supergravity interactions) must be non-zero and $m_{t}$ must be heavy since it is the $t$-quark Yukawa coupling to $H_{2}$ that drives $m_{H,}^{2}$ negative. Thus there is a confluence of three items, two of them theoretical aspects of supergravity, and one of them experimental that gives rise to electroweak breaking. The fact that the top quark must be heavy was one of the first predictions of supergravity Guts [33].

\section{Renormalization Group Equations}

We list here for reference the one loop RGE. For simplicity, we keep only the large $t$-quark Yukawa couplings, which is a good approximation for $\tan \beta<10$. Our notation follows mainly that of Ibañez et al [33] (except that $\alpha_{1} \equiv(5 / 3) \alpha_{Y}$ ). Unfortunately, a number of different sign conventions for $t, A_{t}$ and $\mu$ exist in the literature. We use $t=\ln \left(M_{\mathrm{G}}^{2} / Q^{2}\right)$.

(i) Gauge couplings:

$$
\frac{d \tilde{\alpha}_{i}}{d t}=-\frac{b_{i}}{4 \pi} \tilde{\alpha}_{0}^{2} ; b_{i}=(33 / 5,1,-3), \tilde{\alpha}_{i}=\alpha_{i} / 4 \pi
$$

with boundary conditions $\alpha_{i}(0)=\alpha_{G}$.

(ii) Gaugino masses:

$$
\frac{d \tilde{m}_{i}}{d t}=-\frac{b_{i}}{4 \pi} \tilde{\alpha}_{i}(t) \tilde{m}_{i}(t) ; \tilde{m}_{i}(0)=m_{1 / 2}
$$


(iii) Higgs masses:

$$
\begin{gathered}
\frac{d m_{H_{1}}^{2}}{d t}=3 \tilde{\alpha}_{2} \tilde{m}_{2}^{2}+(3 / 5) \tilde{\alpha}_{1} \tilde{m}_{1}^{2} \\
\frac{d m_{H_{2}}^{2}=}{d t}= \\
\\
\left.\quad 3 \tilde{\alpha}_{2} \tilde{m}_{2}^{2}+(3 / 5) \tilde{\alpha}_{1} \tilde{m}_{1}^{2}\right) \\
-3 Y_{t}\left(m_{Q}^{2}+m_{U}^{2}+m_{H_{2}}^{2}+A_{\imath}^{2}\right)
\end{gathered}
$$

with boundary conditions $m_{H_{i}}(0)=m_{0}$. (Note that it is the $t$-quark Yukawa couplings, $Y_{t}$, which causes $m_{H_{2}}^{2}$ to decrease as $t$ increases.)

(iv) t-quark Yukawa coupling:

$$
\frac{d Y_{t}}{d t}=\left(\frac{16}{3} \tilde{\alpha}_{3}+3 \tilde{\alpha}_{2}+\frac{13}{15} \tilde{\alpha}_{1}-6 Y_{t}\right) Y_{t}^{2}
$$

where $Y_{t}=\lambda_{l}^{2} / 4 \pi$ and $\lambda_{t}(Q)$ is the $t$-quark Yukawa coupling constant defined by $m_{t}=\lambda_{t}\left(m_{t}\right)<H_{2}>$.

(iv) $t$-quark $A$ parameter:

$$
\left.\frac{d A_{t}}{d t}=(16 / 3) \tilde{\alpha}_{3} \tilde{m}_{3}+3 \tilde{\alpha}_{2} \tilde{m}_{2}+(13 / 15) \tilde{\alpha}_{1} \tilde{m}_{1}\right)-6 Y_{t} A_{t}
$$

where $A_{t}(0)=A_{0}$.

(v) $B$ parameter:

$$
\frac{d B}{d t}=\left(3 \tilde{\alpha}_{2} \tilde{m}_{2}+(3 / 5) \tilde{\alpha}_{1} \tilde{m}_{1}\right)-3 Y_{t} A_{t}
$$

where $B(0)=B_{0}$.

(vi) $\mu$ parameter:

$$
\frac{d \mu^{2}}{d t}=\left(3 \tilde{\alpha}_{2}+(3 / 5) \tilde{\alpha}_{1}-3 Y_{t}\right) \mu^{2}
$$

where $\mu(0)=\mu_{0}$. (Note that the sign of $\mu_{0}$ is undetermined by the $\mu$ RGE.)

(vii) Squark masses $(i=1,2)$ :

$$
\begin{aligned}
& Q_{i} \equiv\left(\tilde{u}_{i L}, \tilde{d}_{i L}\right) ; U_{i} \equiv \tilde{u}_{R i}, D_{i} \equiv \tilde{d}_{R i} \\
& \frac{d m_{Q_{i}}^{2}}{d t}=(16 / 3) \tilde{\alpha}_{3} \tilde{m}_{3}^{2}+3 \tilde{\alpha}_{2} \tilde{m}_{2}^{2}+(1 / 15) \tilde{\alpha}_{1} \tilde{m}_{1}^{2} \\
& \frac{d m_{U_{i}}^{2}}{d t}=(16 / 3) \tilde{\alpha}_{3} \tilde{m}_{3}^{2}+(16 / 15) \tilde{\alpha}_{1} \tilde{m}_{1}^{2}
\end{aligned}
$$




$$
\left.\frac{d m_{D_{i}}^{2}}{d t}=(16 / 3) \tilde{\alpha}_{3} \tilde{m}_{3}^{2}+(4 / 15) \tilde{\alpha}_{1} \tilde{m}_{1}^{2}\right)
$$

where $m_{Q_{i}}(0)=m_{U_{i}}(0)=m_{D_{i}}(0)=m_{0}$.

(viii) Slepton masses $(i=1,2,3)$

$$
\begin{array}{r}
L_{i} \equiv\left(\tilde{\nu}_{L i}, \tilde{e}_{L i}\right) ; \tilde{E}_{i} \equiv \tilde{e}_{R i} \\
\frac{d m_{L i}^{2}}{d t}=3 \alpha_{2} \tilde{m}_{2}^{2}+(3 / 5) \tilde{\alpha}_{1} \tilde{m}_{1}^{2} \\
\frac{d m_{E i}}{d t}=(12 / 5) \tilde{\alpha}_{1} \tilde{m}_{1}^{2}
\end{array}
$$

where $m_{L_{i}}(0)=m_{E i}(0)=m_{0}$. (Note that the slepton and first two generations of squark (mass) ${ }^{2}$ all increase with increasing $t$.

(ix) Squark masses $(i=3)$

$$
\begin{aligned}
Q & \equiv\left(\tilde{u}_{3 L}, \tilde{d}_{3 L}\right) ; U \equiv \tilde{u}_{3 R}, D \equiv \tilde{d}_{3 R} \\
\frac{d m_{Q}^{2}}{d t}= & (16 / 3) \tilde{\alpha}_{3} \tilde{m}_{3}^{2}+3 \tilde{\alpha}_{2} \tilde{m}_{2}^{2}+(1 / 15) \tilde{\alpha}_{1} \tilde{m}_{1}^{2} \\
& -Y_{t}\left(m_{H_{2}}^{2}+m_{Q}^{2}+m_{U}^{2}+A_{t}^{2}\right) \\
\frac{d m_{U}^{2}}{d t} & =(16 / 3) \tilde{\alpha}_{3} \tilde{m}_{3}^{2}+(16 / 15) \tilde{\alpha}_{1} \tilde{m}_{1}^{2} \\
& -2 Y_{t}\left(m_{H_{3}}^{2}+m_{Q}^{2}+m_{U}^{2}+A_{t}^{2}\right) \\
\frac{d m_{D}^{2}}{d t} & \left.=(16 / 3) \tilde{\alpha}_{3} \tilde{m}_{3}^{2}+(4 / 15) \tilde{\alpha}_{1} \tilde{m}_{1}^{2}\right)
\end{aligned}
$$

where $m_{Q}(0)=m_{U}(0)=m_{D}(0)=m_{0}$.

The procedure of solving these equations is the following: (1) Solve (92) for $\alpha_{i}$ and insert into (93) to solve for $\tilde{m}_{i}$. (2) One can then integrate (94), (100)-(102) for $m_{H_{1}}^{2},\left(m_{Q_{i}}^{2}, m_{U_{i}}^{2}, m_{D_{i}}^{2}, i=1,2\right),(103)$ and $(104)$ for $\left(m_{L i}^{2}, m_{E i}^{2}, i=1,2,3\right)$ and (107) and (96) for $m_{D}^{2}$ and $Y_{t}$. (3) Using $Y_{t}$ one can solve (97) and (99) for $A_{t}$ and $\mu^{2}$ and using then using $Y_{t}$ and $A_{t}$ one determines $B$ from (98). (4) The three remaining equations, (95), (105), and (106) are coupled and must be solved simultaneously.

\section{Expressions for SUSY Masses}

The SUSY masses are quantities that are defined at the low energy electroweak scale and are experimentally accessible to current and future accelerators. 
Using the RGE one can express each SUSY mass in terms of the $4+1$ parameters which define the Gut model: $m_{0}, m_{1 / 2}, A_{0}, \tan , \beta$ and $m_{t}$. In this section, we discuss the explicit formulae for each SUSY mass.

(i) Gauginos

From Eqs. (92), (93) one finds $\tilde{m}_{i}(t)=\left[\alpha_{i}(t) / \alpha_{G}\right] m_{1 / 2}$ where $\alpha_{i}(t)=\alpha_{G} /(1+$ $\left.\beta_{i} t\right)$ and $\beta_{i}=b_{i} \alpha_{G} / 4 \pi$. These relations then imply

$$
\tilde{m}_{1}: \tilde{m}_{2}: \tilde{m}_{3}=\alpha_{1}: \alpha_{2}: \alpha_{3}
$$

a relation that is postulated in the MSSM.

(ii) Squarks ( $i=1,2)$; Sleptons $(i=1,2,3)$

The squark and slepton masses can be obtained from the mass matrix $m_{a b}^{2}=$ $\left\langle\partial^{2} V^{e f f} / \partial z_{a} \partial z_{b}^{\dagger}\right\rangle$ where $V^{e f f}$ is the low energy renormalization group improved effective potential derived from Eq. (71). Using Eqs. (100)-(104) to relate the low energy mass parameters to the Gut scale parameters for the first two generations of squarks and all the sleptons, one finds

$$
\begin{aligned}
m_{\tilde{u}_{i L}}^{2}=m_{0}^{2}+m_{u_{i}}^{2} & +\tilde{\alpha}_{G}\left[(8 / 3) f_{3}+(3 / 2) f_{2}+(1 / 30) f_{1}\right] m_{1 / 2}^{2} \\
& +\left(\frac{1}{2}-\frac{2}{3} \sin ^{2} \theta_{W}\right) M_{Z}^{2} \cos ^{2} \beta \\
m_{\tilde{d}_{i L}}^{2}=m_{0}^{2}+m_{d_{i}}^{2}+ & \tilde{\alpha}_{G}\left[(8 / 3) f_{3}+(3 / 2) f_{2}+(1 / 30) f_{1}\right] m_{1 / 2}^{2} \\
& +\left(-\frac{1}{2}+\frac{1}{3} \sin ^{2} \theta_{W}\right) M_{Z}^{2} \cos 2 \beta
\end{aligned}
$$

where $m_{u_{i}}, m_{d_{i}} i=1,2$ are quark masses, $\tilde{\alpha}_{G}=\alpha_{G} / 4 \pi$ and $f_{k}(t)=t\left(2-\beta_{k} t\right) /$ $\left(1+\beta_{k} t\right)^{2}, \beta_{k}=(33 / 5,1,-3)$. For the sleptons one similarly has

$$
\begin{gathered}
m_{\tilde{e}_{i L}}^{2}=m_{0}^{2}+m_{e i}^{2}+\tilde{\alpha}_{G}\left[(3 / 2) f_{2}+(3 / 10) f_{1}\right] m_{1 / 2}^{2} \\
+\left(-\frac{1}{2}+\sin ^{2} \theta_{W}\right) M_{Z}^{2} \cos 2 \beta \\
m_{\bar{e}_{i R}}^{2}=m_{0}^{2}+m_{e_{i}}^{2}+\tilde{\alpha}_{G}(6 / 5) f_{1} m_{1 / 2}^{2}-\sin ^{2} \theta_{W} M_{Z}^{2} \cos 2 \beta
\end{gathered}
$$




$$
m_{\tilde{\nu}_{i L}}^{2}=m_{0}^{2}+\tilde{\alpha}_{G}\left[(3 / 2) f_{2}+(3 / 10) f_{1}\right] m_{1 / 2}^{2}+(1 / 2) M_{Z}^{2} \cos 2 \beta
$$

where $m_{e i}, i=1,2,3$ are the charged lepton masses. The quark and lepton mass terms arise from the Yukawa coupling terms of the superpotential Eq. (73).

If $m_{0}$ is large, the squarks and sleptons are nearly degenerate, and even if $m_{0}=0$ (as in the No-scale models), the squarks are still approximately degenerate since the $f_{3}$ term dominates. This is indeed what is assumed in the MSSM. If $m_{0}$ is small, however, one would not expect the sleptons to be degenerate.

One of the remarkable features of Eqs. $(109,111)$ is that

$$
m_{\tilde{c}}^{2}-m_{\tilde{u}}^{2}=m_{c}^{2}-m_{u}^{2}
$$
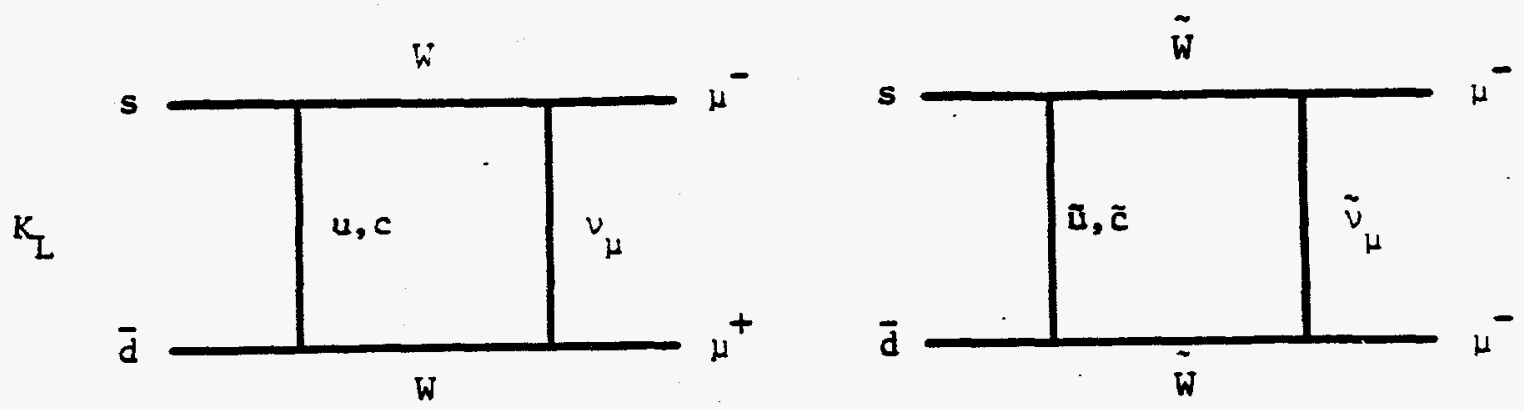

Figure 8: Contributions to $K_{L} \rightarrow \mu^{+} \mu^{-}$. The left diagram (the usual GMM term) is of size ( $m_{c}^{2}-$ $\left.m_{u}^{2}\right) / M_{W}^{2}$ while the right diagram (the additional SUSY term) is of size $\left(m_{\tilde{c}}^{2}-m_{\tilde{u}}^{2}\right) / M_{\bar{W}}^{2}$ or $\left(m_{\tilde{c}}^{2}-\right.$ $\left.\boldsymbol{m}_{\tilde{\mathbf{u}}}^{2}\right)\left(\boldsymbol{m}_{\tilde{\mathbf{q}}}^{2}\right)$.

Condition (116) guarentees a super GIM mechanism for suppressing flavor changing neutral interactions. Fig. 8 shows this phenomena for the case of $K_{L} \rightarrow \mu^{+} \mu^{-}$(first discussed in global SUSY models by Dimopoulos and Georgi [29]). Were it not for Eq. (116), the Wino contribution to $K_{L} \rightarrow \mu^{+} \mu^{-}$would be very large since $m_{\tilde{q}} \gtrsim$ $100 \mathrm{GeV}$. Thus there is an enormous cancelation in the squark mass differences. This cancelation can be traced to the fact that at the Gut scale, each squark gets a universal (mass) ${ }^{2} m_{0}^{2}$ (which may be very large but cancels in the difference) and a mass from gauge interactions (which govern the $m_{1 / 2}^{2}$ and $D$ term contributions) that are generation blind. The universal nature of $m_{0}^{2}$ arises from conditions (i) and (iv) of Sec. 9 which guarentees that the super Higgs fields communicate with the physical fields in a generation independent fashion.

(iii) Squarks ( $i=3$ ) and Radiative Breaking Masses

The $\tilde{b}_{R}$ squark behaves as the first two generation $d$-squarks with mass given by Eq. (112). However, as can be seen from Eqs. (105) and (106), the $\tilde{b}_{L}, \bar{t}_{L}$ and $\tilde{t}_{R}$ are strongly effected by the large $t$-quark Yukawa coupling. One finds for $\tilde{b}_{L}$ the result 


$$
\begin{aligned}
m_{\bar{b} L}^{2}=\frac{1}{2} m_{0}^{2}+m_{b}^{2} & +\frac{1}{2} m_{\tilde{U}}^{2}+\tilde{\alpha}_{G}\left[(4 / 3) f_{3}+(1 / 15) f_{1}\right] m_{1 / 2}^{2} \\
& +\left(-\frac{1}{2}+\frac{1}{3} \sin ^{2} \theta_{W}\right) M_{Z}^{2} \cos 2 \beta
\end{aligned}
$$

Further, the $t$-quark Yukawa coupling contribution to the $A$ term of Eq. (71) and the $\mu H_{1} H_{2}$ contribution to Eq. (71) cause mixing between the $\tilde{t}_{L}$ and $\tilde{t}_{R}$ states in the t-squark mass matrix:

$$
\left(\begin{array}{cc}
m_{\tilde{i}_{L}}^{2} & m_{t}\left(A_{t}+\mu \operatorname{ctn} \beta\right) \\
m_{t}\left(A_{t}+\mu \operatorname{ctn} \beta\right) & m_{\tilde{t}_{R}}^{2}
\end{array}\right) .
$$

where $m_{t}(Q)=\lambda_{t}(Q) v_{2}$ and $A_{t}$ is the $t$-quark $A$ parameter at the electroweak scale. The solution of the RGE yields

$$
\begin{gathered}
m_{i_{L}}^{2}=m_{Q}^{2}+m_{t}^{2}+\left[(-1 / 2)+(2 / 3) \sin ^{2} \theta_{W}\right] M_{Z}^{2} \cos ^{2} \beta \\
m_{\bar{t}_{R}}^{2}=m_{U}^{2}+m_{t}^{2}+(-2 / 3) \sin ^{2} \theta_{W} M_{Z}^{2} \cos 2 \beta
\end{gathered}
$$

where

$$
\begin{aligned}
m_{U}^{2}=\frac{1}{3} m_{0}^{2} & +\frac{2}{3} f A_{0} m_{1 / 2}-\frac{2}{3} k A_{0}^{2}+\frac{2}{3} h m_{0}^{2} \\
& +\left[\frac{2}{3} e+\tilde{\alpha}_{G}\left(\frac{8}{3} f_{3}-f_{2}+\frac{1}{3} f_{1}\right)\right] m_{1 / 2}^{2} \\
m_{Q}^{2}=\frac{2}{3} m_{0}^{2} & +\frac{1}{3} f A_{0} m_{1 / 2}-\frac{1}{3} k A_{0}^{2}+\frac{1}{3} h m_{0}^{2} \\
& +\left[\frac{1}{3} e+\tilde{\alpha}_{G}\left(\frac{8}{3} f_{3}+f_{2}-\frac{1}{15} f_{1}\right)\right] m_{1 / 2}^{2}
\end{aligned}
$$

where the functions $e(t), f(t), h(t), k(t)$ depend on $t=\ln \left[M_{G}^{2} / Q^{2}\right]$ and the $t$-quark Yukawa coupling, but are independent of $m_{0}, m_{1 / 2}, A_{0}, B_{0}$ and $\mu_{0}$. They are defined in Ibañez et al [33]. We also note that the three masses of Eq. (85) that enter into the radiative breaking equations (89) are obtained from Eqs. (94), (95), (98) and (99). One finds

$$
\begin{gathered}
m_{1}^{2}(t)=m_{0}^{2}+\mu^{2}(t)+g m_{1 / 2}^{2} \\
m_{2}^{2}(t)=\mu^{2}(t)+e(t) m_{1 / 2}^{2}+A_{0} m_{1 / 2} f+m_{0}^{2} h-A_{0}^{2} k \\
m_{3}^{2}=-B_{0} \mu^{2}(t)+r \mu_{0} m_{1 / 2}+8 A_{0} \mu_{0}
\end{gathered}
$$

where $\mu^{2}(t)=\mu_{0}^{2} q$ and $g(t), q(t), r(t), s(t)$ are defined in Ibañez et al [33].

\section{(iv) Charginos and Neutralinos}

As discussed in Sec. 4, supersymmetric dynamics causes $S U(2) \times U(1)$ gauginoHiggsino mixing after $S U(2) \times U(1)$ breaking. The mass diagonal states are two 
charged Winos (charginos) and 4 neutral Majorana Zinos (neutralinos). The chargino masses are [23]

$$
m_{\tilde{W}_{1,2}}=\frac{1}{2} \|\left[4 \nu_{+}^{2}+\left(\mu-\tilde{m}_{2}\right)^{2}\right]^{\frac{1}{2}} \mp\left[4 \nu_{-}^{2}+\left(\mu+\tilde{m}_{2}\right)^{2}\right]^{\frac{1}{2}} \mid
$$

where $\sqrt{2} \nu_{ \pm}=M_{W}(\sin \beta \pm \cos \beta)$. The neutralino masses are the four roots of the secular equation $f(\lambda)=0$ [23]

$$
\begin{aligned}
f(\lambda) & \equiv \lambda^{4}-\left(\tilde{m}_{1}+\tilde{m}_{2}\right) \lambda^{3}+\left(M_{Z}^{2}+\mu^{2}-\tilde{m}_{1} \tilde{m}_{2}\right) \lambda^{2} \\
& +\left[\left(\tilde{m}_{\gamma}-\mu \sin 2 \beta\right) M_{Z}^{2}+\left(\tilde{m}_{1}+\tilde{m}_{2}\right) \mu^{2}\right] \lambda \\
& +\left[\mu \tilde{m}_{\gamma} M_{Z}^{2} \sin 2 \beta-\tilde{m}_{1} \tilde{m}_{2} \mu^{2}\right]=0
\end{aligned}
$$

where $\tilde{m}_{\gamma}=\tilde{m}_{1} \cos ^{2} \theta_{W}+\tilde{m}_{2} \sin ^{2} \theta_{W}$. We label the Zino states by $\tilde{Z}_{i}, i=1 \ldots 4$, where $m_{\tilde{z}_{i}}<m_{\tilde{z}_{j}}$ for $i<j$.

\section{(v) Higgs Bosons}

Since there are two Higgs doublets, one is left with three neutral Higgs bosons [ $h$ and $H$ (CP-even states) and $A$ (CP-odd state)] and one charged boson $H^{ \pm}$. The tree level masses can be obtained from the mass matrix calculated from the Higgs potential of Eq. (83). One finds

$$
\begin{gathered}
m_{A}^{2}=m_{1}^{2}+m_{2}^{2}=2 m_{3}^{2} / \sin 2 \beta \\
m_{H^{ \pm}}^{2}=m_{A}^{2}+M_{W}^{2}
\end{gathered}
$$

where the last equality of Eq. (128) follows from Eq. (89). For the CP-even neutral Higgs, loop corrections to the light Higgs ( $h$ boson) are important since $h$ may be very light and a heavy top quark enhances the loop corrections. Keeping only the top sector in the loops, $m_{h}^{2}$ and $m_{H}^{2}$ become [39]:

$$
m_{h, H}^{2}=\frac{1}{2}\left[M_{Z}^{2}+m_{A}^{2}+\varepsilon \mp\left\{\left(M_{Z}^{2}+m_{A}^{2}+\varepsilon\right)^{2}-4 m_{A}^{2} M_{Z}^{2} \cos 2 \beta+\varepsilon_{1}\right\}^{1 / 2}\right]
$$

where $\varepsilon$ and $\varepsilon_{1}$ are the loop corrections. They can be written in terms of two $2 \times 2$ matrices $\nu_{i j}$ and $\Delta_{i j}$ according to

$$
\varepsilon=\operatorname{Tr} \Delta ; \varepsilon_{1}=-4(\operatorname{Tr} \nu \Delta+\operatorname{det} \Delta)
$$

where

$$
\begin{aligned}
\nu_{11} & =s^{2} M_{Z}^{2}+c^{2} m_{A}^{2} ; \nu_{22}=c^{2} M_{Z}^{2}+s^{2} m_{A}^{2} ; \nu_{12}=\nu_{21}=s c\left(M_{Z}^{2}+m_{A}^{2}\right) \\
\Delta_{11} & =x \mu^{2} y^{2} z ; \Delta_{12}=x \mu y\left(w+A_{t} y z\right)=\Delta_{21} \\
\Delta_{22} & =x\left(v+2 A_{t} y w+A_{i}^{2} y^{2} z\right)
\end{aligned}
$$

and 


$$
\begin{aligned}
x & =\frac{3 \alpha_{2}}{4 \pi} \frac{m_{t}^{4}}{M_{W}^{2} s^{2}} ; y=\frac{A_{t}+\mu c t n \beta}{m_{\tilde{t}_{1}}^{2}-m_{t_{2}}^{2}} ; z=2-w \frac{m_{\tilde{t}_{1}}^{2}+m_{\tilde{t}_{2}}^{2}}{m_{\tilde{t}_{1}}^{2}-m_{\tilde{t}_{2}}^{2}} \\
w & =\ln \left(m_{\tilde{t}_{1}}^{2} / m_{\tilde{t}_{2}}^{2}\right) ; v=\ln \left(m_{\tilde{t}_{1}}^{2} m_{\tilde{t}_{2}}^{2} / m_{t}^{4}\right)
\end{aligned}
$$

and $(s, c)=(\sin \beta, \cos \beta)$. Large correction can arise for the light Higgs $h$ boson for large $m_{t}$ due to the $m_{t}^{4}$ factor in the $x$ parameter. Loop corrections also exist for the $A$ and $H^{ \pm}$boson but these are small effects unless $A$ and $H^{ \pm}$are very light.

\section{MSSM as a Low Energy Approximation}

In the previous section, expressions were given allowing one to calculate each of the SUSY masses in terms of the Gut scale parameters. In addition, the mass parameters $m_{1}^{2}, m_{2}^{2}, m_{3}^{2}$ entering in the radiative breaking equations (91) were similarly expressed allowing one to use these relations to eliminate $B_{0}$ and $\mu_{0}$. (Since the masses are also expressed in terms of the VEVs $v_{1}$ and $v_{2}$, the loop corrections of Eq. (84) can also be calculated.) Thus the 32 SUSY masses can be expressed in terms of four parameters $m_{0}, m_{1 / 2}$ (or alternately the gluino mass, $m_{\tilde{g}}=\left(\alpha_{3} / \alpha_{G}\right) m_{1 / 2} \simeq$ $3.0 m_{1 / 2}$ ), $A_{0}$ (or alternately $A_{t}$, the $t$-quark $A$ parameter at the electroweak scale) and $\tan \beta$.

It is interesting to compare these results of supergravity grand unification with the MSSM. As discussed in Sec. 4, the MSSM assumes Eq. (108) for the gaugino masses as well as degeneracy of the first two generations of squarks [which was seen to be a good approximation to Eqs. (109)-(112)]. However, there are some places where the MSSM is not a good approximation to supergravity models:

(i) The third generation of squarks are badly split and except for $\tilde{b}_{R}$ not degenerate with the first two generations. (The usual MSSM analysis of data assume all six squarks (or sometimes five squarks) are degenerate.)

(ii) The sleptons will not be degenerate if $m_{0}$ is small (which is the case for the No-scale model). In addition, the MSSM depends on more parameters (and hence is less predictive) than the supergravity models. For example,

(iii) The chargino and neutralino masses depend on $\mu$, which is arbitrary in the MSSM but is determined (by radiative breaking) in supergravity models.

(iv) The $A$ Higgs boson mass, which by Eqs. (129) and (130) determine the other three Higgs boson masses, is arbitrary in the MSSM but depends on the same four parameters in supergravity models [see Eqs. (128) and (125)] and hence is correlated with other masses.

These differences are illustrated in Fig. 9. One sees that the third generation of squarks are badly split with the lightest stop, $\tilde{t}_{1}$, being the lightest squark. Also there are three light Winos and Zinos and three heavy ones, and one very light 


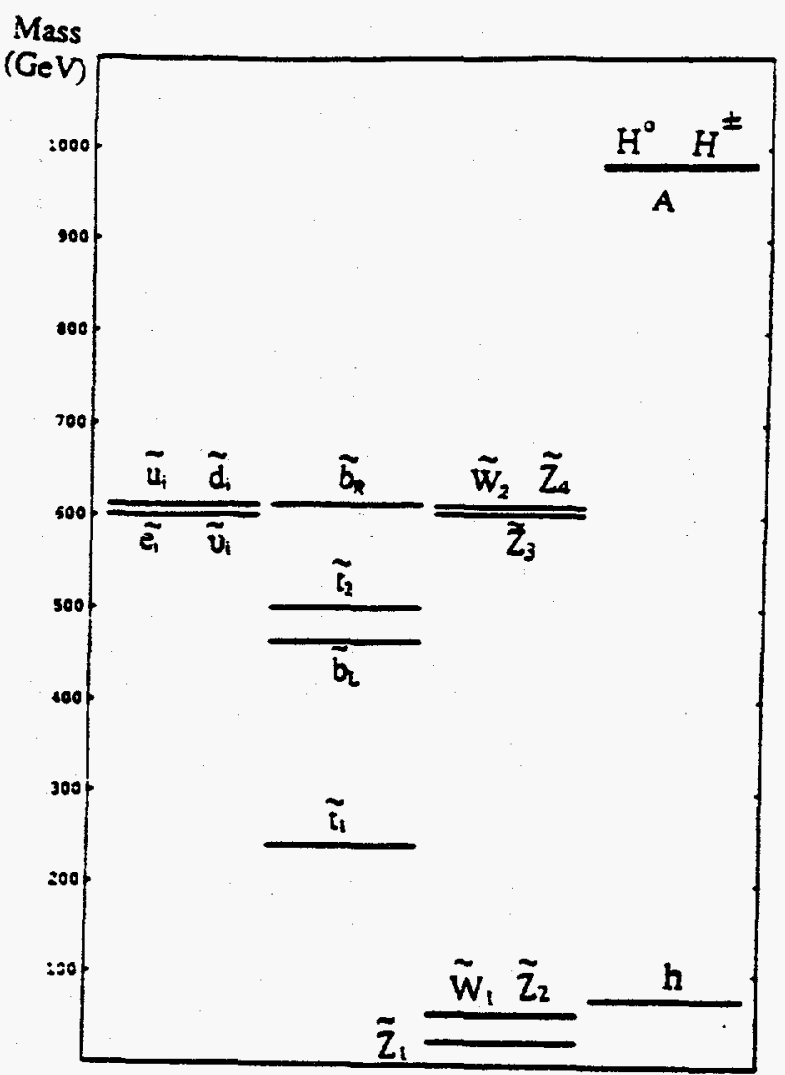

Figure 9: Predictions of 32 SUSY masses for supergravity grand unification for the case $m_{t}=$ $150 \mathrm{GeV}, m_{0}=600 \mathrm{GeV}, m_{\tilde{g}}=160 \mathrm{GeV}, \tan \beta=1.73, A_{t}=0.0(\mu<0)$. These parameters uniquely specify all masses. The first column is the sleptons and first two generations of squarks, the second column is the third generation of squarks, the third column is the charginos and neutralinos, and the last column the Higgs bosons.

Higgs $(h)$ and three heavy ones $\left(H, A, H^{ \pm}\right)$. The sleptons are nearly degenerate with the first two generations of squarks since $m_{0}$ is fairly large for this case.

\section{Threshold Corrections to Grand Unification}

In the discussion of the unification of the coupling constants in Sec. 6, it was assumed that all SUSY particles had a common mass $M_{S}$. As we have seen, the SUSY spectrum is split ranging from below $M_{Z}$ up to about $1 \mathrm{TeV}$. As a consequence there are threshold corrections to the RGE that describe the unification of the couplings. Similarly at the Gut scale, as seen in the $S U(5)$ example of Sec. 10 , we may expect an array of superheavy non-degenerate states with masses of $O\left(M_{G}\right)$ which will also have threshold corrections. These corrections can modify the grand unification results and some analyses of these problems have been given [40]-[43].

We consider first the case of the low energy thresholds at $M_{s}$. For a fixed Gut 
model defined by specific Gut parameters $m_{0}, m_{1 / 2}$ etc., we saw we can calculate all the SUSY masses and thus be able to insert the thresholds in the RGE running of the coupling constants. Each threshold causes a kink in $\alpha_{i}^{-1}$ (Fig. 10) which shifts the positions of $M_{G}$ and $\alpha_{G}$. However, the positions of each SUSY mass depends on $M_{G}$ and $\alpha_{G}$ (from the running of the RGE) as can be seen in Sec. 12. Hence one has an additional dependence of the final grand unification point on the position of the threshold. Thus a complicated numerical analysis is needed to deal with this.

It is possible to parameterize the SUSY thresholds phenomenologically [42]. Thus integrating Eq. (32) from $\mu=M_{G}$ to $\mu=M_{Z}$ gives:

$$
\alpha_{i}^{-1}\left(M_{Z}\right)=\alpha_{G}^{-1}+b_{i} t_{0}-\Delta_{i}+2-\text { loop }
$$

where $t_{0}=\ln \left[M_{G} / M_{z}\right] / 2 \pi$ and $\Delta_{i}$ are the SUSY threshold pieces,

$$
\Delta_{i}=\frac{1}{2 \pi} \sum b_{i}^{(\alpha)} \ln \left(M_{a} / M_{z}\right)
$$

Here $b_{i}^{(a)}$ is the contribution of the ath particle to the beta functions $\beta_{i}=b_{i} \alpha_{i}^{2} / 2 \pi$ (each particle decoupling at its mass as one proceeds downwards from $M_{G}$ ). The $\Delta_{i}$ are of course functions of the Gut parameters and can be explicitly calculated using Sec. 12. Instead, one may use a phenomenological parameterization defined by three masses $M_{i}, i=1,2,3$ :

$$
\sum b_{i}^{(a)} \ln \left(M_{a} / M_{Z}\right) \equiv\left(b_{i}^{M S S M}-b_{i}^{S M}\right) \ln \left(M_{i} / M_{Z}\right)
$$

Clearly, if all SUSY particles are degenerate, $M_{i}=M_{S}$, but otherwise the $M_{i}$ are distinct. Now one may use the place where $\alpha_{1}$ and $\alpha_{2}$ intersect to define the grand unification point $\mu=M_{G}$ and the value of $\alpha_{G}$. Then by requiring also $\alpha_{3}\left(M_{G}\right)=\alpha_{G}$ one can predict the value of $\alpha_{3}\left(M_{Z}\right)$ (which can then be compared with experiment). The threshold contributions to $\alpha_{3}\left(M_{z}\right)$ are

$$
25 \ln \left(M_{1} / M_{Z}\right)-100 \ln \left(M_{2} / M_{Z}\right)+56 \ln \left(M_{3} / M_{Z}\right) \equiv-19 \ln \left(M_{S}^{e f f} / M_{Z}\right)
$$

which defines an effective $M_{S}$. Note however, that $M_{S}^{e f f}$ is different from the average SUSY mass since it is an average of logarithms weighted by $\beta$ function factors, one of which is negative. Thus it is even possible to have $M_{s}^{\text {eff }}<M_{z}$. In general one finds that these threshold corrections are about as large as the current error flags, and hence will become more important as experimental errors decrease.

An important point to remember in considering the above analysis is that the energy scale of supersymmetry breaking is the same of $S U(2) \times U(1)$ breaking. This can be seen, for example, in Fig. 9, where the mass spectrum of the third generation squarks, the Winos and Zinos etc. are not $S U(2) \times U(1)$ invariant. (In fact, the $\tilde{Z}_{1,2}$ and $\tilde{W}_{1}$ may lie below $M_{Z}$.) Thus in proceeding up in energy from $M_{Z}$, one cannot assume $S U(2) \times U(1)$ invariance holds in the SUSY particle region, as is sometimes done. 


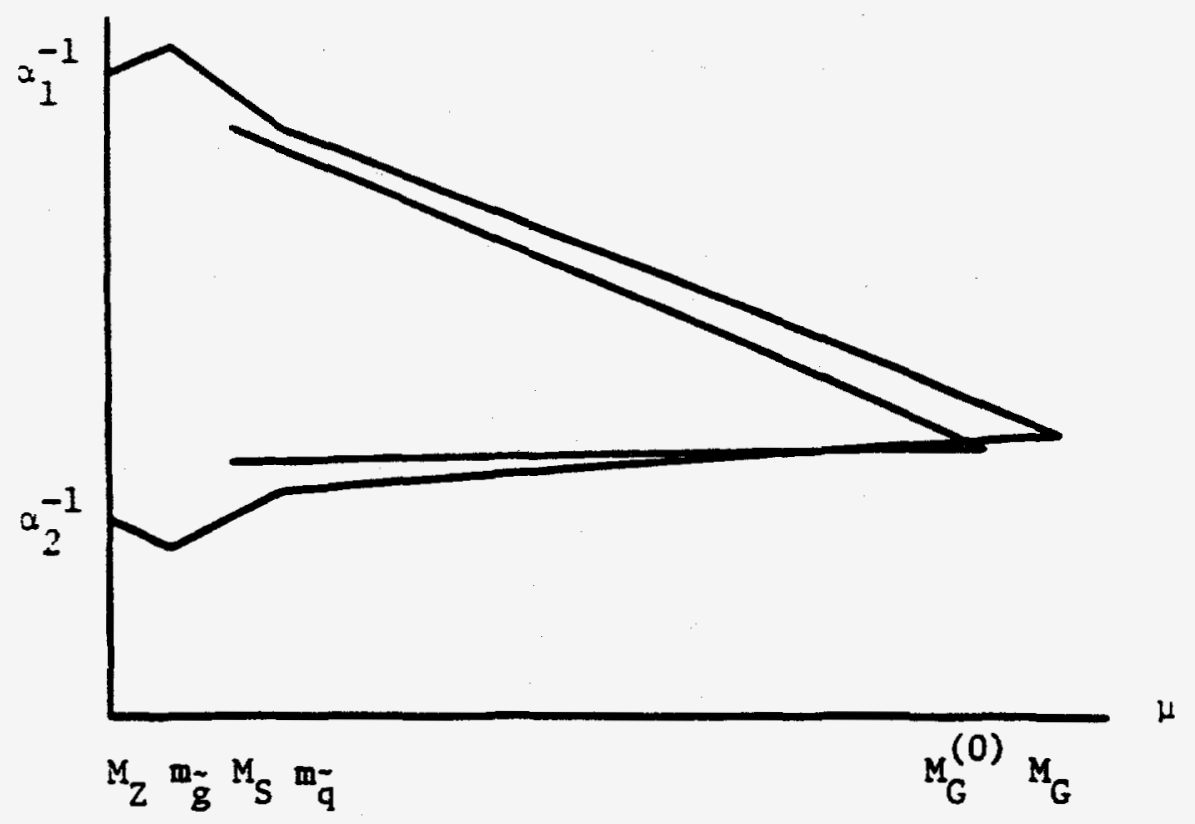

Figure 10: Schematic diagram showing effects of SUSY mass thresholds. The values of $M_{G}$ and $\alpha_{G}$ are shifted when threshold effects are taken into account.

We turn next to consider the Gut scale thresholds. The physics at the Gut scale is unknown, and so treatment of threshold effects here are more speculative. In order to learn the general nature of these effects we consider the $S U(5)$ model of Eqs. (77) and (78). From explicit calculation of the mass matrix one finds that the superheavy particles arising from the breaking of $S U(5)$ are the following:

(i) Two color Higgs triplet chiral multiplets transforming under $S U(3)_{C} \times S U(2)_{L}$ as $(3,1)$ and $(\overline{3}, 1)$ with mass $M_{H_{3}}=5 \lambda_{2} M$.

(ii) Two massive vector multiplets (consisting each of a massive vector boson, Dirac spinor and hermitian scalar) transforming as $(3,2)$ and $(\overline{3}, 2)$ with mass $M_{V}=5 \sqrt{2} g M$, where $\alpha_{G}=g^{2} / 4 \pi$.

(iii) Those components of the chiral multiplets of $\Sigma_{Y}^{X}$ not absorbed by the vector bosons in their mass growth. These are two degenerate representations transforming as $(8,1)$ and $(1,3)$ with masses $M_{\Sigma}^{B}=5 \lambda_{1} M / 2=M_{\Sigma}^{3}$ and a SM singlet with mass $M_{2}^{e}=\lambda_{1} M / 2$.

Upper bounds on the $\lambda_{1,2}$ arise by requiring that the model stay within the perturbative domain. We take this to mean $\lambda_{1,2} \leq 2$, or $\alpha_{\lambda_{1,2}}=\lambda_{1,2}^{2} / 4 \pi \leqslant 1 / 3$. Then since $\alpha_{G}^{-1} \simeq 25$ one has

$$
\frac{M_{H_{3}}}{M_{V}}=\frac{\lambda_{2}}{\sqrt{2} g} \lesssim 2 ; \quad \frac{M_{5}^{8,3}}{M_{V}} \lesssim 1 ; \quad \frac{M_{2}^{0}}{M_{V}} \lesssim 0.2
$$


Thus the superheavy particles cannot get much larger than $M_{V}$. Since the superheavy particles are expected to be $O\left(M_{G}\right)$ we will also require $\lambda_{1,2}>0.01$ or $\alpha_{\lambda_{1,2}} \gtrsim 10^{-5}$.

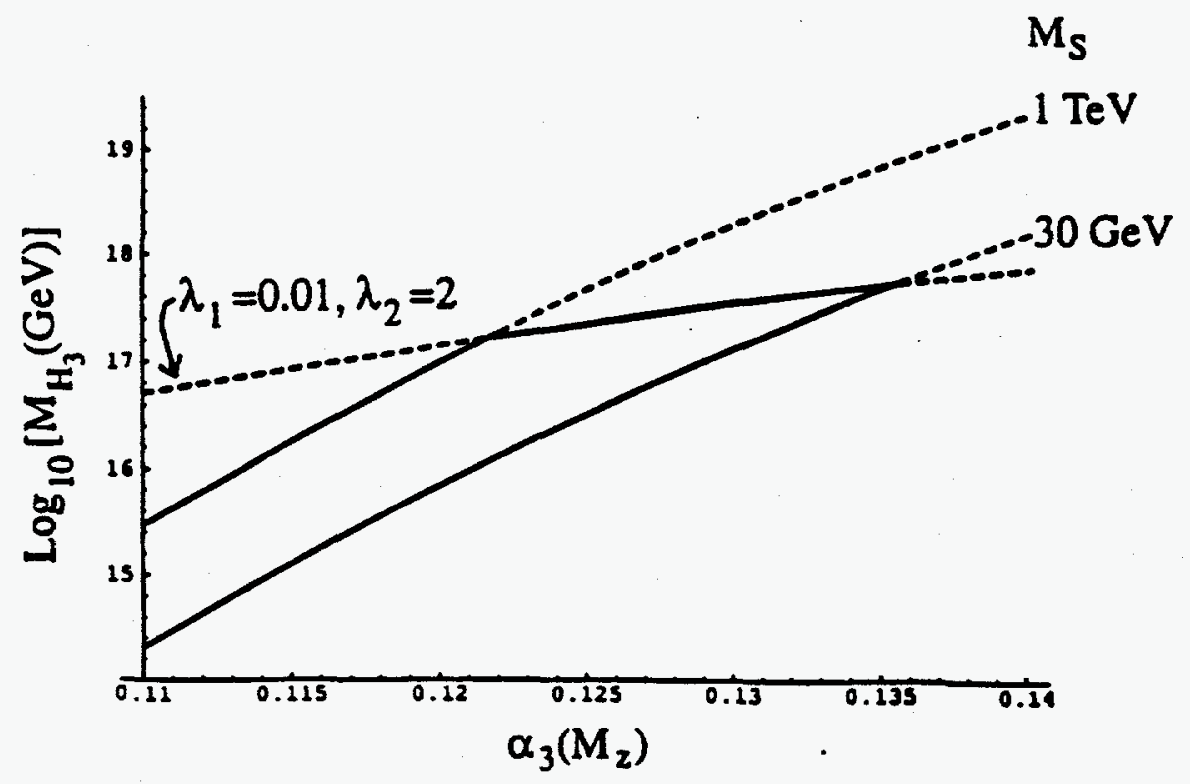

Figure 11: Grand unification including Gut thresholds of the model of Eq. (77). The allowed region is that bounded by the solid quadrilateral.

One may now run the RGE up to the Gut scale, taking into account the additional Gut thresholds. An accurate analysis requires inclusion of two loop effects. Fig. 11 [43] shows the effects of the Gut scale thresholds neglecting the low energy SUSY thresholds. Grand unification implies a correlation between $M_{H_{3}}$ and $\alpha_{3}\left(M_{Z}\right)$. For $\lambda_{1}>0.01$ or $\lambda_{2}<2$, the horizontal line moves downwards. The effective $M_{S}$ is assumed to obey the bounds $30 \mathrm{GeV}<M_{S}<1 \mathrm{TeV}$. The solid quadrilateral thus represents the allowed region of grand unification. The model thus predicts $\alpha_{3}\left(M_{z}\right)<0.135$ consistent with the experimental value of $\alpha_{3}\left(M_{z}\right)=0.118 \pm 0.007$. At the $1 \sigma$ upper bound of $\alpha_{3}\left(M_{Z}\right)=0.125$, one finds $M_{H_{3}}<2 \times 10^{17} \mathrm{GeV}$. Clearly, an accurate determination of $\alpha_{3}\left(M_{Z}\right)$ would allow further tests of this model as $M_{H_{3}}$ is related to the proton decay rate.

\section{Models with Proton Decay}

The analysis to obtain predictions of supergravity Gut models can proceed as follows: One calculates the 32 SUSY masses in terms of the $4+1$ parameters $m_{0}, m_{1 / 2}, A_{0}, \tan \beta$ and $m_{t}$. One then varies these parameters over their entire range subject to the conditions that (i) Radiative breaking of $S U(2)_{L} \times U(1)_{Y}$ occurs, and (ii) Experimental bounds on SUSY masses are not violated. One then gets the allowed mass bands and correlations between SUSY masses. These are the experimental predictions of the model. 
It is possible to reduce the size of the allowed parameter space by imposing additional constraints. Three possibilities that have been considered are: (i) Constraints due to experimental bounds on proton decay, (ii) Cosmological constraints from SUSY candidates for dark matter, and (iii) Constraints from conditions on Yukawa couplings at the Gut scale [e.g. $\left.\lambda_{b}\left(M_{G}\right)=\lambda_{\tau} M_{G}\right)$ ]. We consider the first of these here.

There are two main decay modes possible in SUSY Gut models: $p \rightarrow e^{+}+$ $\pi^{0}$ and $p \rightarrow \bar{\nu}+K^{+}$. The current 90\% CL bounds from Kamiokande and IMB are [44]

$$
\begin{aligned}
& \tau\left(p \rightarrow e^{+} \pi^{0}\right)>5.5 \times 10^{32} \mathrm{yr} \\
& \tau(p \rightarrow \bar{\nu} K)>1.0 \times 10^{32} \mathrm{yr}
\end{aligned}
$$

The decay $p \rightarrow e^{+} \pi^{0}$ can occur in both SUSY and non-SUSY models. It proceeds through the superheavy vector bosons ( $X^{\mu}, Y^{\mu}$ in $S U(5)$ theory) with mass $M_{V}$ as shown in Fig. 12. For SUSY models, the lifetime for $p \rightarrow e^{+} \pi^{0}$ is given by [42]:

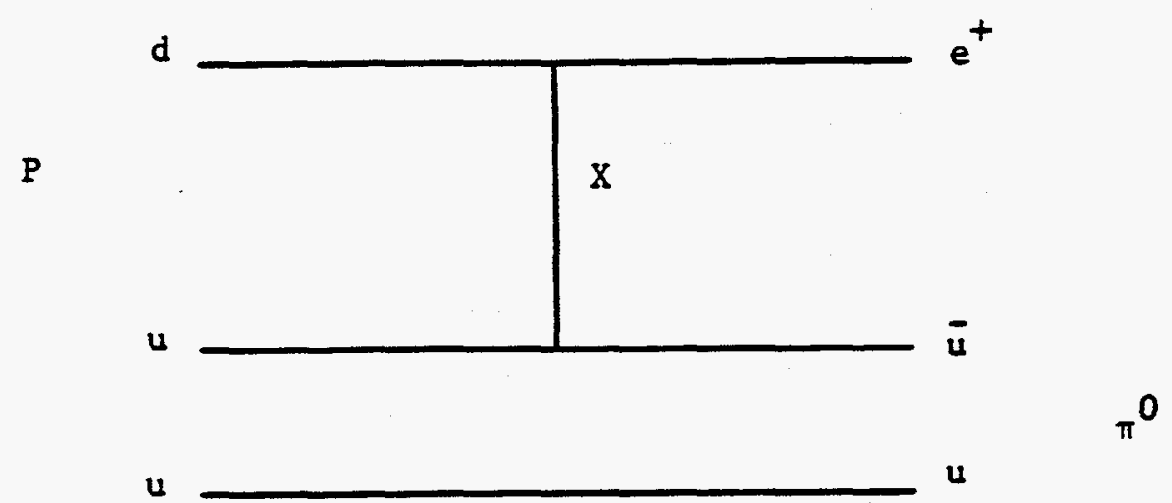

Figure 12: Proton decay diagram for the mode $p \rightarrow e^{+}+\pi^{0}$.

$$
\tau\left(p \rightarrow e^{+} \pi^{0}\right) \simeq\left(\frac{M_{V}}{3.5 \times 10^{14} \mathrm{GeV}}\right)^{4} 10^{31 \pm 1} y r
$$

Super Kamiokande plans to be sensitive to this mode up to about $1 \times 10^{34} \mathrm{yr}$. To be observable at this level requires (at about $90 \%$ CL) that $M_{V} \leqslant 5 \times 10^{15} \mathrm{GeV}$. Since the mean $M_{G}$ value is $M_{G} \simeq 1.5 \times 10^{16} \mathrm{GeV}$, it is doubtful that this model will be observable at Super Kamiokande.

The decay $p \rightarrow \bar{\nu}+K^{+}$is specifically a supersymmetric mode and hence observation of this decay would be a real indication of the validity of supergravity Guts. However, it does not necessarily arise in all supergravity models. We consider here "SU(5)-type" proton decay models defined as follows:

(i) The Gut group $G$ contains an $S U(5)$ subgroup [or is $S U(5)]$. 
(ii) The matter that remains light after $G$ breaks to $S U(3)_{C} \times S U(2)_{L} \times U(1)_{Y}$ at $M_{G}$ is embedded in the usual way in the 10 and $\overline{5}$ representations of the $S U(5)$ subgroup.

(iii) After $G$ breaks, there are only two light Higgs doublets which interact with matter, and these are embedded in the 5 and $\overline{5}$ of the $S U(5)$ subgroup.

(iv) There is no discrete symmetry or fine tuning condition that forbids the proton decay amplitude.

The above conditions can hold for a variety of groups besides $S U(5)$ e.g. $O(10), E_{6}$ etc. Remarkably, models obeying (i) - (iv) give rise to a universal decay amplitude $[45,46]$ for $p \rightarrow \bar{\nu}+K^{+}$arising from the exchange of the superheavy Higgsino color triplet of mass $M_{H_{3}}$. A characteristic diagram is shown in Fig. 13.

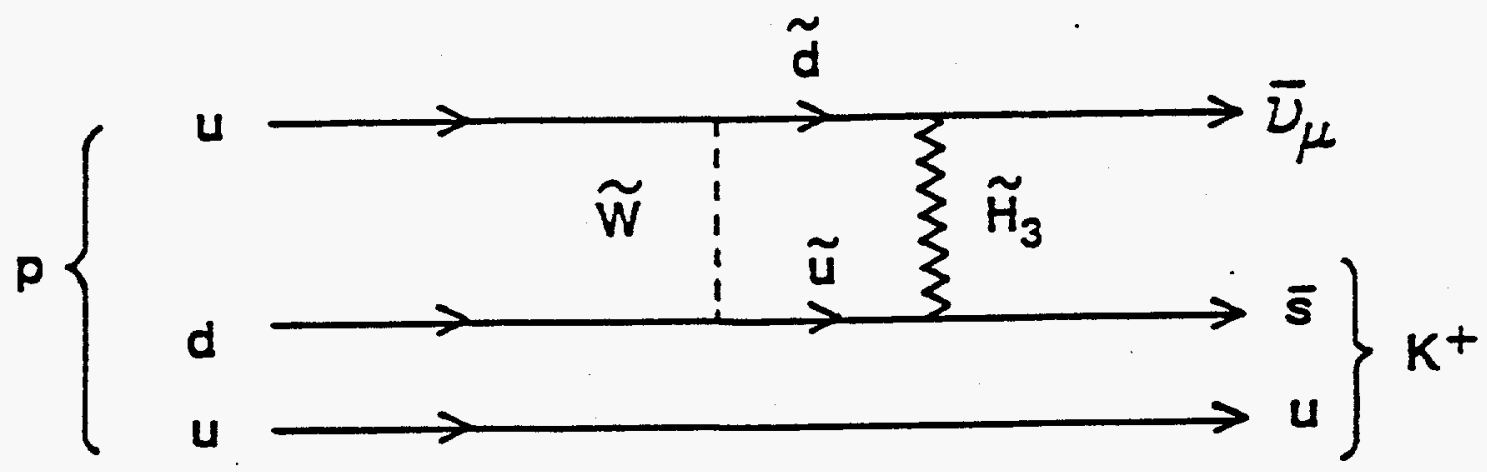

Figure 13: One diagram contributing to the decay amplitude for $p \rightarrow \bar{\nu}+K^{+}$. The Wino "dressing" converts quarks to squarks and the $\tilde{H}_{3}$ vertices violate baryon and lepton number. There are additional diagrams with $\bar{\nu}_{e}, \bar{\nu}_{\tau}$ final states. Also the CKM matrix elements appear at the $\tilde{W}$ vertices allowing all three generations to enter in the loop.

Proton decay is characteristic of grand unified models and one might ask under what circumstances it can be suppressed. It appears difficult to find a discrete symmetry that can suppress the $p \rightarrow \bar{\nu} K^{+}$decay without introducing additional Higgs doublets (which would ruin grand unification) or produce some other illness. The only other natural way of suppressing the decay is by violating condition (ii). This is done in the flipped $S U(5) \times U(1)$ model where the interchanges $u \leftrightarrow d$ and $e \leftrightarrow \nu$ in the particle embeddings suppresses proton decay. We will come back to this model later.

Conditions (i) - (iv) imply the existance of an $S U(5)$ invariant contribution to the Yukawa part of the superpotential of

$$
W_{Y}=\lambda_{i j}^{1} \varepsilon_{X Y Z W U} H_{1}^{X} M_{i}^{Y Z} M_{j}^{W U}+\lambda_{i j}^{2} \bar{H}_{2 X} \bar{M}_{i Y} M_{j}^{X Y}
$$

where we have used the notation of Sec. 10 . It is the superheavy $H_{1}^{a}$ and $\bar{H}_{2 a}$ couplings in Eq. (142) which give rise to the $p$-decay interaction. One may eliminate 
these fields to obtain an effective dimension five interaction scaled by $1 / M_{H_{\mathbf{3}}}$. The total decay rate is

$$
\Gamma\left(p \rightarrow \bar{\nu} K^{+}\right)=\sum_{i} \Gamma\left(p \rightarrow \bar{\nu}_{i} K^{+}\right)
$$

where $\bar{\nu}_{i}=\left(\bar{\nu}_{e}, \bar{\nu}_{\mu}, \bar{\nu}_{\tau}\right)$. The calculation of $\Gamma\left(p \rightarrow \bar{\nu} K^{+}\right)$is rather lengthy. We state here the final result [46] for reference. The first generation contributions are negligible, and so one may limit $i$ to $i=\mu, \tau$. One can write

$$
\Gamma\left(p \rightarrow \bar{\nu}_{i} K^{+}\right)=C\left(\frac{\beta_{p}}{M_{H_{s}}}\right)^{2}|A|^{2}\left|B_{i}\right|
$$

where $C$ is a chiral current algebra factor (Chadha and Daniels [45])

$$
C=\frac{m_{N}}{32 \pi f_{\pi}^{2}}\left[\left(1+\frac{m_{N}(D+F)}{m_{B}}\right)\left(1-\frac{m_{K}^{2}}{m_{N}^{2}}\right)\right]^{2}
$$

$\left(D=0.76, F=0.48, f_{\pi}=139 \mathrm{MeV}, m_{N}=938 \mathrm{MeV}, m_{B}=1154 \mathrm{MeV}, m_{K}=495 \mathrm{MeV}\right)$. The factor $A$ is

$$
A=\frac{\alpha_{2}^{2}}{2 M_{W}^{2}} m_{2} m_{c} V_{21}^{\dagger} V_{21} A_{L} A_{S}
$$

where $V_{i j}$ are CKM elements, $A_{L, S}$ are renormalization group factors to take the operator at the Gut scale to the proton scale (Ellis et al [45]). We find $A_{L}=0.283$, $A_{s}=0.833$. The quantity $\beta_{p}$ describes the quark content of the proton,

$$
\beta_{p} U_{L}^{\gamma}=\varepsilon_{a b e} \varepsilon_{\alpha \beta}<o\left|d_{a L}^{\alpha} u_{b L}^{\beta} u_{c L}^{\gamma}\right| p>
$$

where $U_{L}^{\gamma}$ is the proton wave function, $d_{a L}^{\alpha}, u_{b L}^{\beta}$ are quark operators $(\alpha, \beta=$ spinor indices). $\beta_{p}$ has been evaluated by lattice gauge theory [47]:

$$
\beta_{p}=(5.6 \pm 0.5) \times 10^{-3} \mathrm{GeV}^{3}
$$

The loop integral $B_{i}$ may be written as

$$
B_{i}=\frac{m_{i}^{d} V_{i 1}^{\dagger}}{m_{e} V_{21}^{\dagger}}\left[P_{2} B_{2 i}+\frac{m_{i} V_{31} V_{32}}{m_{e} V_{21} V_{22}} P_{3} B_{3 i}\right] \frac{1}{\sin 2 \beta}
$$

where $m_{i}^{d}$ are $d$-quark masses and $B_{j i}$, the contribution of $j$ th generation particles in the loop to the amplitude for $p \rightarrow \bar{\nu}_{i} K^{+}$may be written as

$$
B_{j i}=F\left(\tilde{u}_{i}, \tilde{d}_{j}, \tilde{W}\right)+\left(\tilde{d}_{j}+\tilde{e}_{j}\right)
$$

where

$$
\begin{aligned}
& F\left(\tilde{u}_{i}, \tilde{d}_{j}, \tilde{W}\right)=\left[E \cos \gamma_{-} \sin \gamma_{+} \tilde{f}\left(\tilde{u}_{i}, \tilde{d}_{j}, \tilde{W}_{1}\right)+\right. \\
& \left.\cos \gamma_{+} \sin \gamma-\tilde{f}\left(\tilde{u}_{i}, \tilde{d}_{j}, \tilde{W}_{2}\right)\right]-\frac{1}{2} \frac{\delta_{i 3} m_{i}^{u} \sin 2 \delta_{u i}}{\sqrt{2} M_{W} \sin \beta}\left[\left\{E \sin \gamma-\sin \gamma_{+} f\left(\tilde{u}_{i 1}, \tilde{d}_{j}, \tilde{W}_{1}\right)\right.\right. \\
& \left.\left.\quad-\cos \gamma_{-} \cos \gamma_{+} f\left(\tilde{u}_{i 1}, \tilde{d}_{j}, \tilde{W}_{2}\right)\right\}-\left\{\tilde{u}_{i 1} \rightarrow \tilde{u}_{i 2}\right\}\right]
\end{aligned}
$$


with the definitions $\gamma_{ \pm}=\beta_{+} \pm \beta_{-}$,

$$
\begin{gathered}
\sin 2 \beta_{ \pm}=\frac{\left(\mu \mp \tilde{m}_{2}\right)}{\left[4 \nu_{ \pm}^{2}+\left(\mu \pm \tilde{m}_{2}\right)^{2}\right]^{1 / 2}} ; \sqrt{2} \nu_{ \pm}=M_{W}(\sin \beta \pm \cos \beta) \\
\sin 2 \delta_{u 3}=\frac{-2\left(A_{t}+\mu \operatorname{ctn} \beta\right) m_{t}}{m_{\tilde{t}_{i}}^{2}-m_{\tilde{2}_{2}}^{2}} \\
E= \begin{cases}+1, & \sin 2 \beta>\mu \tilde{m}_{2} / M_{W}^{2} \\
-1, & \sin 2 \beta<\mu \tilde{m}_{2} / M_{W}^{2}\end{cases}
\end{gathered}
$$

and

$$
\begin{aligned}
\tilde{f}\left(\tilde{u}_{i}, \tilde{d}_{j}, \tilde{W}_{k}\right) & =\sin ^{2} \delta_{u i} f\left(\tilde{u}_{i 1}, \tilde{d}_{j}, \tilde{W}_{k}\right) \\
& +\cos ^{2} \delta_{u i} f\left(\tilde{u}_{i 2}, \tilde{d}_{j}, \tilde{W}_{k}\right)
\end{aligned}
$$

with $\tilde{u}_{i 1}$ and $\tilde{u}_{i 2}$ being the $u$-squark mass states and

$$
f(a, b, c)=\frac{m_{c}}{m_{b}^{2}-m_{c}^{2}}\left[\frac{m_{b}^{2}}{m_{a}^{2}-m_{b}^{2}} \ln \left(\frac{m_{a}^{2}}{m_{b}^{2}}\right)-\left(m_{b} \rightarrow m_{c}\right)\right]
$$

Again $j=1$ gives a neglegible contribution and we have include L-R mixing only for the $t$-squarks.

In Eq. (148), the phases $P_{j}=e^{i \alpha_{j}}, j=2,3$ appear. These are new CP violating phases (independent of the CKM phase) that enter in the dimension five operators. Thus there are two limiting cases: $P_{3} / P_{2}=-1$, destructive interference between the second and third generation contributions to the loop, and $P_{3} / P_{2}=+1$, constructive interference.

It is convenient to define the quantity

$$
B \equiv\left[\left|B_{2}\right|^{2}+\left|B_{3}\right|^{2}\right]^{\frac{1}{2}}\left[M_{S} / 10^{2.4} \mathrm{GeV}\right]^{0.33} \times 10^{6} \mathrm{GeV}^{-1}
$$

and using Eq. (40) (to account for the anti-correlation between $M_{S}$ and $M_{G}$ ), the Kamiokande experiment bound, Eq. (140), can be written as

$$
B \lesssim 100\left(\frac{M_{H_{2}}}{M_{G}}\right) \mathrm{GeV}^{-1}
$$

for $\beta_{p}=5.6 \times 10^{-3} \mathrm{GeV}^{3}$. The quantity $B$ is a function of the SUSY masses (squarks, sleptons, Winos) and so the experimental bound on $B$ becomes a constraint on the SUSY mass spectrum.

\section{Predictions for Models with Proton Decay}

We now discuss what predictions can be made for the SUSY mass spectrum for models possessing $S U(5)$-type proton decay. We will see, in fact, that with sensitivity expected in future underground and accelerator experiments, it should be possible to test whether these models are right or wrong. 
Our procedure is to allow the basic parameters, $m_{0}, m_{1 / 2}, A_{0}, \tan \beta$ and $m_{t}$ to vary arbitrarily subject to the following constraints: (i) Radiative breaking of $S U(2)_{L} \times U(1)_{Y}$ occurs. (ii) Current experimental bounds on SUSY masses are obeyed. (iii) Two theoretical constraints are imposed: (a) No extreme fine tuning of parameters are allowed which we quantify by requiring $m_{\tilde{q}}, m_{\tilde{g}}<1 \mathrm{TeV}$, and (b) $M_{H_{\mathfrak{g}}}$ is $O\left(M_{G}\right)$ which we quantify by requiring $M_{H_{3}} / M_{G}<10$.

Condition (iiia) accepts fine tuning up to about the $1 \%$ level which is about the limit of loop corrections. Condition (iiib) implies $M_{H_{3}} \lesssim 2 \times 10^{17} \mathrm{GeV}$. Any larger value of $M_{H_{3}}$ would need to include Planck physics corrections and the supergravity Gut theory would not be self-contained. Note also that in the simple Gut model discussed in Sec. 13, Fig. 9, the experimental value of $\alpha_{3}\left(M_{Z}\right)$ requires a bound of this size for grand unification to occur.

We review briefly now the procedure of calculation: (i) Run the one loop SUSY RGE from $M_{G}$ to $M_{Z}$ and impose radiative breaking on the Higgs potential. (ii) Calculate all 32 SUSY masses as a function of $m_{0}, m_{1 / 2}, A_{0}, \tan \beta$ and $m_{t}$. (iii) Limit the parameter space so that the experimental bounds on SUSY masses are obeyed. (iv) Limit the parameter space so that proton decay bounds are obeyed (i.e. $B<1000 \mathrm{GeV}^{-1}$ ). (v) Allow parameters to vary over the remaining allowed regions and obtain the allowed bands of SUSY masses and the correlations between masses. These are the predictions that can be checked experimentally.

The calculations discussed below are made under the following conditions: (1) In (i) above we neglect loop corrections to the Higgs potential. As discussed above, these are generally small [37]. (2) However, in (ii) above we do include the loop corrections to the light Higgs mass $m_{h}$ as these may be large, particularly for large $m_{t}$. (3) In calculating $B$, we assume the distructive interference possibility i.e. $P_{2} / P_{3}=-1$. This minimizes as much as possible the effects of proton decay on the SUSY mass spectrum. (4) Central values of CKM matrix elements are used: $V_{31}=0.011, V_{32}=-0.042, V_{13}=-0.002$. (5) Only the $t$-quark Yukawa coupling was included. This is a good approximation for $\tan \beta<10$, which we will see is generally valid.

The proton decay amplitude function $B$ of Eq. (156) is a complicated function of SUSY masses. We can get a qualitative picture of what the proton decay constraint implies by considering only the second generation contribution in the limit of large $m_{0}$ (i.e. $m_{0}^{2} \gg M_{Z}^{2}, \tilde{W}_{2}^{2}$ ). Then $B$ takes the simple form

$$
B_{2} \simeq-\frac{2 \alpha_{2}}{\alpha_{3} \sin 2 \beta} \frac{m_{\bar{g}}}{m_{\bar{q}}^{2}} \times 10^{\circ}
$$

where the RGE give $m_{\tilde{q}}^{2} \cong m_{0}^{2}+(0.65) m_{\tilde{g}}^{2}$. An upper bound on $\left|B_{2}\right|$ generally implies (1) an upper bound on $m_{\tilde{g}}$ (unless $m_{\tilde{g}}$ is very large, i.e. $m_{\tilde{g}}^{2}>m_{0}^{2}$ as then $\left|B_{2}\right| \sim 1 / m_{\tilde{g}}$ ), (2) a lower bound on $m_{0}$ so that $m_{\tilde{q}}^{2}$ does not get too small (again unless $m_{\tilde{g}}$ is very large), and (3) an upper bound on $\tan \beta$ so that $\sin 2 \beta$ does not get too small.

The above qualititive features are exhibited in Fig. 14 and Fig. 15 [48]. These figures also exhibit the fact that current proton decay data for $p \rightarrow \bar{\nu} K^{+}$requires $M_{H_{3}} \gtrsim 1 \times 10^{16} \mathrm{GeV}\left(M_{H_{3}} / M_{G} \gtrsim 2 / 3\right)$. In the Gut model of Sec. 10, then, Eq. (138) 


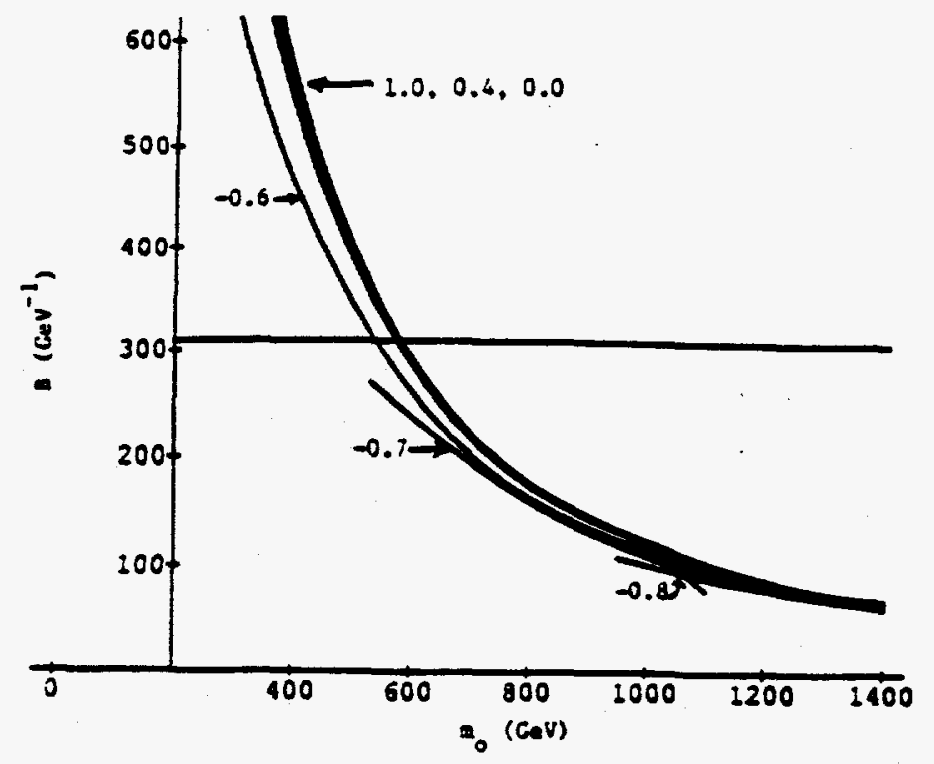

Figure 14: $B$ as a function of $m_{0}$ for $m_{t}=125 \mathrm{GeV}, m_{\tilde{g}}=160 \mathrm{GeV}, \tan \beta=1.73, \mu<0$. The low $m_{0}$ cutoff for $A_{t}<0$ is due to $\bar{t}_{1}$ turning tachyonic.

and the bound on $M_{V}$ of Eq. (141) would make it unlikely that Super Kamiokande would detect the $p \rightarrow e^{+} \pi^{0}$ mode (and if it did, the model predicts that the $p \rightarrow \bar{\nu} K^{+}$ mode would be very copious). The bound on $M_{H_{3}}$ also implies $\alpha_{3}\left(M_{Z}\right) \gtrsim 0.114$ in this model.

It is convenient to consider the results as a function of $M_{H_{3}}$ :

1. $M_{H_{3}} / M_{G}<3$

For this case, where $M_{H_{3}}$ is relatively small, one finds that $m_{0} \gtrsim 500 \mathrm{GeV}$ and $m_{\tilde{g}} \lesssim 450 \mathrm{GeV}$ (as illustrated in Figs. 12 and 13). In addition one finds $\left|A_{t}\right| / m_{0} \lesssim 1.5$ and $1.1 \lesssim \tan \beta \lesssim 5$. One expects therefore that the squarks (with the possible exception of $\tilde{t}_{1}$ ) and probably also gluinos will require the SSC or LHC to be detected. The $t$ quark and $h$ boson are limited to $m_{t}<180 \mathrm{GeV}$ and $m_{h}<110 \mathrm{GeV}$.

2. As one increases $M_{H_{3}}$, the lower bound on $m_{0}$ decreases and the upperbound on $m_{\tilde{j}}$ increases. Thus for $M_{H_{\mathfrak{j}}} / M_{G} \gtrsim 7, m_{\tilde{j}}$ can saturate its upper bound of $1 \mathrm{TeV}$ (though usually a large $m_{\bar{g}}$ requires a small $m_{0}$.) The allowed bands on $\tan \beta$ and $A_{t}$ widen a little (e.g. $\tan \beta \lesssim 8$ ).

3. Perhaps the most remarkable results is a set of scaling relations that hold among the charginos, neutralinos and gluino over most of the allowed parameter space $[48,49]$

$$
2 m_{\tilde{Z}_{1}} \cong m_{\tilde{W}_{1}} \cong m_{\tilde{Z}_{2}}
$$




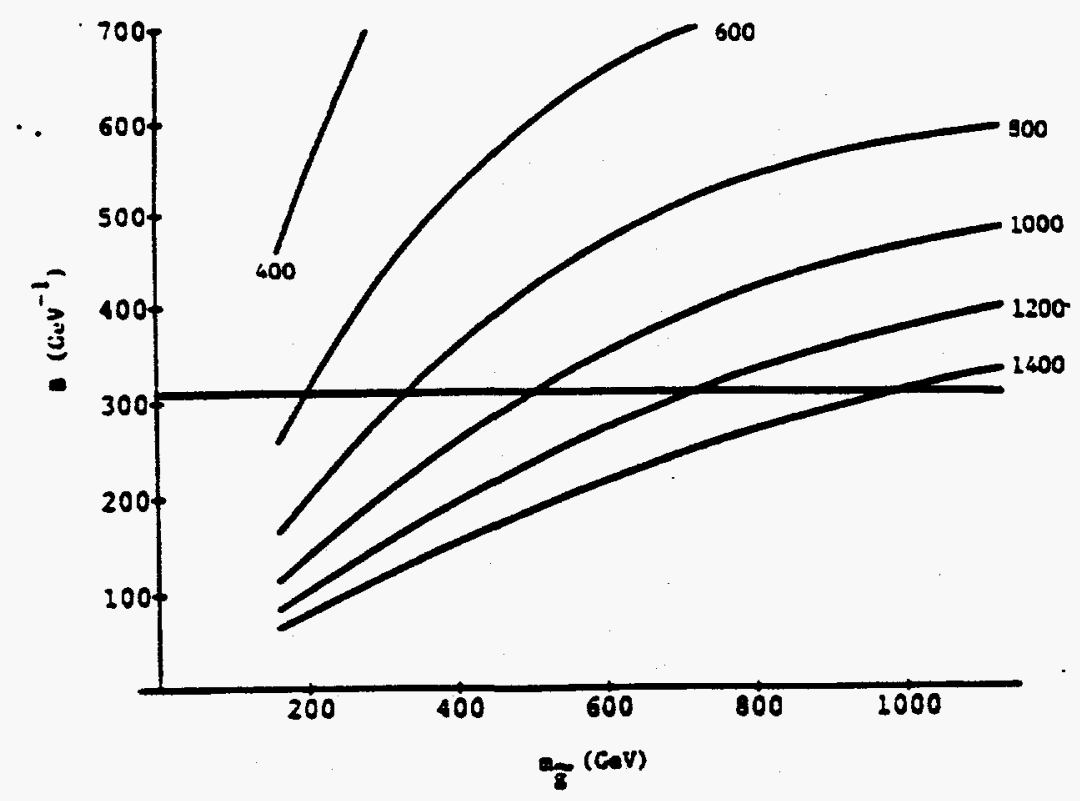

Figure 15: $B$ as a function of $m_{\hat{g}}$ for $m_{t}=125 \mathrm{GeV}, A_{t}=-0.6 m_{0}, \tan \beta=1.73, \mu<0$. An upper bound $m_{0}<1 \mathrm{TeV}$ implies a lower bound on $\boldsymbol{m}_{\tilde{j}}$.

$$
\begin{gathered}
m_{\bar{W}_{1}} \simeq \frac{1}{3} m_{\tilde{g}} \text { for } \mu<0 ; m_{\bar{W}_{1}} \simeq \frac{1}{4} m_{\tilde{g}} \text { for } \mu>0 \\
m_{\tilde{W}_{2}} \cong m_{\tilde{Z}_{3}} \cong m_{\bar{Z}_{4}} \gg m_{\bar{Z}_{1}}
\end{gathered}
$$

In addition the Higgs boson masses obey

$$
m_{H}^{0} \cong m_{A} \cong m_{H \pm} \gg m_{h}
$$

Eqs. (159) and (162) are illustrated in the example of Fig. 7.

Eqs. (159)-(162) are a consequence of the radiative breaking equations Eqs. (89), which determine the parameter $\mu$ [49]. From Eqs. (123)-(125), one sees that the parameters entering there depend on $m_{0}^{2}, \mu^{2}$ and $m_{g} \cong 3 m_{1 / 2}$. Since the proton decay constraints require that $m_{0}$ be relatively large (or if $m_{0}$ is small, $m_{\tilde{g}}$ is large), the $\mu^{2}$ contribution must cancel these large numbers if the r.h.s. of the first equation in (89) is to add up to $\frac{1}{2} M_{Z}^{2}$. Thus in general, one has $\mu^{2} \gg M_{Z}^{2}, \tilde{m}_{2}^{2}$ (a result that often holds also even in the No-scale model when $m_{0}=0$, since then $m_{1 / 2}$ is often large). Expanding Eq. (126) in this limit gives [50]

$$
m_{\tilde{W}_{1}} \cong \tilde{m}_{2}-\frac{M_{W}^{2} \sin 2 \beta}{\mu}, m_{\tilde{W}_{2}} \cong \mu+\frac{M_{W}^{2}}{\mu}
$$

where the $O(1 / \mu)$ corrections are small. Since $\tilde{m}_{2}=\left(\alpha_{2} / \alpha_{3}\right) m_{\tilde{g}} \cong 0.285 m_{\tilde{g}}$ one sees the origin of Eq. (160) since $m_{\bar{w}_{1}}$ is increased (decreased) a small amount depending on whether $\mu$ is negative (positive). The secular equation (127) has two light roots and two heavy roots in this limit: 


$$
\begin{gathered}
m_{\tilde{Z}_{1}} \cong \tilde{m}_{1}-\frac{M_{Z}^{2} \sin 2 \beta \sin ^{2} \theta_{W}}{\mu} ; m_{\tilde{Z}_{2}} \cong \tilde{m}_{2}-\frac{M_{W} \sin 2 \beta}{\mu} \\
m_{\tilde{Z}_{3}, \tilde{z}_{4}} \cong \mid \mu-\frac{1}{2} \frac{M_{Z}^{2}}{\mu}(1 \pm \sin 2 \beta \mid
\end{gathered}
$$

Since $\tilde{m}_{1} / \tilde{m}_{2}=\alpha_{1} / \alpha_{2} \cong 0.506$, Eqs. (163) and (164) lead to Eq. (159) while from Eq. (163) and (165) one deduces Eq. (161). Finally from Eq. (123),(124) one has $m_{1}^{2}+m_{2}^{2}=2 \mu^{2}+m_{0}^{2}+\cdots \gg M_{2}^{2}$, and expanding the formulae for the Higgs boson masses, Eqs. (128)-(130), one deduces Eq. (162). We note also that Eq. (162) implies that the $h$ boson couplings are close to those of the SM.

The theoretical origin of the upper bound $m_{t} \lesssim 180 \mathrm{GeV}$ (in good agreement with the phenomenological result from data analysis [2]) is due to the left-right mixing in the squark mass matrices. Thus from Eq. (118), as $m_{t}$ increases the lower stop eigenvalue $m_{\tilde{t}_{1}}^{2}$ decreases (since the off diagonal elements increase) until $m_{\tilde{t}_{1}}$ is reduced below $45 \mathrm{GeV}$ (it's current experimental bound) beyond which $m_{t_{1}}^{2}$ is rapidly driven tachyonic. Thus large $m_{t}$ reduces the parameter space by requiring $A_{t}$ and ctn $\beta$ to decrease. But proton decay puts a lower bound on etn $\beta$ (i.e. $\tan \beta \lesssim 8$ ), and so eventually the allowed parameter space is exhausted. This occurs for $m_{t} \simeq 180 \mathrm{GeV}$.

To illustrate the significance of the proton decay constraint, one can examine the class of No-scale models (where $m_{0}=0=A_{0}$ ) which allow proton decay. One finds that present data for proton decay excludes this model except in a small corner of the parameter space, $m_{\tilde{g}} \gtrsim 900 \mathrm{GeV}, \tan \beta \lesssim 2$ [51]. Thus No-scale models that are phenomenologically acceptable must forbid proton decay. The one model that does this in a natural way is the flipped $S U(5) \times U(1)$ model [32], which is phenomenologically acceptable and is discussed below.

Future proton decay experiments will be able to give strong tests for models with proton decay. Super Kamiokande will be able to detect the $p \rightarrow \bar{\nu} K^{+}$mode for a lifetime up to $2 \times 10^{33} \mathrm{yr}$ [52] and ICARUS expects to be able to reach $5 \times 10^{33} \mathrm{yr}$ [53]. To exhibit the reach of these experiments, we maximize the partial lifetime $\tau(p \rightarrow \bar{\nu} K)$ at fixed $m_{0}$ by varying all the other parameters over the entire allowed parameter space. Fig. 16 exhibits this for the case $m_{t}=150 \mathrm{GeV}$ for three characteristic values of $M_{H_{3}}{ }^{\prime}[54]$. One can see that ICARUS can test proton decay for $m_{0} \lesssim 800 \mathrm{GeV}$, and Super Kamiokande for $m_{0} \lesssim 600 \mathrm{GeV}$. Fig. 17 shows a similar plot but with the parameters restricted so that $m_{\tilde{w}_{1}}>100 \mathrm{GeV}$. Here we can see that ICARUS can detect proton decay over the entire allowed parameter space, $m_{0}<1 \mathrm{TeV}$, $M_{H_{3}} / M_{G}<10$ (and Super Kamiokande over the region $m_{0} \lesssim 950 \mathrm{GeV}, M_{H_{3}} / M_{G}<10$ ). Thus if ICARUS does not see proton decay, then $m_{\tilde{w}_{1}}<100 \mathrm{GeV}$, and the $\tilde{W}_{1}$ should be observable at LEP200 (and also possibly at the Tevatron). Thus we have the prediction that for models with proton decay obeying the conditions (i) - (iii) stated at the beginning of this section, either proton decay is observable at future underground experiments, or the $\tilde{W}_{1}$ is observable at LEP2 (or the Tevatron). One may in fact show further for the entire parameter space $m_{0}, m_{\tilde{g}}<1 \mathrm{TeV}, M_{H_{3}} / M_{G}<10$, 


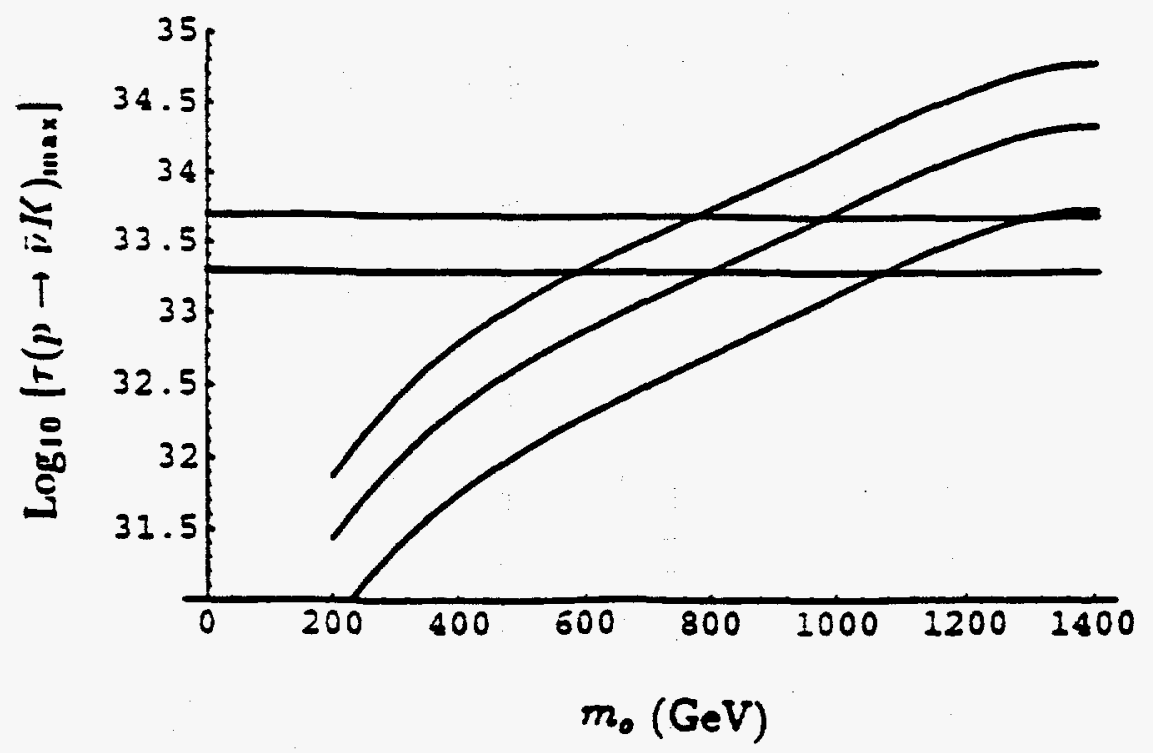

Figure 16: The maximum value of $\tau\left(p \rightarrow \bar{\nu} K^{+}\right)$for $m_{t}=150 \mathrm{GeV}, \mu<0$ as all other parameters are varied. The three curves are for $M_{H_{3}} / M_{G}=3,6,10$ (lower, intermediate, upper curves). ICARUS can detect signals below the upper horizontal line and Super Kamiokande below the lower horizontal line.

that if $\tau(p \rightarrow \overline{\nu K})>1.5 \times 10^{33} \mathrm{yr}$ (which is testable at Super Kamiokande and ICARUS) then either $m_{h}<95 \mathrm{GeV}$ or $m_{\tilde{W}_{1}}<100 \mathrm{GeV}$ i.e. either the light Higgs or the light $\tilde{W}_{1}$ should be observed at LEP200 [54]. Thus models of this type can be experimentally checked in the near future even before the SSC or LHC are operating.

\section{Model Without Proton Decay: Flipped $S U(5) \times U(1)$.}

As discussed in Sec. 14, proton decay is a normal feature of grand unified models and one must do something special in order to suppress it. One natural way of doing this arises in the flipped $S U(5) \times U(1)$ group [32]. Here particles are assigned to the 10 and $\overline{5}$ representations of $S U(5)$ but with $u \leftrightarrow d$ and $e \leftrightarrow \nu$ interchanges. There exists than a $\nu_{R}$ state, and $e_{R}^{c}$ is put into an $S U(5)$ singlet representation. The $p \rightarrow \bar{\nu} K^{+}$amplitude is then suppressed by a factor $O\left(M_{Z} / M_{G}\right)$ and is hence negligible.

One difficulty with this model is that it is not fully unified at the Gut scale as $G$ is still a product group. It has been proposed that unification in this model should actually be at the Planck scale (as one would want in string theory) rather than at the Gut scale [55]. This can be achieved by adding an additional pair of 10 and $\overline{10}$ representations with masses arranged to delay unification until $10^{18} \mathrm{GeV}$. For $\alpha_{3}\left(M_{z}\right)=0.118$ one needs a right $(R)$ handed color triplet and left $(L)$ handed doublet at masses $m_{R}=4.5 \times 10^{6} \mathrm{GeV}$ and $m_{L}=4.1 \times 10^{12} \mathrm{GeV}$. This is illustrated in Fig. 18 [55]. For the strict No-scale model, where $A_{0}=m_{0}=0=B_{0}$, radiative breaking implies a relation between $\tan \beta$ and $m_{\mathfrak{j}}$. One finds that solutions exist for $\mu>0$ only if $m_{t} \lesssim 135 \mathrm{GeV}$, and for $\mu<0$ only if $m_{t} \gtrsim 140 \mathrm{GeV}$. This is illustrated in Fig. 19. 


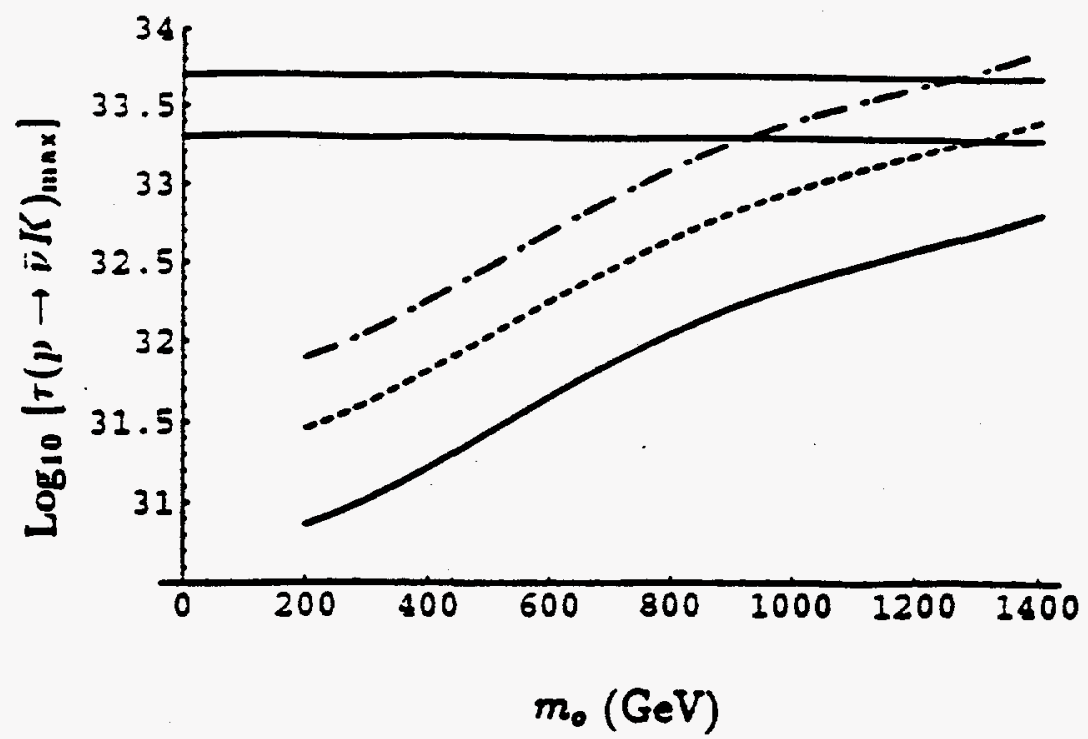

Figure 17: The maximum value of $\tau\left(p \rightarrow \bar{\nu} K^{+}\right)$for $m_{t}=150 \mathrm{GeV}, \mu<0$ subject to $m_{\tilde{W}_{1}}>100 \mathrm{GeV}$. Curves are labeled as in Fig. 14.

Also for $\mu>0$ one has $m_{h}<105 \mathrm{GeV}$, while for $\mu<0$ one has $m_{h}>100 \mathrm{GeV}$. Since the No-scale model has fewer parameters, it can make more precise predictions. Squark, gluino and slepton masses obey the following relations: $m_{\tilde{q}} \approx m_{\tilde{g}}, m_{\tilde{e}_{L}} \approx m_{\tilde{\nu}} \approx 0.3 m_{\tilde{g}}$, $m_{\tilde{e}_{R}} \approx 0.18 m_{\tilde{g}}$. Since the sleptons can be quite light, the tri-lepton SUSY signature $\left[p \bar{p} \rightarrow \tilde{W}_{1}+\tilde{Z}_{2}+X \rightarrow\left(\ell_{1} \bar{\nu}_{1} \tilde{Z}_{1}\right)+\left(\ell_{2} \bar{l}_{2} \tilde{Z}_{1}\right)+X\right]$ should be accessible to the Tevatron [55]. It is interesting that these predictions are quite different from the $S U(5)$ models, and so the two approaches are experimentally distinguishable.

\section{Cosmological Constraints}

The recent COBE data has given strong support to the Big Bang cosmology and to the inflationary scenario. COBE has measured the cosmic microwave background roughly at year 300,000 and finds large scale fluctuations in temperature of size $\Delta T / T \simeq 1^{-5}$. We define the quantity $\Omega=\rho / \rho_{c}$ where $\rho$ is the mass density of the universe and $\rho_{c}$ is the critical mass density need to close the universe, i.e.

$$
\begin{aligned}
\rho_{c} & =3 H_{0}^{2} / 8 \pi G_{N} \\
& =1.88 h_{0}^{2} \times 10^{-20} \mathrm{gm} / \mathrm{cm}^{3}
\end{aligned}
$$

Here $H_{0}$ is the current Hubble constant, $h_{0}$ is the Hubble constant in units of 100 $\mathrm{km} / \mathrm{sec} M p c$, and $G_{N}$ is the Newtonian constant. The inflationary scenario then requires $\Omega=1$.

Recent measurements of $H_{0}$ give a range of

$$
0.65<h_{0}<0.75
$$




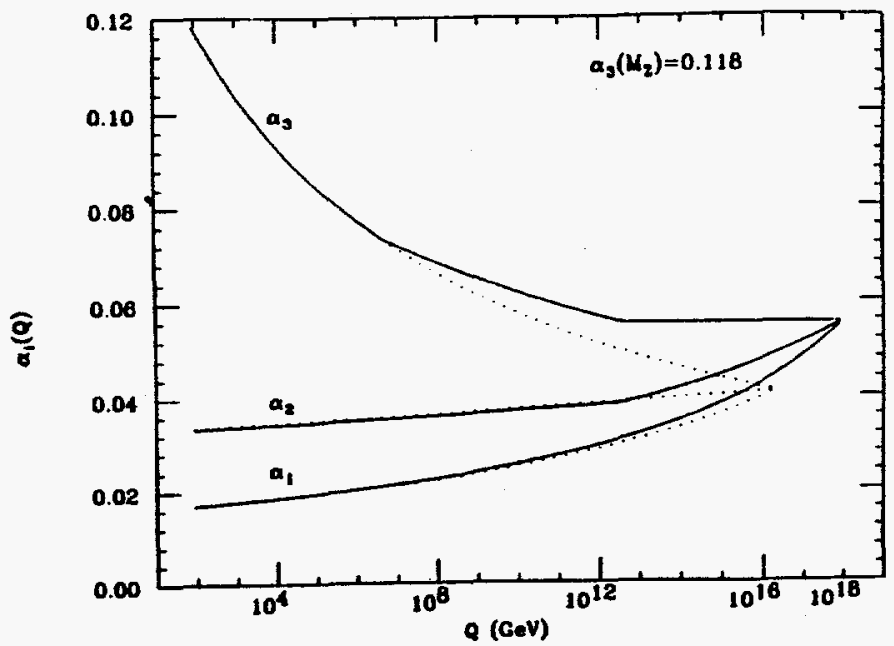

Figure 18: $\alpha_{i}(Q)$ for $\alpha_{3}\left(M_{Z}\right)=0.118$ in the flipped $S U(5) \times U(1)$ model with additional triplet and doublet states. The dotted curves are where unification would be without these additional states.

The visable (baryonic) matter in the universe is estimated at $\Omega_{B} \lesssim 0.1$. Thus there must be a considerable amount of dark matter present in the universe if the inflationary scenario is valid. A popular model is to assume a mix of cold dark matter $(C D M)$ and hot dark matter in the ratio of $\Omega_{C D M} / \Omega_{H D M} \simeq 2$ (i.e. $\Omega_{C D M} \simeq 0.6$, $\left.\Omega_{H D M} \simeq 0.3\right)$. This gives a good fit to the COBE and other cosmological data. [We note one possible problem with this picture having to do with the age of the universe to: For $\Omega=1$, one has $t_{0}=6.5 \times 10^{\circ} \mathrm{yr} / h_{0}$ or $t_{0}=10$ billion years for $h_{0}=0.65$. However, astrophysical calculations of the age of globular clusters gives a $2 \sigma$ spread of ( $13 \lesssim$ to $\lesssim 20$ ) billion years and one would need $h_{0} \lesssim 0.5$ to satisfy this bound.] It is commonly assumed that the HDM are massive $\tau$ neutrinos (left over from the Big Bang). The contribution of massive neutrinos to $\Omega$ is $\Omega_{\nu}=\left(1 / 4 h_{0}^{2}\right)\left(m_{\nu_{\tau}} / 23 \mathrm{eV}\right)$ giving $m_{\nu_{r}} \approx(10) \mathrm{eV}$ for $\Omega_{\nu}=0.3$. Two experiments at CERN, CHORUS and NOMAD, plan to measure $\nu_{\mu}-\nu_{\tau}$ neutrino oscillations and should be able to detect a $\nu_{\tau}$ mass in this range (if the mixing angle $\theta$ obeys $\sin ^{2} 2 \theta \gtrsim 2 \times 10^{-3}$ ).

For SUSY models with $R$ parity, the lightest supersymmetric particle (LSP) is stable. For most models the LSP is the $\tilde{Z}_{1}$. The $\tilde{Z}_{1}$ particles created at the time of the Big Bang are hence a natural candidate for CDM. In general, in order not to overclose the universe, a minimum requirement on models with $R$ parity is

$$
\Omega_{\bar{z}_{1}} h_{0}^{2}<1
$$

If the $\tilde{Z}_{1}$ is the CDM one would need $\Omega_{\bar{z}_{1}} h_{0}^{2} \approx 0.25-0.30$.

While the $\tilde{Z}_{1}$ that were initially created cannot decay, they can annihilate in pairs in the early universe. The main mechanisms are via $h$ and $z$ s-channel poles and squark and slepton $t$-channel poles as shown in Fig. 20. 

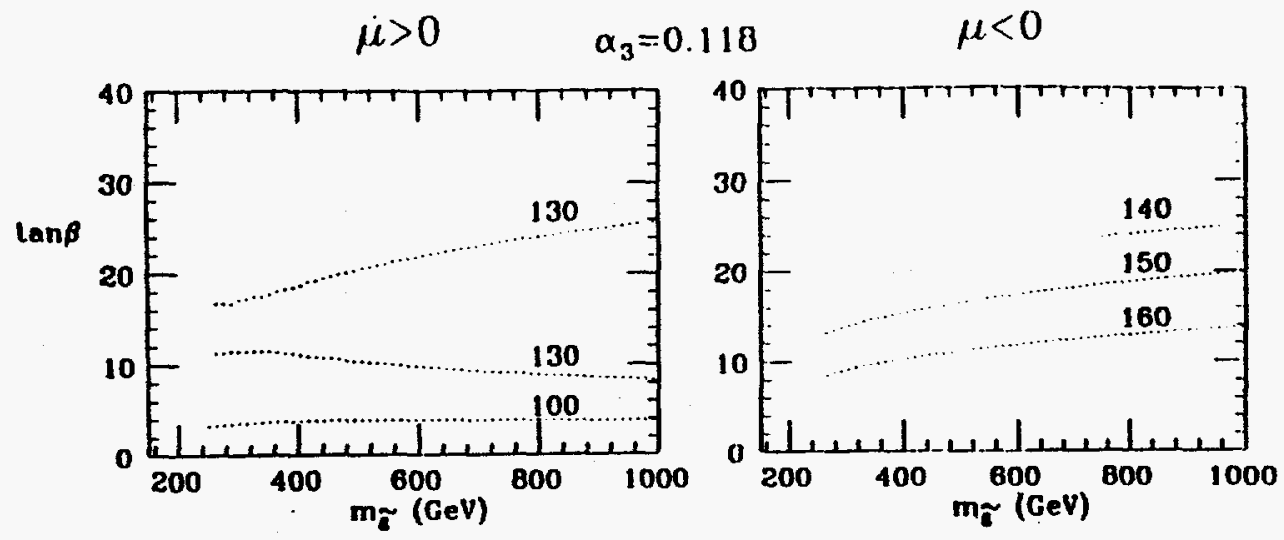

Figure 19: Strict No-scale model with unification arranged to be at $10^{18} \mathrm{GeV}$. Note that for $\mu>0$, the $m_{\bar{g}}-\tan \beta$ relation is double valued with $m_{t} \lesssim 135 \mathrm{GeV}$, while for $\mu<0$, the model predicts $m_{t} \gtrsim 140 \mathrm{GeV}$.

The calculation of $\Omega_{\tilde{z}_{1}} h_{0}^{2}$ follows well known procedures [56] which we briefly summarize now. If $n=$ number/vol of $\tilde{z}_{1}$ 's, then $n$ obeys a Boltzman equation in the early universe:

$$
\frac{d n}{d t}=-3 H n-<\sigma v>\left(n^{2}-n_{0}^{2}\right)
$$

where $H$ is the Hubble constant at time $t, n_{0}$ is the value of $n$ at thermal equilibrium, $\sigma$ is the annihilation cross section, and $v$ is the $\tilde{Z}_{1}$ relative velocity. The first term on the r.h.s. of Eq. (169) represents the decrease in $n$ due to the expansion of the universe (increase in volume). The collision term assumes that the annihilation products go quickly into equilibrium with the thermal background so that the inverse scattering process is governed by $n_{0}$. The expression $<>$ means thermal average, and since annihilation generally occurs at temperatures when the $\tilde{z}_{1}$ are non-relativistic, one may use the Boltzman distribution to calculate it:

$$
\langle\sigma v\rangle=\int_{0}^{\infty} d v v^{2}(\sigma v) e^{-v^{2} / 4 x} / \int_{0}^{\infty} d v v^{2} e^{-v^{2} / 4 x}
$$

where $x \equiv k T / m_{\tilde{Z}_{1}}$ and $T$ is the temperature.

It is convenient to introduce $f(x)=n / T^{3}$, and replace $t$ by $T$ as the independent variable. Then

$$
\frac{d f}{d x}=\frac{m_{\tilde{Z}_{1}}}{k^{3}}\left(\frac{8 \pi^{3} N_{f} G_{N}}{45}\right)^{-\frac{1}{2}}<\sigma v>\left(f^{2}-f_{0}^{2}\right)
$$

where $N_{f}$ is the number of degrees of freedom at temperature $T$ and $f_{0}=n_{0} / T^{3}$ :

$$
f_{0}=\frac{2 k^{3}}{(2 \pi x)^{3 / 2}} e^{-1 / x}
$$

At large $T$, the $\tilde{Z}_{1}$ is in thermal equilibrium with the background and so $f=f_{0}$. However, when the annihilation rate becomes smaller than the expansion rate, the 

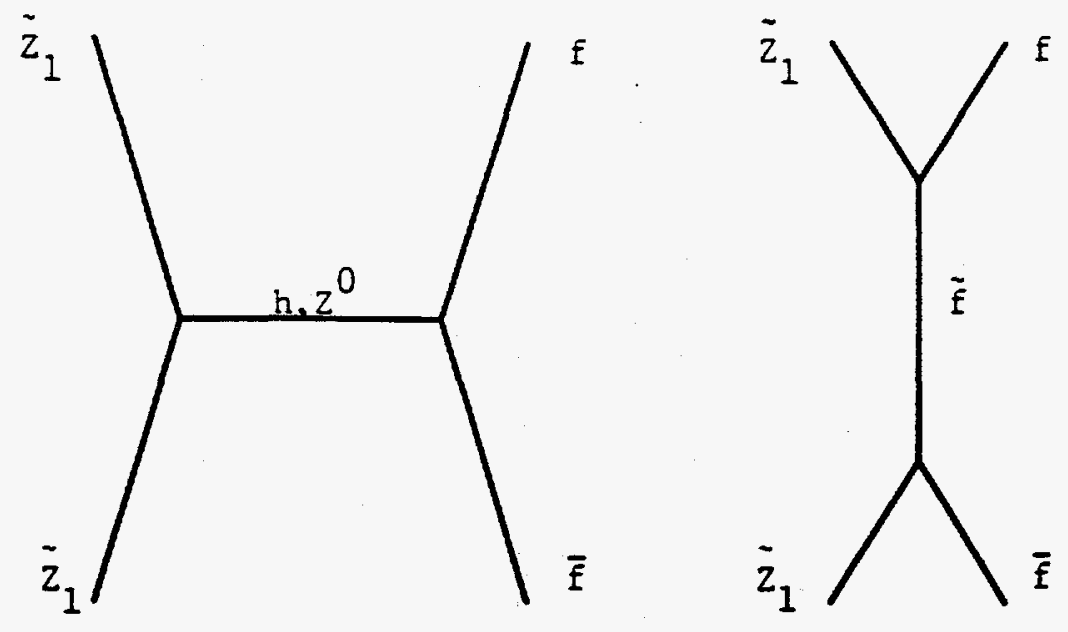

Figure 20: Annihilation processes for $\tilde{Z}_{1}$ pairs. $\tilde{f}=$ squark or slepton and $f=$ quark or lepton.

$\tilde{Z}_{1}$ decouples from the background. This occurs at the "freeze out" temperature $T_{f}$. Then $f$ obeys

$$
\frac{d f}{d x}=\frac{m_{\tilde{Z}_{1}}}{k^{3}}\left(\frac{8 \pi^{3} N_{f} G_{N}}{45}\right)^{-\frac{1}{2}}<\sigma v>f^{2} ; \quad x \leq x_{f} \equiv k T_{f} / m_{\bar{Z}_{1}}
$$

with boundary condition

$$
f\left(x_{f}\right)=f_{0}\left(x_{f}\right)
$$

Eqs. (173),(174) allows one to solve for $x_{f}$ :

$$
x_{f}^{-1} \cong \ln \left[x_{f}^{1 / 2}<\sigma v>_{x_{f}} m_{\bar{z}_{1}} \sqrt{\frac{45}{N_{f} G_{N}}}\right]
$$

In general $x_{f}$ is small, i.e. $x_{f} \approx 1 / 20$ (showing that the freeze out does indeed occur in the non-relativistic domain) and Eq. (175) can be solved for $x_{f}$ by iteration.

Integrating Eq. (173) from $x_{f}$ to $x_{0}=k T_{0} / m_{\tilde{z}_{1}} \cong 0$, where $T_{0}$ is the present temperature, one obtains the relic density at present time $t_{0}$ :

$$
\rho_{\tilde{Z}_{1}}=4.75 \times 10^{-40}\left(\frac{T_{\tilde{Z}_{1}}}{T_{\gamma}}\right)^{3}\left(\frac{T_{\gamma}}{2.75}\right)^{3} \frac{\left(N_{f}\right)^{1 / 2}}{J\left(x_{f}\right)} \mathrm{g} / \mathrm{cm}^{3}
$$

Here $T_{\gamma}$ is the current microwave background temperature, and $J$ is

$$
J\left(x_{f}\right)=\int_{0}^{x_{f}} d x<\sigma v>(x) \mathrm{GeV}^{-2}
$$

From Eq. (166) we have then

$$
\Omega_{\bar{Z}_{1}} h_{0}^{2}=2.53 \times 10^{-11}\left(\frac{T_{\bar{Z}_{1}}}{T_{\gamma}}\right)^{3}\left(\frac{T_{\gamma}}{2.75}\right)^{3} \frac{\left(N_{f}\right)^{1 / 2}}{J\left(x_{f}\right)}
$$


In Eqs. (176) and (178), the factor $\left(T_{\tilde{z}_{1}} / T_{\gamma}\right)^{3}$ arises from the reheating of the photon temperature due to the annihilation of particles of mass less than $k T_{f}[56]$. For a $\tilde{z}_{1}$ with mass of (20-50) $\mathrm{GeV}$, one has [57]

$$
\left(\frac{T_{\gamma}}{T_{\tilde{L}_{1}}}\right)^{3} \cong 18.5 ; \quad N_{f} \cong 289.5
$$

Eqs. (178) and (179) allows an explicit calculation of $\Omega_{\tilde{\bar{Z}}_{1}} h_{0}^{2}$ once the double integral $J\left(x_{f}\right)$ is computed.

We come now to an important fine point. Since $x_{f}$ is small and hence we are in the non-relativistic regime, it had generally been thought previously that one could expand $\sigma v$ in a power series in $v^{2}, \sigma v=a+b v^{2} / 6+\cdots$. Then the thermal average becomes trivial to perform i.e. $\langle\sigma v\rangle \cong a+b x$ (since $\left\langle v^{2}\right\rangle=6 k T / m_{\tilde{Z}_{1}}$ ). However, as has been pointed out [58] this approximation can breakdown badly near a pole or threshold. The breakdown is particularly serious for the physical case at hand due to the narrowness of the $h$ and $z$ poles [59], invalidating a good deal of the earlier analysis. To see what the problem is, consider the $h$-pole where $\sigma v$ has the Breit-Wigner form

$$
\sigma v \cong A_{h} \frac{v^{2}}{\left[\left(v^{2}-\varepsilon_{R}\right)^{2}+\gamma_{R}\right]}
$$

Here $\varepsilon_{R}=\left(m_{h}^{2}-4 m_{\tilde{V}_{1}}^{2}\right) / m_{\tilde{Z}_{1}}^{2}, \gamma_{R}=m_{h} \Gamma_{h} / m_{\tilde{Z}_{1}}^{2}$ and $A_{h}$ is a constant [60]. The $h$ width is $\Gamma_{h} \simeq 2.5 \times 10^{-3} \mathrm{GeV}$ and hence $\gamma_{R}$ is very small. When one thermally averages, one "smears" $v^{2}$, and if $\varepsilon_{R}>0$ (i.e. $2 m_{\tilde{z}_{1}}<m_{h}$ ) then when $v^{2} \approx \varepsilon_{R}$ the integrand becomes very large and one can get a large enhancement in $\langle\sigma v\rangle$. This will modify the value of $x_{f}$ and produce changes in $\Omega_{\bar{z}_{1}} h_{0}^{2}$ by factors as large as 1,000 .

In general one may calculate the integral for $\langle\sigma v\rangle$ numerically without much trouble and thus obtain the correct freeze out parameter $x_{f}$. It is more complicated to calculate the double integral of $J\left(x_{f}\right)$ due to the singular nature of the pole. A convenient procedure is to do the $x$ integral anatylically first to obtain

$$
\begin{aligned}
J_{h} & =\frac{A_{h}}{m_{\bar{Z}_{1}}^{4} 2 \pi^{1 / 2}} \int_{0}^{\infty} d \xi e^{-\xi} \xi^{-\frac{1}{2}}\left\{\frac{1}{2} \ln \left[\frac{\left(4 \xi x_{f}-\varepsilon_{R}\right)^{2}+\gamma_{R}^{2}}{\varepsilon_{R}^{2}+\gamma_{R}^{2}}\right]\right. \\
& \left.+\frac{\varepsilon_{R}}{\gamma_{R}}\left[\tan ^{-1}\left(\frac{4 \xi x_{f}-\varepsilon_{R}}{\gamma_{R}}\right)+\tan ^{-1}\left(\frac{\varepsilon_{R}}{\gamma_{R}}\right)\right]\right\}
\end{aligned}
$$

The remaining integral can then be done numerically without difficulty. A similar analysis can be carried out for the $Z$ pole contributions. For the $t$-channel exchanges of sfermions, the expansion $\sigma v \cong a+b v^{2} / 6$ is good approximation as one is not near a singularity.

Fig. 21 shows the ratio of the approximate value of $\Omega$ to the correct value as a function of $m_{\tilde{g}}$. One sees that the approximate value of $\Omega$ makes a large error in the region prior to each pole. From Fig. 22 this is precisely the region where the correct $\Omega_{\tilde{\mathbf{z}}_{1}} h_{0}^{2}<1$ and hence cosmologically acceptable. One can also see from Fig. 22, that the correct cosmologically allowed region is both larger and in a different $m_{\tilde{g}}$ region 


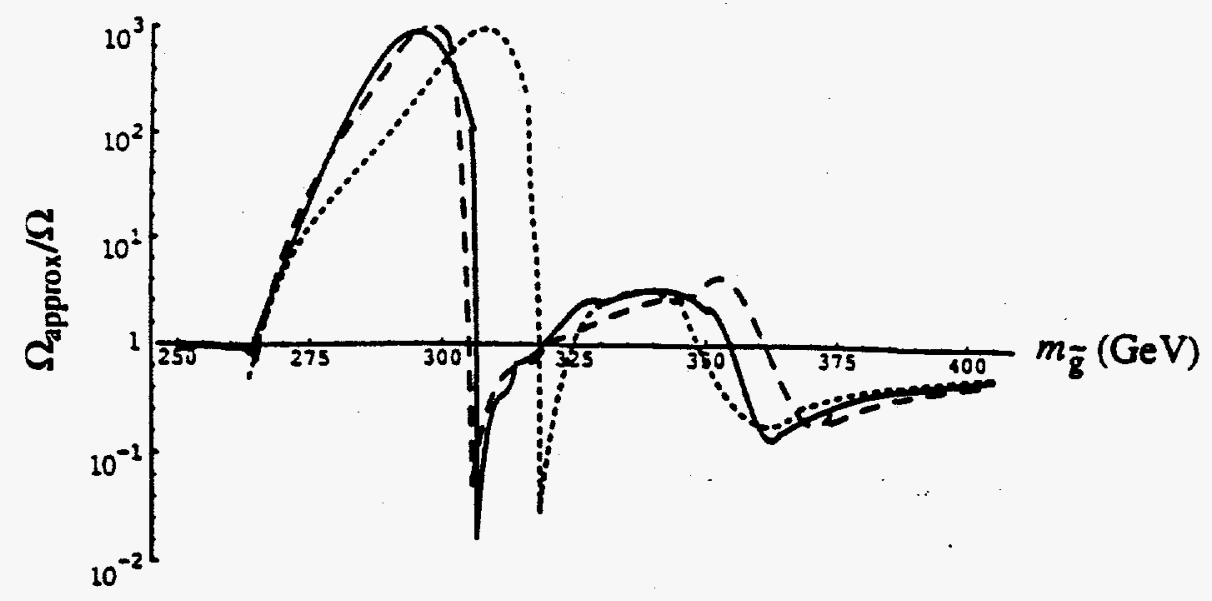

Figure 21: $\Omega_{\text {approx }} / \Omega$ for $m_{0}=700 \mathrm{GeV}, \tan \beta=2.25, \mu>0$ for $m_{t}=110 \mathrm{GeV}, A_{t} / m_{0}=-0.8$ (dashed), $m_{t}=125 \mathrm{GeV}, A_{t} / m_{0}=-0.4$ (solid), and $m_{t}=140 \mathrm{GeV}, A_{t} / m_{0}=0.0$ (dotted). The $h$ and $Z$ poles occur where the curves steeply go from positive to negative.

then for the approximate calculation, showing that the approximate analysis can lead to significant error.

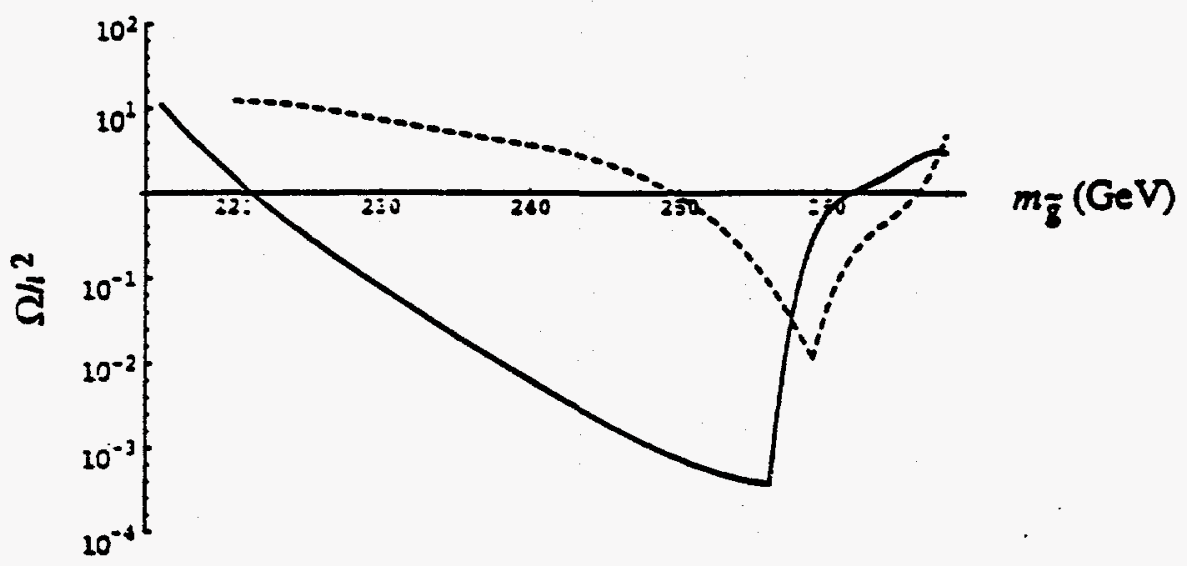

Figure 22: $\Omega h_{0}^{2}$ (solid) and $\left(\Omega h_{0}^{2}\right)_{\text {approx }}$ (dashed) for $m_{t}=125 \mathrm{GeV}, m_{0}=700 \mathrm{GeV}, \tan \beta=1.88$, $A_{i} / m_{0}=0.5, \mu>0$ in the vacinity of the $h$ pole.

We now turn to consider the effect on the parameter space implied by the cosmological constraint of Eq. (168). For the flipped No-scale model $\left(m_{1 / 2} \gg m_{0}\right)$ one generally finds $\Omega h_{0}^{2}<1$, so no additional condition is implied [55]. For models where $m_{0}$ is not zero, one generally finds $\Omega h_{0}^{2}$ to be large, e.g. $O(10)$ except, as illustrated in Figs. 21 and 22 in the region prior to the $h$ and $z$ poles. Thus for the $h$ pole, this would imply an allowed region where $2 m_{\tilde{z}_{1}}<m_{h}$ and since the $\tilde{z}_{1}$ and gluino masses scale, to a band of allowed gluino masses. The cosmological constraint then reduces the five dimensional parameter space $\left(m_{\tilde{g}}, m_{0}, A_{0}, \tan \beta\right.$ and $\left.m_{t}\right)$ to a five 
dimensional shell with thickness in $m_{\tilde{g}}$ of $\approx(5-125) \mathrm{GeV}$ thick. This is illustrated in Figs. 23 and 24. In Fig. 23, the allowed region between the two solid lines comes from the region where $2 m_{\tilde{z}_{1}}<m_{h}$ while the region between the dashed curves comes from the $Z$ pole contribution. Sometimes, these two regions overlap, leading to one large allowed region. An example of this is illustrated in Fig. 24.

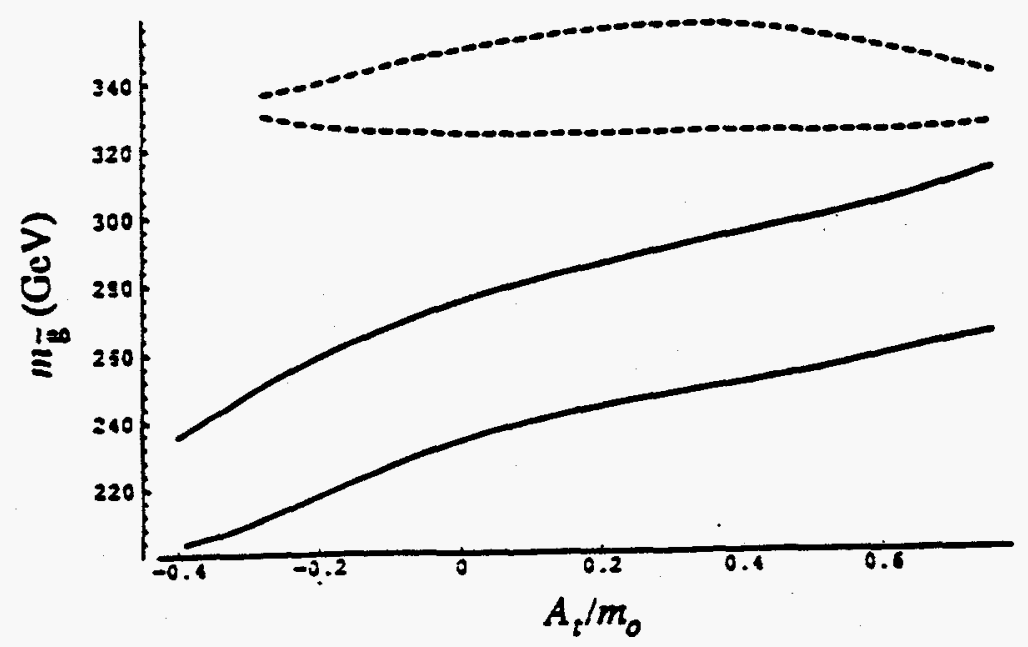

Figure 23: Allowed $m_{\tilde{g}}$ region as a function of $A_{t}$ for $m_{t}=125 \mathrm{GeV}, m_{0}=600 \mathrm{GeV}, \tan \beta=1.73$, $\mu>0$. The allowed region is that between the two solid lines (from the $h$ pole) and that between the two dashed lines (from the $Z$ pole).

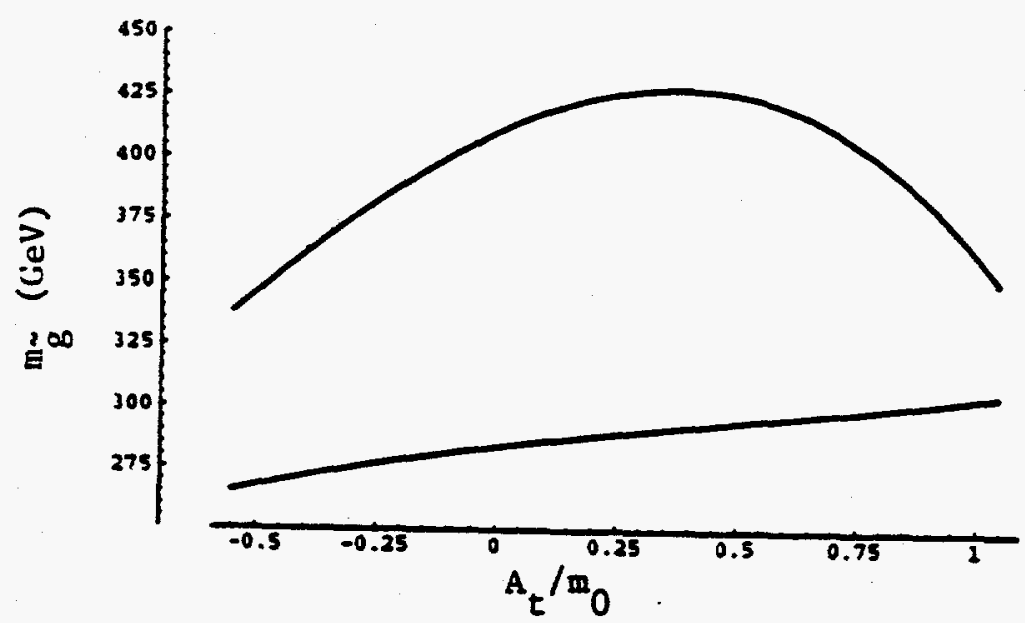

Figure 24: Allowed $m_{\tilde{g}}$ region as a function of $A_{t}$ for $m_{t}=140 \mathrm{GeV}, m_{0}=700, \tan \beta=2.75, \mu>0$.

As a final point we consider the combined relic density constraint Eq. (168) and proton decay constraint. Both of these could operate in a supergravity Gut model with $R$ parity and $S U(5)$-type proton decay. The regions allowed by relic density constraint in Figs. 23 and 24 also obey the current proton decay experimental bounds with $M_{H_{2}} / M_{G}<6\left(B<600 \mathrm{GeV}^{-1}\right)$. Fig. 25 shows the value of the proton decay 
amplitude $B$ for the region allowed by the relic density constraint for $m_{0}=700 \mathrm{GeV}$ as a function of $A_{t}$ (as one varies all other parameters) for $m_{t}=110 \mathrm{GeV}, 125 \mathrm{GeV}$ and $140 \mathrm{GeV}$. Note that the allowed region increases with $m_{t}$. The region below the horizontal line is that allowed by proton decay for $M_{H_{3}} / M_{G}<6$. For $M_{H_{3}} / M_{G}<10$ this line would be at $B=1000 \mathrm{GeV}^{-1}$. The simultaneous relic density and proton decay constraints reduce the allowed parameter space. However, a considerable region is still allowed. For $M_{H_{3}} / M_{G}<6$, one finds that the combined constraints imply $m_{h}<105 \mathrm{GeV}, m_{\tilde{W}_{1}}<100 \mathrm{GeV}, m_{\tilde{Z}_{1}} \lesssim 50 \mathrm{GeV}$ and $m_{t} \lesssim 165 \mathrm{GeV}$. These bounds are relaxed somewhat if one raises $M_{H_{3}}$ to $M_{H_{3}} / M_{G}<10$ (and there are small corners in parameter space where $m_{\tilde{w}_{1}} \approx 300 \mathrm{GeV}$ and $m_{\tilde{z}_{1}} \approx 150 \mathrm{GeV}$ ).

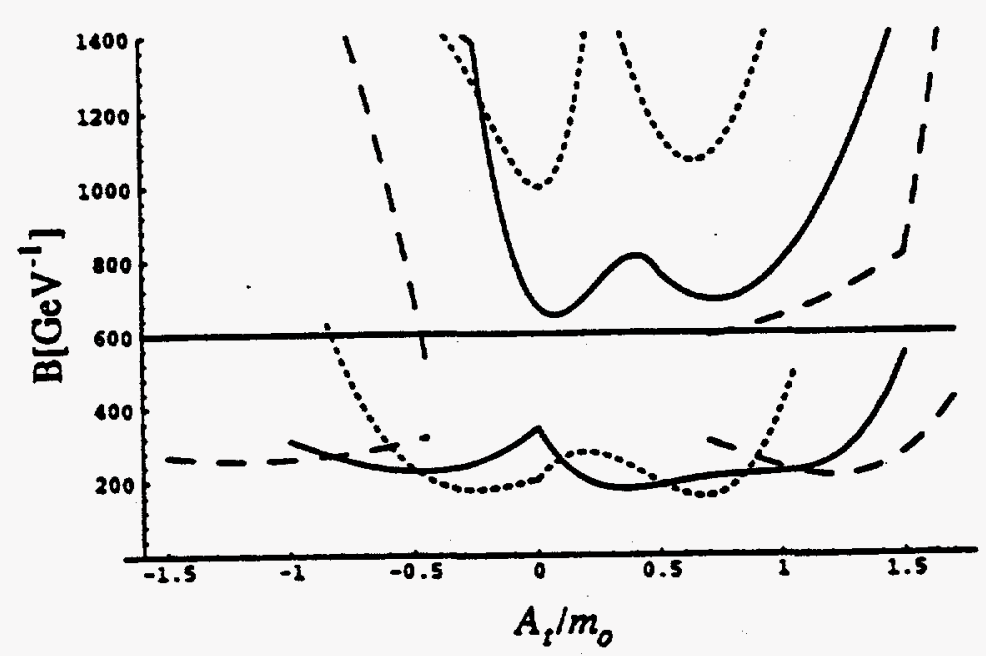

Figure 25: Proton decay amplitude $B$ as a function of $A_{t}$ for $m_{0}=700 \mathrm{GeV}$. The dashed line is for $m_{t}=110 \mathrm{GeV}$, the solid line for $m_{t}=125 \mathrm{GeV}$ and the dotted line for $m_{t}=140 \mathrm{GeV}$. (The gap in the central region for $m_{t}=110 \mathrm{GeV}$ is due to the requirement $m_{h}>60 \mathrm{GeV}$.) The region allowed by the relic density constraint is between the upper and lower curves.

\section{Concluding Remarks}

Several proposals currently exist to remedy the theoretical weaknesses of the Standard Model. Supersymmetry offers a solution to the gauge hierarchy problem in that it offers a natural way for the quadratic divergences of the Higgs self energy to cancel. However, it does this at the expense of greatly enlarging the theoretical structure. Thus the number of particles in the low energy domain is doubled. Further, in order to obtain a phenomenologically acceptable way of spontaneously breaking supersymmetry, one is lead to promoting it to a local gauge symmetry i.e. supergravity. Here, supersymmetry breaking arises from Planck scale interactions, implying that one is dealing with a theory whose natural energy scale is high above the electroweak scale. The recent data, suggesting the validity of supersymmetric grand unification supports this view and implies also the existance of a desert 
between the two scales. Thus the simplest way of consistently implementing the supersymmetric solution to the gauge hierarchy problem is within the framework of supergravity grand unification.

Such an enlargement of the theoretical structure would be unreasonable were it not for two things. First, one can control the unknown nature of the Planck scale interactions which give rise to supersymmetry breaking by imposing simple phenomenological constraints i.e. the absence of flavor changing neutral interactions, and the existance of grand unification. This then allows one to parameterize the supersymmetry breaking sector in terms of only four unknown constants. Hence the masses, production cross sections, decay widths etc. of the 32 new SUSY particles can be obtained in terms of these four new parameters. Second, throughout the entire energy domain, the theory stays within the perturbative domain. As a consequence, explicit calculations of the predictions of the theory can be made and the theoretical extropolations made can be experimentally tested. How to calculate some of the consequences of supergravity grand unified models is given in this report. Some of the more natural models should be tested experimentally in the relatively near future, with detailed tests requiring the SSC or LHC.

There are a number of important questions not explained by supergravity models. Perhaps the most important is the nature of the quark and lepton mass matrices, and this may require Planck scale physics to understand. Superstring theory has the capability of deducing Yukawa couplings, though the huge number of candidate string vacua and the problem of supersymmetry breaking in string theory has inhibited progress in this direction. There have been, however, recent phenomenological analyses of mass matrices [61] based on the idea that the generational hierarchy is scaled by $M_{G} / M_{P \ell}$, implying a Planck physics origin of at least the first two generations mass. Finally we mention the important question of the origin of CP violation. Supergravity models appear to shed no light on this question either. CP violation may also be a Planck physics phenomena (in the Standard Model CP violation is characterized by a phase in the CKM matrix and hence is related to the quark mass matrix). More experimental data is obviously needed to understand this phenomena better.

\section{Acknowledgements}

This work was supported in part by National Science Foundation Grants Nos. PHY-916593 and PHY-9306906. One of us (R.A.) would like to thank the Superconducting Super Collider Laboratory for its kind hospitality during the writing of this report and where part of the work described here was done.

\section{References and Footnotes}

1. G. Altarelli, Les Rencontres de Physique de la Valee D'Aoste, La Thuile, 1993.

2. P. Langacker, Lectures at TASI-92, Boulder, 1992, UPR-O55T, March, 1993. The prediction for $m_{t}$ is $m_{\ell}=150_{-24-20}^{+10+15} \mathrm{GeV}$ where the central value is for the 
Higgs mass $m_{H}=300 \mathrm{GeV}$ and the second error is from assuming $60 \mathrm{GeV}$ $<m_{H}<1000 \mathrm{GeV}$ (smaller $m_{H}$ giving smaller $m_{t}$ ). The best fit occurs for $m_{H}=60 \mathrm{GeV}$ and the upper limit on $m_{t}$ is $m_{t}<208 \mathrm{GeV}$ at 99\% CL (which occurs at $m_{H}=1000 \mathrm{GeV}$ ).

3. P. Langacker, Proc. PASCOS 90-Symposium, Eds. P. Nath and S. Reucroft (World Scientific, Singapore 1990); J. Ellis, S. Kelley and D. V. Nanopoulos, Phys. Lett. 249B, 441 (1990); B260, 131 (1991); U. Amaldi, W. de Boer and H. Furstenau, Phys. Lett. 260B, 447 (1991); F. Anselmo, L. Cifarelli, A. Peterman and A. Zichichi, Nuov. Cim. 104A, 1817 (1991); 115A, 581 (1992).

4. Particle Data Group, Phys. Rev. D45, Part 2 (June, 1992).

5. For a discussion of global supersymmetry see e.g. J. Wess and J. Bagger, Supersymmetry and Supergravity (Princeton University Press, Princeton 1983).

6. Our notation is as follows: diag $\eta_{\mu \nu}=(-1,1,1,1), P^{0}=H=$ Hamiltonian $\left(P_{0}=-P^{0}\right), P^{i}=$ momentum, and $\gamma^{\mu}$ obey $\left\{\gamma^{\mu}, \gamma^{\mu}\right\}=-2 \eta^{\mu \nu}$. In the Majorana representation $\dot{\gamma}^{\mu *}=-\gamma^{\mu}, \tilde{\gamma}^{0}=-\gamma^{0}, \tilde{\gamma}^{i}=+\gamma^{i}$ and $\gamma^{5 *}=-\gamma^{5}, \tilde{\gamma}^{5}=-\gamma^{5}$.

7. R. Haag, J. Lopuszanski and M. Sohnius, Nucl. Phys. B88, 257 (1975).

8. Thus one can consider extended supersymmetries based on $Q_{a}^{A}, A=1 \cdots N$ where $\left\{Q_{\alpha}^{A}, Q_{\beta}^{B \dagger}\right\}=-2\left(P_{L} \gamma^{\mu} \gamma^{0}\right)_{\alpha \beta} \delta^{A B} P_{\mu}$ and add central charges. Algebras with central charges, however, do not allow massless states.

9. S. Weinberg, Phys. Lett. B62, 111 (1976); E. Witten, Nucl. Phys. B202, 253 (1982).

10. M. Grisaru, M. Roĉek and W. Seigel, Nucl. Phys. B159, 429 (1979).

11. Note that by convention, all chiral multiplets are left-handed e.g. $\left(z, \chi_{t}\right)$. Thus instead of using right handed quark or leptons one must use the left-handed conjugate spinors as basic variables e.g. $\left(u^{C}\right)_{L}=\left(u_{R}\right)^{C}$. The corresponding multiplet is denoted by $\left(\tilde{u}^{C}, u_{\tilde{L}}^{C}\right)$. (In the Majorana representation one has $\chi^{C}=$ $\chi^{\dagger}$.)

12. L. Giradello and M. T. Grisaru, Nucl. Phys. B194 65 (1982).

13. In the effective potential, all fields are spin zero bosons, and we will from now on drop the tilde notation for squarks and sleptons.

14. F. Abbe et al., Phys. Rev. Lett. 69, 3439 (1992).

15. M. Davier, Proc. Lepton-Photon High Energy Physics Conference, Geneva, 1991, Eds. S. Hegarty, K. Potter, E. Quercigh (World Scientific, Singapore (1991).

16. S. Franchiotti, B. Kniehl and A. Sirlin, Phys. Rev. D48, 307 (1993).

17. P. Langacker, Ref. [2].

18. H. Bethke, XXVI Proc. Conference on High Energy Physics, Dallas 1992, Ed. J. Sanford, AIP Conf. Proc. No. 272 (1993) G. Altarelli, talk at Europhysics Conference, on High Energy Physics, Marseille, August 1993.

19. M. B. Einhorn and D. R. T. Jones, Nucl. Phys. B196, 475 (1982). 
20. G. Altarelli, Ref. [18].

21. D. Freedman, S. Ferrara and P. van Nieuwenhuizen, Phys. Rev. D13, 3214 (1976); S. Deser and B. Zumino, Phys. Lett. B62, 335 (1976).

22. A. H. Chamseddine, R. Arnowitt and P. Nath, Phys. Rev. Lett. 29, 970 (1982); E. Cremmer, S. Ferrara, L. Girardello and A. van Proeyen, Phys. Lett. 116B, 231 (1982); Nucl. Phys. B212, 413 (1983); E. Witten and J. Bagger, Nucl. Phys. B222, 125 (1983).

23. For a review see $P$. Nath, $R$. Arnowitt and A. H. Chamseddine, "Applied $N=1$ Supergravity" (World Scientific, Singapore (1984); H. P. Nilles, Phys. Rep. 110, 1 (1984).

24. More precisely, the general form of the Kahler potential is $d=z_{i} z_{i}^{\dagger}+f\left(z_{i}\right)+$ $f^{\dagger}\left(z_{i}\right)+\cdots$. One may then remove the function $f\left(z_{i}\right)$ [by a Kahler transformation Eq. (56)] into the superpotential to obtain the form given in text.

25. J. Polonyi, Univ. of Budapest Rep. No. KFKI-1977-93 (1977).

26. H. P. Nilles, Phys. Lett. B115, 193 (1981); S. Ferrara, L. Girardello and H. P. Nilles, Phys. Lett. B125, 457 (1983).

27. A. H. Chamseddine, R. Arnowitt and P. Nath, Phys. Rev. Lett. 49, 970 (1982); L. E. Ibañez, Phys. Lett. B118, 73 (1982); J. Ellis, D. V. Nanopoulos and K. Tamvakis, Phys. Lett. B121, 123 (1983).

28. A full derivation of this result does not exist in the literature. See however, A. H. Chamseddine, R. Arnowitt and P. Nath, Ref. [27]; R. Barbieri, S. Ferrara and C. A. Savoy, Phys. Lett. B119, 343 (1982); L. Hall, J. Lykken and S. Weinberg, Phys. Rev. D27, 2359 (1983); P. Nath, R. Arnowitt and A. H. Chamseddine, Nucl. . Phys. B227, 121 (1983); S. Soni and A. Weldon, Phys. Lett. B126, 215 (1983).

29. E. Witten, Nucl. Phys. B177, 477 (1981); B185, 513 (1981); S. Dimopoulos and H. Georgi, Nucl. Phys. B193, 150 (1981); N. Sakai, Zeit. f. Phys. C11, 153 (1981).

30. B. Grinstein, Nucl. Phys. B206, 387 (1982); H. Georgi, Phys. Lett. B115, 380 (1982).

31. K. Inoue, A. Kakuto and T. Tankano, Prog. Theor. Phys. 75, 664 (1986); A. Anselm and A. Johansen, Phys. Lett. B200, 331 (1988); A. Anselm, Sov. Phys. JETP 67, 663 (1988); R. Barbieri, G. Dvali and A. Strumia, Nucl. Phys. B391, 487 (1993).

32. I. Antoniadis, J. Ellis, J. Hagelin and D. V. Nanopoulos, Phys. Lett. B208, 209 (1988).

33. K. Inoue et al., Prog. Theor. Phys. 68, 927 (1982); L. Ibañez and G. G. Ross, Phys. Lett. B110, 227 (1982); L. Alvarez-Gaumé, J. Polchinski and M. B. Wise, Nucl. Phys. B250, 495 (1983); J. Ellis, J. Hagelin, D. V. Nanopoulos and K. Tamvakis, Phys. Lett. B125, 2275 (1983); L. E. Ibañez and C. Lopez, Phys. Lett. B128, 54 (1983); Nucl. Phys. B233, 545 (1984), L. E. Ibañez, C. 
Lopez and C. Muños, Nucl. Phys. B256, 218 (1985); J. Ellis and F. Zwirner, Nucl. Phys. B338, 317 (1990).

34. A. H. Chamseddine, R. Arnowitt and P. Nath Ref. [27].

35. S. Coleman and E. Weinberg, Phys. Rev. D7, 1888 (1973); S. Weinberg, D7, 2887 (1973).

36. G. Gamberini, G. Ridolfi and F. Zwirner, Nucl. Phys. B331, 331 (1990).

37. R. Arnowitt and P. Nath, Phys. Rev. D46, 3981 (1992).

38. J. M. Frere, D. Jones and S. Raby, Nucl. Phys. B222, 1 (1983); M. Claudson, L. Hall and I. Hinchliffe, Nucl. Phys. B288, 501 (1983); M. Drees, M. Gluck and K. Brassie, Phys. Lett. B157, 164 (1985).

39. Y. Ikada, M. Yamaguchi and T. Yanagida, Prog. Theor. Phys. 85, 1 (1991); J. Ellis, G. Ridolfi and E. Zwirner, Phys. Lett. B257, 83 (1991); H. E. Haber and R. Hampling, Phys. Rev. Lett. 66, 1815 (1991).

40. J. Ellis, S. Kelley and D. V. Nanopoulos, Nucl. Phys. B373, 55 (1992); Phys. Lett. B287, 95 (1992); F. Anselmo et al. [3].

41. R. Barbieri and L. J. Hall, Phys. Rev. Lett. 68, 752 (1992); A. Faraggi, B. Grinstein and S. Meshkov, Phys. Rev. D47, 5018 (1993); L. Hall and U. Sarid, Phys. Rev. Lett. 70, 2673 (1993).

42. P. Langacker and N. Polonsky, Phys. Rev. D47, 4028 (1993).

43. D. Ring and S. Urano, unpublished (1992).

44. Particle Data Group, Phys. Rev. D45, Part 2 (June 1992).

45. S. Weinberg, Phys. Rev. D26, 287 (1982); N. Sakai and T. Yanagida, Nucl. Phys. B197, 533 (1982); S. Dimopoulos, S. Raby and F. Wilczek, Phys. Lett. B112, 133 (1982); J. Ellis, D. V. Nanopoulos and S. Rudaz, Nucl. Phys. B202, 43 (1982); S. Chadha and M. Daniels, Nucl. Phys. B229, 105 (1983); B. A. Campbell, J. Ellis and D. V. Nanopoulos, Phys. Lett. B141, 224 (1984).

46. R. Arnowitt, A. H. Chamseddine and P. Nath, Phys. Lett. B156, 215 (1985); P. Nath, R. Arnowitt and A. H. Chamseddine, Phys. Rev. D32, 2348 (1985).

47. M. B. Gavela et al., Nucl. Phys. B312, 269 (1989).

48. R. Arnowitt and P. Nath, Phys. Rev. Lett. 69, 725 (1992).

49. P. Nath and R. Arnowitt, Phys. Lett. B289 368 (1992).

50. J. F. Gunion and H. E. Haber, Phys. Rev. D37, 2515 (1988).

51. P. Nath and R. Arnowitt, Phys. Lett. B287, 3282 (1992); K. Inoue, M. Kawasaki, M. Yamaguchi and T. Yanagida, Phys. Rev. D45, 328 (1992).

52. Y. Totsuka, Proc. XXIV Conf. on High Energy Physics, Munich, 1988, Eds. R. Kotthaus and J. H. Kuhn (Springer Verlag, Berlin, Heidelberg, 1989).

53. ICARUS Detector Group, Int. Symposium on Neutrino Astrophysics, Takayama, 1992.

54. R. Arnowitt and P. Nath, CTP-TAMU-32/93-NUB-TH-3066/93-SSCL- 
Preprint-440 (1993).

55. J. L. Lopez, D. V. Nanopoulos and A. Zichichi, CERN-TH 6667 (1993).

56. B. W. Lee and S. Weinberg, Phys. Rev. Lett. 39, 165 (1977); D. A. Dicus, E. Kolb and V. Teplitz, Phys. Rev. Lett. 39, 168 (1977); H. Goldberg, Phys. Rev. Lett. 50, 1419 (1983); J. Ellis, J. S. Hagelin, D. V. Nanopoulos, K. Olive and M. Srednicki, Nucl. Phys. B238, 453 (1984).

57. K. Olive, D. Schramm, and G. Steigmann, Nucl. Phys. B180, 497 (1981).

58. K. Griest and D. Seckel, Phys. Rev. D43, 3191 (1991); P. Gondolo and G. Gelmini, Nucl. Phys. B360, 145 (1991).

59. R. Arnowitt and P. Nath, Phys. Lett. B299, 58 (1993) and Erratum ibid B303, 403 (1993); P. Nath and R. Arnowitt, Phys. Rev. Lett. 70, 3696 (1993).

60. J. Lopez, D. V. Nanopoulos and K. Yuan, Nucl. Phys. B370, 445 (1992); M. Drees and M. M. Nojiri, Phys. Rev. D47, 376 (1993).

61. G. Anderson, S. Dimopoulos, L. Hall and S. Raby, Phys. Rev. D47, 3702 (1993).

\section{DISCLAIMER}

This report was prepared as an account of work sponsored by an agency of the United States employees, makes any warranty, States Government nor any agency thereof, nor any of their bility for the accuracy, completeness, or implied, or assumes any legal liability or responsiprocess disclosed, or represents that its usefulness of any information, apparatus, product, or ence herein to any specific commercial use would not infringe privately owned rights. Refermanufacturer, or otherwise does not product, process, or service by trade name, trademark, mendation, or favoring by the United necessarily constitute or imply its endorsement, recomand opinions of authors expressed States Government or any agency thereof. The views United States Government or any agency thereof. 